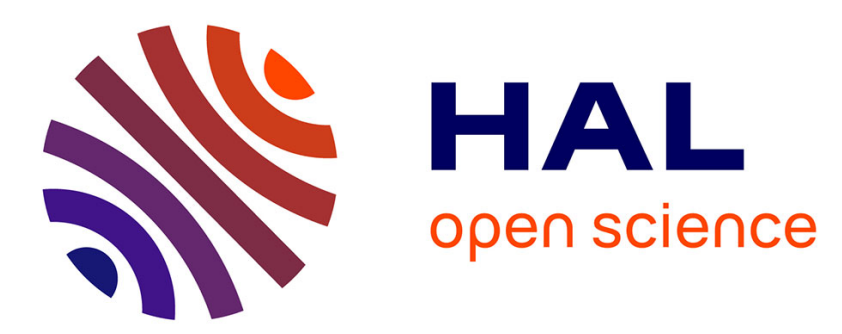

\title{
Convergence Analysis of a Projection Semi-Implicit Coupling Scheme for Fluid-Structure Interaction Problems
}

\author{
Matteo Astorino, Céline Grandmont
}

\section{To cite this version:}

Matteo Astorino, Céline Grandmont. Convergence Analysis of a Projection Semi-Implicit Coupling Scheme for Fluid-Structure Interaction Problems. [Research Report] RR-6996, INRIA. 2009. inria00406493v3

\section{HAL Id: inria-00406493 https://hal.inria.fr/inria-00406493v3}

Submitted on 3 Dec 2010

HAL is a multi-disciplinary open access archive for the deposit and dissemination of scientific research documents, whether they are published or not. The documents may come from teaching and research institutions in France or abroad, or from public or private research centers.
L'archive ouverte pluridisciplinaire $\mathbf{H A L}$, est destinée au dépôt et à la diffusion de documents scientifiques de niveau recherche, publiés ou non, émanant des établissements d'enseignement et de recherche français ou étrangers, des laboratoires publics ou privés. 


\section{N R I A}

INSTITUT NATIONAL DE RECHERCHE EN INFORMATIQUE ET EN AUTOMATIQUE

Convergence Analysis of a Projection Semi-Implicit Coupling Scheme for Fluid-Structure Interaction Problems

Matteo Astorino - Céline Grandmont

$\mathbf{N}^{\circ} 6996$

Juillet 2009

Thème BIO

apport

de recherche 



\title{
Convergence Analysis of a Projection Semi-Implicit Coupling Scheme for Fluid-Structure Interaction Problems
}

\author{
Matteo Astoring* Céline Grandmont $t^{\top *}$ \\ Thème BIO - Systèmes biologiques \\ Équipe-Projet Reo
}

Rapport de recherche $\mathrm{n}^{\circ} 6996$ - Juillet 2009 - 44 pages

\begin{abstract}
In this paper, we provide a convergence analysis of a projection semi-implicit scheme for the simulation of fluid-structure systems involving an incompressible viscous fluid. The error analysis is performed on a fully discretized linear coupled problem: a finite element approximation and a semiimplicit time-stepping strategy are respectively used for space and time discretization. The fluid is described by the Stokes equations, the structure by the classical linear elastodynamics equations and all changes of geometry are neglected. We derive an error estimate in finite time and we prove that the time discretization error for the coupling scheme is at least $\sqrt{\delta t}$. Finally, some numerical experiments that confirm the theoretical analysis are presented.
\end{abstract}

Key-words: fluid-structure interaction, convergence analysis, semi-implicit coupling, Chorin-Temam projection scheme

* INRIA, REO project-team

$\dagger$ Correspondance to: celine.grandmont@inria.fr 


\section{Analyse de convergence d'un schéma de projection semi-implicit pour des problemes d'interaction fluide-structure}

Résumé : Dans cet article on étudie la convergence d'un schéma de projection semi-implicite pour la simulation des phénomènes d'interaction fluide-structure dans le cas où le fluide est visqueux incompressible. L'analyse d'erreur est effectuée sur le systeme linéaire des équations de Stokes couplées aux équations de l'élasticté discretisées en temps et en espace. La discretisation en espace repose sur une approximation éléments finis avec raccordement incompatible à l'interface fluide-structure. La discretisation en temps des équations fluides repose sur le schéma non incrémental de Chorin-Temam et le couplage entre le fluide et la structure se fait de façon semi-implicite en temps. On montre que l'ordre de convergence est au moins de $\sqrt{\delta t}$ en temps alors que l'ordre de convergence en espace dépend de l'opérateur éléments finis de raccord à l'interface et du modèle de structure considéré. Des tests numériques sur la convergence en temps confirment les résultats thériques obtenus.

Mots-clés : interaction fluide-structure, analyse de convergence, couplage semi-implicite, schéma de projection de Chorin-Temam 


\section{Introduction}

The numerical simulation of fluid-structure systems involving an incompressible viscous fluid may be difficult when the so-called added-mass effect is strong [12, 21. This remains true even for really simple linear coupled systems. In literature, a great variety of strategies have been proposed to solve this kind of problem. Generally speaking, they can be classified in three groups of coupling schemes: implicit (also called fully coupled), explicit (or staggered) and semiimplicit. The implicit ones, like for example [3, 19, 23, 36], preserve the energy balance at the interface between fluid and structure but are computationally expensive. Explicit schemes are cheaper but generally unstable in problems with a strong added-mass effect. They require ad hoc treatments of the interface coupling conditions to be stable (see for example [11, 30]). Semi-implicit schemes, which represent a good compromise between the previous two, are cheaper than the implicit ones but more stable than the explicit ones [1, 4, 20, 37. In the latter group, the key idea is to perform an explicit-implicit splitting, based on the use of projection schemes, like the Chorin-Temam method [13, 14, 43] or the algebraic splitting methods 38, 40. At each time step, the projection sub-step is implicitly coupled with the structure, whereas the viscous sub-step, taking into account the convective-viscous effects and the geometrical non-linearities, is treated explicitly.

A number of works has been devoted to the analysis of fluid-structure interaction (FSI) coupling schemes [17, 25, 26, 42, among them we refer in particular to [34, in which the convergence of a fully implicit time dependent linearized FSI problem with non matching finite elements at the interface has been analyzed. There, optimal error estimates are derived.

Our work is devoted to the convergence analysis of the projection semiimplicit algorithm proposed in 20, in which the non-incremental Chorin-Temam projection scheme has been used to obtain a conditionally stable semi-implicit coupling scheme (see [20, Theorem 1]). The analysis will be carried out for a linear fluid-structure problem where the fluid is described by the Stokes equations, the structure by the classical linear elastodynamics equations (linearized elasticity, plate or shell models) and all changes of geometry are neglected. The fluid and structure equations are fully discretized in space and time. In particular, for the coupled problem a space finite element discretization with non-conforming matching at the interface is considered, the fluid is discretized in time with the non-incremental Chorin-Temam projection scheme, while the structure by a first order in time scheme. The coupling conditions at the interface are first order in time approximations and are treated in a semi-implicit way.

The algorithm raises interesting theoretical and numerical problems concerning its accuracy in time and, to our knowledge, this is the first study where error estimates are derived for such schemes. Our aim is to better understand what kind of time accuracy could be expected. It is clear that the scheme will be at most of order one. However, there are two main reasons for which it could be of order less than one. First, it is well-known that the non-incremental ChorinTemam scheme, in a pure hydrodynamic problem, has a reduced time accuracy. For the latter, the following error estimates for the velocities $\boldsymbol{u}_{h}, \tilde{\boldsymbol{u}}_{h}$ and the 
pressure $p_{h}$ were indeed proved (see [2, 18, 28, 29, 39] for more details):

$$
\begin{aligned}
& \left\|\boldsymbol{u}\left(t^{n}\right)-\boldsymbol{u}_{h}^{n}\right\|_{l^{\infty}\left(L^{2}\left(\Omega^{f}\right)\right)}+\left\|\boldsymbol{u}\left(t^{n}\right)-\widetilde{\boldsymbol{u}}_{h}^{n}\right\|_{l^{\infty}\left(L^{2}\left(\Omega^{f}\right)\right)} \leq c\left(h^{k+1}+\delta t\right), \\
& \left\|\boldsymbol{u}\left(t^{n}\right)-\widetilde{\boldsymbol{u}}_{h}^{n}\right\|_{l^{\infty}\left(H^{1}\left(\Omega^{f}\right)\right)}+\left\|p\left(t^{n}\right)-p_{h}^{n}\right\|_{l^{\infty}\left(L^{2}\left(\Omega^{f}\right)\right)} \leq c\left(h^{k}+\sqrt{\delta t}\right),
\end{aligned}
$$

where $k$ is the finite element order (we refer to Section 3 for the notation used). How the accuracy of the fluid scheme acts on the one of the coupled scheme must then be studied. Second, the semi-implicit treatment of the coupling conditions at the fluid-structure interface may also contribute to a modification of the convergence order. Our aim is therefore to investigate through a theoretical analysis and by means of some numerical experiments if and how the convergence rate of the projection semi-implicit algorithm is affected.

The paper is organized as follows. In Section 2, the coupled fluid-structure problem is introduced in a differential form. Initial and boundary conditions are given both for the Stokes and the elastodynamic equations. Subsequently the variational formulation and the corresponding variational setting are presented. Section 3 describes the projection semi-implicit scheme proposed in [20]. Special attention is given to its time and space discretization and to the interface matching operator between the fluid and structure domains. In particular, we remark that for the structure, the Leap-Frog time discretization scheme is used throughout the analysis since a conditional stability has been proved in this case. The study for the Newmark scheme is still an open question from a theoretical point of view, even if computationally it is known to lead to stable algorithms. A non-conforming space discretization of the coupled domain is considered and two possible matching operators are analyzed: a pointwise matching and an integral one. The introduction of a matching operator comes from the fact that, for the time being, the stability analysis of the semi-implicit scheme is proved in [20] under stability conditions that involve different mesh sizes for the fluid and the structure. In Section 4 we build appropriate finite element approximations of the continuous velocity, pressure and displacement. In Section 5, we state the main result and we detail the error analysis of the numerical scheme. A discussion on the optimality in space of the error estimates depending on the structure model, on the interface matching operator and on the polynomial degree of the approximation for the fluid is also presented. Numerical experiments that confirm the theoretical result are given in Section 6. There, the time accuracy of the analyzed semi-implicit coupling scheme is compared to the ones of two other FSI algorithms with respect to an analytical solution. Finally, the last section is devoted to concluding remarks.

\section{Problem setting}

\subsection{Hypotheses and notations}

In order to carry out the analysis we consider a simplified fluid-structure interaction model where a low Reynolds regime is assumed and where the fluidstructure interface undergoes infinitesimal displacements. The fluid might then be simply described by the Stokes equations in a fixed domain $\Omega^{f} \subset \mathbb{R}^{d}, d=2,3$. For the structure we also consider a linear behavior, described either by the classical linearized elastodynamics equations or by equations based on linear 
beam/plate/shell models. The reference domain of the solid is denoted by $\Omega^{s} \subset \mathbb{R}^{d}$. It will be either a domain or a surface of $\mathbb{R}^{d}$ (in this latter case the elastic domain is identified by its mid-surface). We still denote by $\Sigma \stackrel{\text { def }}{=} \partial \Omega^{s} \cap \partial \Omega^{f}$ the fluid-structure interface. When the structure is described by beam/shell model we have $\overline{\Omega^{s}}=\Sigma$, see Figure 1. We indicate by $\Gamma_{f}^{d}\left(\operatorname{resp} . \Gamma_{s}^{d}\right)$ the fluid (resp. structure) boundary which doesn't belong to the fluid-structure interface. Namely $\Gamma_{f}^{d}=\partial \Omega^{f} \backslash \bar{\Sigma}\left(\operatorname{resp} . \Gamma_{s}^{d}=\partial \Omega^{s} \backslash \bar{\Sigma}\right)$.

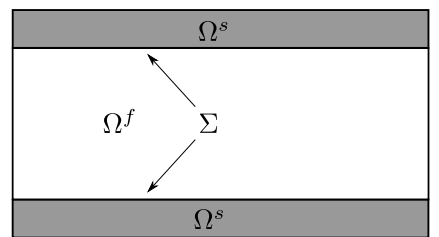

(a) Case $\overline{\Omega^{s}} \neq \Sigma$

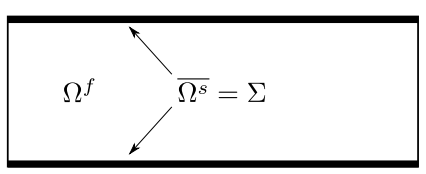

(b) Case $\overline{\Omega^{s}}=\Sigma$

Figure 1: Examples of geometric configurations.

When dealing with a $d$-dimensional structure, our fluid-structure problem reads as follows:

Find the fluid velocity $\boldsymbol{u}: \Omega^{f} \times \mathbb{R}^{+} \rightarrow \mathbb{R}^{d}$, the fluid pressure $p: \Omega^{f} \times \mathbb{R}^{+} \rightarrow \mathbb{R}$ and the structure displacement $\boldsymbol{\eta}: \Omega^{s} \times \mathbb{R}^{+} \rightarrow \mathbb{R}^{d}$ such that

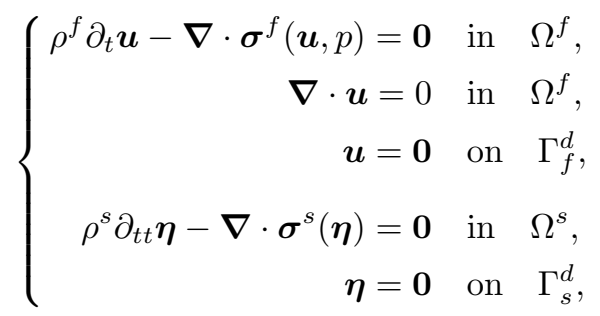

with the interface coupling conditions

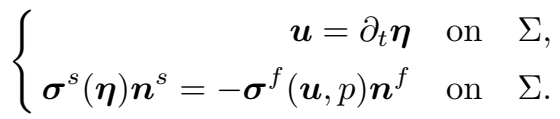

Here, $\rho^{f}$ and $\rho^{s}$ represent respectively the fluid and solid densities, $\boldsymbol{\sigma}^{f}(\boldsymbol{u}, p) \stackrel{\text { def }}{=}$ $-p \boldsymbol{I}+2 \mu \boldsymbol{\epsilon}(\boldsymbol{u})$ stands for the fluid Cauchy stress tensor, with $\mu$ the fluid dynamic viscosity and $\boldsymbol{\epsilon}(\boldsymbol{u}) \stackrel{\text { def }}{=} \frac{1}{2}\left(\boldsymbol{\nabla} \boldsymbol{u}+\boldsymbol{\nabla} \boldsymbol{u}^{\mathrm{T}}\right)$ the strain rate tensor. In the structure equation, $\boldsymbol{\sigma}^{s}(\boldsymbol{\eta})$ stands for the Cauchy stress tensor and is related to the displacement $\boldsymbol{\eta}$ through an appropriate constitutive law (the Hook's law in the case of linearized elasticity). The coupling between the fluid and the structure is realized by imposing at the interface $\Sigma$ the kinematic condition $(3)_{1}$ and the action-reaction principle $(3)_{2}$. In $(3)_{2}, \boldsymbol{n}^{f}$ and $\boldsymbol{n}^{s}$ define, respectively, the outward unit normals to the fluid and solid domains. The coupled problem (2)-(3) is finally completed by the initial conditions

$$
\boldsymbol{u}(0)=\boldsymbol{u}_{0}, \boldsymbol{\eta}(0)=\boldsymbol{\eta}_{0}, \partial_{t} \boldsymbol{\eta}(0)=\boldsymbol{\eta}_{1} .
$$

Before going any further in the analysis, a few remarks are in order: 
- To avoid a pedantic notation the space-time dependence of the unknowns will be occasionally omitted throughout this work, the equivalence of the notations

$$
\boldsymbol{u}=\boldsymbol{u}(t)=\boldsymbol{u}(\boldsymbol{x}, t), \quad p=p(t)=p(\boldsymbol{x}, t) \quad \text { and } \quad \boldsymbol{\eta}=\boldsymbol{\eta}(t)=\boldsymbol{\eta}(\boldsymbol{x}, t),
$$

is however assumed.

- For the sake of simplicity, we choose to consider homogeneous Dirichlet boundary conditions on $\Gamma_{f}^{d}$ and $\Gamma_{s}^{d}$ and no external forces on the coupled system. Nonetheless, the performed convergence analysis could be easily extended to a case where more general assumptions on boundary conditions hold and external forces are considered.

- When the structure motion is described by a beam, shell or membrane model the coupling condition is the kinematic condition (equality of the velocities) and instead of condition $(3)_{2}$, the load applied by the fluid on the elastic media appears in the right-hand side of the structure equation. Nevertheless, the variational formulation of both problems takes the very same form.

\subsection{Variational formulation}

In order to provide a variational setting for the above coupled problem, we consider the usual Sobolev spaces $H^{m}(\Omega), m \geq 0$, with the subspaces

$$
H_{0}^{m}(\Omega) \stackrel{\text { def }}{=}\left\{f \in H^{m}(\Omega): f=0 \quad \text { on } \quad \partial \Omega\right\}
$$

and

$$
H_{0, \Gamma}^{m}(\Omega) \stackrel{\text { def }}{=}\left\{f \in H^{m}(\Omega): f=0 \quad \text { on } \quad \Gamma\right\} .
$$

Here $L^{2}(\Omega)=H^{0}(\Omega)$ and the associated scalar product will be denoted by $(\cdot, \cdot)_{\Omega}$. The space $H^{m}(\Omega)$ is equipped with the norm $\|\cdot\|_{m, \Omega}$ and semi-norm $|\cdot|_{m, \Omega}$ for a given domain $\Omega \subset \mathbb{R}^{d}$. Hence, for the fluid, we define the functional spaces

$$
\begin{gathered}
V^{f} \stackrel{\text { def }}{=}\left\{\boldsymbol{v}^{f} \in H^{1}\left(\Omega^{f}\right): \quad \boldsymbol{v}^{f}=0 \quad \text { on } \quad \Gamma_{f}^{d}\right\}, \\
Q \stackrel{\text { def }}{=}\left\{q \in L^{2}\left(\Omega^{f}\right)\right\},
\end{gathered}
$$

while for the structure, the functional space is denoted by $V^{s} \subseteq\left\{\boldsymbol{v}^{s} \in H^{1}\left(\Omega^{s}\right): \boldsymbol{v}^{s}=0\right.$ on $\left.\Gamma_{s}^{d}\right\}$. In the particular case of linearized elasticity the equality holds, that is

$$
V^{s}=\left\{\boldsymbol{v}^{s} \in H^{1}\left(\Omega^{s}\right): \quad \boldsymbol{v}^{s}=0 \quad \text { on } \quad \Gamma_{s}^{d}\right\} .
$$

We also assume that no distinction will be made between spaces of real-valued functions and spaces of vector-valued functions.

A first weak formulation for the problem (2)-(3) can be derived by multiplying equations $(2)_{1}$ and $(2)_{4}$ by a test function $\boldsymbol{v} \in H_{0}^{1}\left(\Omega^{f} \cup \Omega^{s} \cup \Sigma\right)$ such that $\boldsymbol{v}_{\Omega^{s}} \in V^{s}$. Performing an integration by parts and using the interface condition (3) 2 yields

$$
\begin{aligned}
\rho^{s}\left(\partial_{t t} \boldsymbol{\eta}, \boldsymbol{v}\right)_{\Omega^{s}}+a^{s}(\boldsymbol{\eta}, \boldsymbol{v})+\rho^{f}\left(\partial_{t} \boldsymbol{u}, \boldsymbol{v}\right)_{\Omega^{f}}+2 \mu(\boldsymbol{\epsilon}(\boldsymbol{u}), \boldsymbol{\epsilon}(\boldsymbol{v}))_{\Omega^{f}} & \\
- & (p, \boldsymbol{\nabla} \cdot \boldsymbol{v})_{\Omega^{f}}+(q, \boldsymbol{\nabla} \cdot \boldsymbol{u})_{\Omega^{f}}=0, \\
& \forall(\boldsymbol{v}, q) \in H_{0}^{1}\left(\Omega^{f} \cup \Omega^{s} \cup \Sigma\right) \times L^{2}\left(\Omega^{f}\right), \boldsymbol{v}_{\mid \Omega^{s}} \in V^{s},
\end{aligned}
$$


where $a^{s}(\cdot, \cdot)$ denotes the bilinear form associated with the structure part. We will assume that $a^{s}(\cdot, \cdot)$ defines a scalar product on $V^{s}$ and that exists $r \geq 1$ such that

$$
a^{s}(\boldsymbol{v}, \boldsymbol{v}) \geq C\|\boldsymbol{v}\|_{r, \Omega^{s}}^{2}, \quad \forall \boldsymbol{v} \in V^{s} .
$$

Typically, $r$ will be equal to 1 or 2 . In the case of linearized elasticity, $a^{s}(\cdot, \cdot)$ writes

$$
a^{s}(\boldsymbol{\eta}, \boldsymbol{v})=\left(\boldsymbol{\sigma}^{s}(\boldsymbol{\eta}), \boldsymbol{\epsilon}(\boldsymbol{v})\right)_{\Omega^{s}},
$$

and it is coercive in $H_{0, \Gamma_{s}^{d}}^{1}\left(\Omega^{s}\right)$ (thus $r=1$ ) thanks to Korn's inequality [15].

In (5), a global test function $\boldsymbol{v} \in H_{0}^{1}\left(\Omega^{f} \cup \Omega^{s} \cup \Sigma\right)$ has been used. Nonetheless, we could also have independent test functions that are not necessary equal at the interface $\Sigma$ of the two subdomains. In this case, a more general variational formulation of $(2)-(3)$ could be written by introducing a Lagrange multiplier $\xi$ such that $(\boldsymbol{u}, \boldsymbol{\eta}, p, \xi)$ satisfy

$$
\begin{aligned}
& \rho^{s}\left(\partial_{t t} \boldsymbol{\eta}, \boldsymbol{v}^{s}\right)_{\Omega^{s}}+a^{s}\left(\boldsymbol{\eta}, \boldsymbol{v}^{s}\right)+\rho^{f}\left(\partial_{t} \boldsymbol{u}, \boldsymbol{v}^{f}\right)_{\Omega^{f}}+2 \mu\left(\boldsymbol{\epsilon}(\boldsymbol{u}), \boldsymbol{\epsilon}\left(\boldsymbol{v}^{f}\right)\right)_{\Omega^{f}} \\
&-\left(p, \boldsymbol{\nabla} \cdot \boldsymbol{v}^{f}\right)_{\Omega^{f}}+(q, \boldsymbol{\nabla} \cdot \boldsymbol{u})_{\Omega^{f}}+\left\langle\xi, \boldsymbol{v}^{s}-\boldsymbol{v}^{f}\right\rangle_{\Sigma}=0, \\
& \forall\left(\boldsymbol{v}^{s}, \boldsymbol{v}^{f}, q\right) \in V^{s} \times V^{f} \times Q .
\end{aligned}
$$

Here, the operator $\langle\cdot, \cdot\rangle_{\Sigma}$ denotes the duality product between $\left(H_{00}^{1 / 2}(\Sigma)\right)^{\prime}$ and $H_{00}^{1 / 2}(\Sigma)$. The space $H_{00}^{1 / 2}(\Sigma)$ is a subspace of $H^{1 / 2}(\Sigma)$ with a strictly finer topology, such that, if we extend by zero on $\partial \Omega^{f}$ (resp. $\left.\partial \Omega^{s}\right)$ any $\boldsymbol{v} \in H_{00}^{1 / 2}(\Sigma)$, then the extension belongs to $H^{1 / 2}\left(\partial \Omega^{f}\right)$ (resp. $\left.H^{1 / 2}\left(\partial \Omega^{s}\right)\right)$. For a proof of the existence of the Lagrange multiplier $\xi$ we refer to 34 .

This last formulation will be the one used in our analysis. As a matter of fact, since we will consider non-conforming finite element, the discrete test functions will not be equal at the interface but will only satisfy a weak matching.

Note that any regular enough solution of such a coupled fluid-structure system satisfies an energy equality that writes

$$
\underbrace{\frac{\rho^{f}}{2} \int_{\Omega^{f}}|\boldsymbol{u}|^{2}}+\underbrace{2 \mu \int_{\Omega^{f}}|\boldsymbol{\epsilon}(\boldsymbol{u})|^{2}}+
$$

Fluid kinetic energy Dissipation within the fluid

$$
\underbrace{\frac{\rho^{s}}{2} \int_{\Omega^{s}}\left|\partial_{t} \boldsymbol{\eta}\right|^{2}}+\underbrace{\frac{1}{2} a^{s}(\boldsymbol{\eta}, \boldsymbol{\eta})}
$$

Structure kinetic energy Structure mechanical energy

$$
=\frac{\rho^{f}}{2} \int_{\Omega^{f}}\left|\boldsymbol{u}_{0}\right|^{2}+\frac{\rho^{s}}{2} \int_{\Omega^{s}}\left|\boldsymbol{\eta}_{1}\right|^{2}+\frac{1}{2} a^{s}\left(\boldsymbol{\eta}_{0}, \boldsymbol{\eta}_{0}\right) .
$$

For the well-posedness of the linear fluid-structure interaction problem we refer to [17, 34, 35], and, in all that follows, we will assume that its solution is smooth enough.

Remark 1 We recall that for the coupled problem (2)-(3) different resolution strategies has been developed: from monolithic approaches, to partitioned procedures (see e.g. [22, Chapter 9] for a review). The semi-implicit fluid-structure interaction scheme we will analyze is based on a Dirichlet-Neumann partitioned 
procedure: the fluid problem is solved with a Dirichlet boundary condition at the interface, the structure with a Neumann boundary condition. In this framework, the variational formulation (5) could be equivalently rewritten in the following way:

For all $\left(\boldsymbol{v}^{f}, q\right) \in\left(V^{f} \cap H_{0}^{1}\left(\Omega^{f}\right)\right) \times Q$ and $\boldsymbol{v}^{s} \in V^{s}$, find $(\boldsymbol{u}, p, \boldsymbol{\eta}) \in V^{f} \times Q \times V^{s}$ such that

$$
\left\{\begin{array}{l}
\rho^{f}\left(\partial_{t} \boldsymbol{u}, \boldsymbol{v}^{f}\right)_{\Omega^{f}}+2 \mu\left(\boldsymbol{\epsilon}(\boldsymbol{u}), \boldsymbol{\epsilon}\left(\boldsymbol{v}^{f}\right)\right)_{\Omega^{f}}-\left(p, \boldsymbol{\nabla} \cdot \boldsymbol{v}^{f}\right)_{\Omega^{f}}+(q, \boldsymbol{\nabla} \cdot \boldsymbol{u})_{\Omega^{f}}=0, \\
\boldsymbol{u}=\partial_{t} \boldsymbol{\eta}, \quad \text { on } \quad \Sigma, \\
\rho^{s}\left(\partial_{t t} \boldsymbol{\eta}, \boldsymbol{v}^{s}\right)_{\Omega^{s}}+a^{s}\left(\boldsymbol{\eta}, \boldsymbol{v}^{s}\right)=-\left\langle\mathcal{R}(\boldsymbol{u}, p), \mathcal{L}\left(\boldsymbol{v}^{s}\right)\right\rangle .
\end{array}\right.
$$

The operator $\mathcal{L}$ represents a continuous extension operator from $V^{s}$ into the space $V^{f}$, while $\left\langle\mathcal{R}(\boldsymbol{u}, p), \boldsymbol{v}^{f}\right\rangle$ is defined by

$\left\langle\mathcal{R}(\boldsymbol{u}, p), \boldsymbol{v}^{f}\right\rangle \stackrel{\text { def }}{=} \rho^{f}\left(\partial_{t} \boldsymbol{u}, \boldsymbol{v}^{f}\right)_{\Omega^{f}}+2 \mu\left(\boldsymbol{\epsilon}(\boldsymbol{u}), \boldsymbol{\epsilon}\left(\boldsymbol{v}^{f}\right)\right)_{\Omega^{f}}-\left(p, \boldsymbol{\nabla} \cdot \boldsymbol{v}^{f}\right)_{\Omega^{f}}, \quad \forall \boldsymbol{v}^{f} \in V^{f}$, and it represents the fluid interface load on the structure.

Note that, since $\left\langle\mathcal{R}(\boldsymbol{u}, p), \boldsymbol{v}^{f}\right\rangle$ depends only on the trace of $\boldsymbol{v}^{s}$ at the interface and not on the choice of $\mathcal{L}$, we can easily prove the equivalence between (5) and (8) thanks to the following decomposition

$$
H_{0}^{1}\left(\Omega^{f} \cup \Omega^{s} \cup \Sigma\right)=V_{D} \oplus V_{N},
$$

where

$$
\begin{gathered}
V_{D}=\left\{\boldsymbol{v} \in H_{0}^{1}\left(\Omega^{f} \cup \Omega^{s} \cup \Sigma\right): \boldsymbol{v}_{\mid \Omega^{f}}=\boldsymbol{v}^{f} \in\left(V^{f} \cap H_{0}^{1}\left(\Omega^{f}\right)\right) \quad \text { and } \boldsymbol{v}_{\mid \Omega^{s}}=0\right\}, \\
V_{N}=\left\{\boldsymbol{v} \in H_{0}^{1}\left(\Omega^{f} \cup \Omega^{s} \cup \Sigma\right): \boldsymbol{v}_{\mid \Omega^{s}}=\boldsymbol{v}^{s} \in V^{s} \text { and } \exists \mathcal{L} \text { s.t. } \boldsymbol{v}_{\mid \Omega^{f}}=\mathcal{L}\left(\boldsymbol{v}^{s}\right)\right\} .
\end{gathered}
$$

\section{Semi-implicit projection scheme}

Let us denote by $\delta t>0$ the time step. Assuming that $\tilde{\boldsymbol{u}}^{n}, \boldsymbol{u}^{n}, p^{n}, \boldsymbol{\eta}^{n}$ and $\boldsymbol{\eta}^{n-1}$, approximations of $\boldsymbol{u}\left(t^{n}\right), \boldsymbol{\eta}\left(t^{n}\right), p\left(t^{n}\right)$ and $\boldsymbol{\eta}\left(t^{n-1}\right)$, are known at time $t^{n}=n \delta t$, the semi-implicit projection scheme, introduced in 20, for the coupled system (2)-(3) is given by

Step 1: (explicit viscous-structure coupling)

$$
\left\{\begin{array}{l}
\rho^{f} \frac{\tilde{\boldsymbol{u}}^{n+1}-\boldsymbol{u}^{n}}{\delta t}-2 \mu \operatorname{div}\left(\boldsymbol{\epsilon}\left(\tilde{\boldsymbol{u}}^{n+1}\right)\right)=\mathbf{0}, \text { in } \Omega^{f}, \\
\tilde{\boldsymbol{u}}^{n+1}=\frac{\boldsymbol{\eta}^{n}-\boldsymbol{\eta}^{n-1}}{\delta t}, \text { on } \Sigma
\end{array}\right.
$$

Step 2 : (implicit pressure-structure coupling)

- Fluid projection sub-step:

$$
\left\{\begin{array}{l}
\rho^{f} \frac{\boldsymbol{u}^{n+1}-\tilde{\boldsymbol{u}}^{n+1}}{\delta t}+\nabla p^{n+1}=\mathbf{0}, \quad \text { in } \quad \Omega^{f} \\
\operatorname{div} \boldsymbol{u}^{n+1}=0, \quad \text { in } \quad \Omega^{f}, \\
\boldsymbol{u}^{n+1} \cdot \boldsymbol{n}^{f}=\frac{\boldsymbol{\eta}^{n+1}-\boldsymbol{\eta}^{n}}{\delta t} \cdot \boldsymbol{n}^{f}, \quad \text { on } \Sigma
\end{array}\right.
$$


- Solid sub-step:

$$
\left\{\begin{array}{l}
\rho^{s} \frac{\boldsymbol{\eta}^{n+1}-2 \boldsymbol{\eta}^{n}+\boldsymbol{\eta}^{n-1}}{\delta t^{2}}-\operatorname{div} \boldsymbol{\sigma}^{s}\left(\boldsymbol{\eta}^{n+1}\right)=\mathbf{0}, \text { in } \Omega^{s}, \\
\boldsymbol{\sigma}^{s}\left(\boldsymbol{\eta}^{n+1}\right) \cdot \boldsymbol{n}^{s}=\boldsymbol{\sigma}^{f}\left(\tilde{\boldsymbol{u}}^{n+1}, p^{n+1}\right) \cdot \boldsymbol{n}^{s}, \quad \text { on } \quad \Sigma .
\end{array}\right.
$$

Note that, at the first step, the kinematic condition verified by $\tilde{\boldsymbol{u}}^{n+1}$ is explicit, whereas, for the second step, the kinematic condition verified by $\boldsymbol{u}^{n+1}$ is implicit. The stress applied by the fluid on the structure is thus split in two: the pressure part computed through an implicit procedure and the viscous part computed through an explicit one.

Remark 2 Usually the time discretization for the structure is based on a Newmark scheme. Nevertheless, since the conditional stability of the semi-implicit has been proved only for the Leap-Frog time discretization scheme, we will derive error bounds in this case. The study for the Newmark scheme is still an open question from a theoretical point of view, even if, from a computational point of view, it leads to stable algorithms.

In order to define the variational formulation of $(9)-(11)$ we introduce the spaces

$$
\begin{gathered}
Y^{f} \stackrel{\text { def }}{=}\left\{\boldsymbol{v}^{f} \in L^{2}\left(\Omega^{f}\right): \nabla \cdot \boldsymbol{v}^{f} \in L^{2}\left(\Omega^{f}\right), \boldsymbol{v}^{f} \cdot \boldsymbol{n}=0 \quad \text { on } \Gamma_{f}^{d}\right\}, \\
Y_{0}^{f} \stackrel{\text { def }}{=}\left\{\boldsymbol{v}^{f} \in Y^{f}: \boldsymbol{v}^{f} \cdot \boldsymbol{n}^{f}=0 \quad \text { on } \quad \Sigma\right\} .
\end{gathered}
$$

Assuming that $\tilde{\boldsymbol{u}}^{n}, \boldsymbol{u}^{n}, \boldsymbol{\eta}^{n}$ and $\boldsymbol{\eta}^{n-1}$ are known at time $t^{n}=n \delta t$, the semidiscretized in time formulation writes:

Find $\left(\tilde{\boldsymbol{u}}^{n+1}, \boldsymbol{u}^{n+1}, p^{n+1}, \boldsymbol{\eta}^{n+1}\right) \in V^{f} \times Y^{f} \times L^{2}\left(\Omega^{f}\right) \times V^{s}$ such that:

Step 1: (explicit viscous-structure coupling)

$$
\left\{\begin{array}{l}
\frac{\rho^{f}}{\delta t}\left(\tilde{\boldsymbol{u}}^{n+1}-\boldsymbol{u}^{n}, \tilde{\boldsymbol{v}}^{f}\right)_{\Omega^{f}}+2 \mu\left(\boldsymbol{\epsilon}\left(\tilde{\boldsymbol{u}}^{n+1}\right), \boldsymbol{\epsilon}\left(\tilde{\boldsymbol{v}}^{f}\right)\right)_{\Omega^{f}}=0, \quad \forall \tilde{\boldsymbol{v}}^{f} \in V^{f} \cap H_{0}^{1}\left(\Omega^{f}\right), \\
\tilde{\boldsymbol{u}}^{n+1}=\frac{\boldsymbol{\eta}^{n}-\boldsymbol{\eta}^{n-1}}{\delta t}, \quad \text { on } \Sigma
\end{array}\right.
$$

Step 2: (implicit pressure-structure coupling)

$$
\left\{\begin{array}{l}
\frac{\rho^{f}}{\delta t}\left(\boldsymbol{u}^{n+1}-\tilde{\boldsymbol{u}}^{n+1}, \boldsymbol{v}^{f}\right)_{\Omega^{f}}-\left(p^{n+1}, \boldsymbol{\nabla} \cdot \boldsymbol{v}^{f}\right)_{\Omega^{f}}+\left(q, \boldsymbol{\nabla} \cdot \boldsymbol{u}^{n+1}\right)_{\Omega^{f}}=0, \\
\forall\left(\boldsymbol{v}^{f}, q\right) \in Y_{0}^{f} \times L^{2}\left(\Omega^{f}\right), \\
\boldsymbol{u}^{n+1} \cdot \boldsymbol{n}^{f}=\frac{\boldsymbol{\eta}^{n+1}-\boldsymbol{\eta}^{n}}{\delta t} \cdot \boldsymbol{n}^{f}, \quad \text { on } \Sigma, \\
\frac{\rho^{s}}{\delta t^{2}}\left(\boldsymbol{\eta}^{n+1}-2 \boldsymbol{\eta}^{n}+\boldsymbol{\eta}^{n-1}, \boldsymbol{v}^{s}\right)_{\Omega^{s}}+a^{s}\left(\boldsymbol{\eta}^{n+1}, \boldsymbol{v}^{s}\right)=-\left\langle\mathcal{R}_{\mu}\left(\tilde{\boldsymbol{u}}^{n+1}\right), \mathcal{L}\left(\boldsymbol{v}^{s}\right)\right\rangle \\
-\left\langle\mathcal{R}_{p}\left(\boldsymbol{u}^{n+1}, p^{n+1}\right), \mathcal{L}\left(\boldsymbol{v}^{s}\right)\right\rangle, \quad \forall \boldsymbol{v}^{s} \in V^{s},
\end{array}\right.
$$


where $\mathcal{R}_{\mu}$ and $\mathcal{R}_{p}$ are the fluid residuals

$$
\begin{aligned}
\left\langle\mathcal{R}_{\mu}\left(\tilde{\boldsymbol{u}}^{n+1}\right), \tilde{\boldsymbol{v}}^{f}\right\rangle & \stackrel{\text { def }}{=} \frac{\rho^{f}}{\delta t}\left(\tilde{\boldsymbol{u}}^{n+1}-\boldsymbol{u}^{n}, \tilde{\boldsymbol{v}}^{f}\right)_{\Omega^{f}}+2 \mu\left(\boldsymbol{\epsilon}\left(\tilde{\boldsymbol{u}}^{n+1}\right), \boldsymbol{\epsilon}\left(\tilde{\boldsymbol{v}}^{f}\right)\right)_{\Omega^{f}}, \\
\left\langle\mathcal{R}_{p}\left(\boldsymbol{u}^{n+1}, p^{n+1}\right), \boldsymbol{v}^{f}\right\rangle & \stackrel{\text { def }}{=} \frac{\rho^{f}}{\delta t}\left(\boldsymbol{u}^{n+1}-\tilde{\boldsymbol{u}}^{n+1}, \boldsymbol{v}^{f}\right)_{\Omega^{f}}-\left(p^{n+1}, \boldsymbol{\nabla} \cdot \boldsymbol{v}^{f}\right)_{\Omega^{f}},
\end{aligned}
$$

for all $\tilde{\boldsymbol{v}}^{f}, \boldsymbol{v}^{f} \in V^{f}$. In this scheme the residual $\mathcal{R}$ has been split in two terms, $\mathcal{R}_{\mu}$ and $\mathcal{R}_{p}$, one associated with the viscous step, the other with the projection step.

\subsection{Fully discretized scheme}

In the following, $\left\{\mathcal{T}_{h}^{f}\right\}_{0 \leq h \leq 1}$ (resp. $\left\{\mathcal{T}_{H}^{s}\right\}_{0 \leq H \leq 1}$ ) is the family of triangulations of the domain $\Omega^{f}$ (resp. $\Omega^{s}$ ). For each triangulation the subscripts $h, H \in(0,1]$ refer to the level of refinement of the triangulation. In particular, $h$ is defined as

$$
h \stackrel{\text { def }}{=} \max _{K \in \mathcal{T}_{h}^{f}} h_{K},
$$

with $h_{K}$ the diameter of the element $K$. We define $H$ in an analogous way. In addition, we assume that both families of triangulations are quasi-uniform. For instance, for $\left\{\mathcal{T}_{h}^{f}\right\}_{0<h \leq 1}$, this implies that

$$
\frac{h_{K}}{\rho_{K}}<C_{R}, \quad h_{K} \geq C_{U} h, \quad \forall K \in \mathcal{T}_{h}, \quad \forall h \in(0,1],
$$

where $\rho_{K}$ stands for the diameter of the largest inscribed ball in $K$ and $C_{R}, C_{U}>$ 0 are fixed constants. In the sequel, $C>0$ is used to denote a generic mesh independent constant. We define $Q_{h}$ as an internal Lagrange finite element approximation of $L^{2}\left(\Omega^{f}\right), X_{h}^{f}$ as an internal $\mathcal{C}^{0}$ finite element approximation of $H^{1}\left(\Omega^{f}\right), V_{h}^{f}=X_{h}^{f} \cap V^{f}, V_{H}^{s}$ being an internal, at least $\mathcal{C}^{0}$, finite element space approximation of $V^{s}$. We assume that $X_{h}^{f}$ satisfies:

$$
\left\{\boldsymbol{v}_{h} \in \mathcal{C}^{0}\left(\overline{\Omega^{f}}\right), \boldsymbol{v}_{h \mid K} \in \mathbb{P}_{k}, \forall K \in \mathcal{T}_{h}^{f}\right\} \subset X_{h}^{f}, \text { with } k \geq 1,
$$

where $\mathbb{P}_{k}, k \geq 0$, is the space of polynomials of degree less than or equal to $k$. Moreover, the spaces $V_{h}^{f}$ and $Q_{h}$ are chosen in such a way that every couple $\left(\boldsymbol{v}_{h}, q_{h}\right)$ in $V_{h}^{f} \times Q_{h}$ satisfies the LBB condition [9, 10]. We refer to [17] where, assuming that $\left(V_{h}^{f} \cap H_{0}^{1}\left(\Omega^{f}\right)\right) \times\left(Q_{h} \cap L_{0}^{2}\left(\Omega^{f}\right)\right)$ satisfies the LBB, it is proved that it is also the case for $V_{h}^{f} \times Q_{h}$. Here, the space $L_{0}^{2}\left(\Omega^{f}\right)$ denotes the subspace of $L^{2}\left(\Omega^{f}\right)$ of zero-average functions in $\Omega^{f}$. We also set $V_{h, 0}^{f} \stackrel{\text { def }}{=} V_{h}^{f} \cap H_{0}^{1}\left(\Omega^{f}\right)$. The space $Y^{f}$ (resp. $Y_{0}^{f}$ ) is approximated by $Y_{h}^{f}\left(\right.$ resp. $\left.Y_{h, 0}^{f}\right)$ such that $V_{h}^{f} \subset Y_{h}^{f}$. For a discussion on the possible choices of approximation spaces for the projection method we refer to [28. Furthermore, we denote by $V_{H}^{s}(\Sigma)$ and $V_{h}^{f}(\Sigma)$ the finite element trace spaces associated with $V_{H}^{s}$ and $V_{h}^{f}$, respectively. Note that $V_{H}^{s}(\Sigma)$ is equal to $V_{H}^{s}$ whenever $\Omega^{s} \subset \mathbb{R}^{d-1}$. Finally, $\pi_{h}: V_{H}^{s}(\Sigma) \rightarrow V_{h}^{f}(\Sigma)$ stands for a given interface matching operator. For instance, $\pi_{h}$ can be a linear interpolation operator associated with the fluid finite element (nodal-wise matching) or a projection based operator (see [7). Therefore, the fully discretized problem 
writes as follows:

Find $\left(\tilde{\boldsymbol{u}}_{h}^{n+1}, \boldsymbol{u}_{h}^{n+1}, p_{h}^{n+1}, \boldsymbol{\eta}_{H}^{n+1}\right) \in V_{h}^{f} \times Y_{h}^{f} \times Q_{h} \times V_{H}^{s}$ such that

$$
\begin{aligned}
& \left\{\begin{array}{l}
\frac{\rho^{f}}{\delta t}\left(\tilde{\boldsymbol{u}}_{h}^{n+1}-\boldsymbol{u}_{h}^{n}, \tilde{\boldsymbol{v}}_{h}^{f}\right)_{\Omega^{f}}+2 \mu\left(\boldsymbol{\epsilon}\left(\tilde{\boldsymbol{u}}_{h}^{n+1}\right), \boldsymbol{\epsilon}\left(\tilde{\boldsymbol{v}}_{h}^{f}\right)\right)_{\Omega^{f}}=0, \quad \forall \tilde{\boldsymbol{v}}_{h}^{f} \in V_{h, 0}^{f}, \\
\tilde{\boldsymbol{u}}_{h}^{n+1}=\pi_{h}\left(\frac{\boldsymbol{\eta}_{H}^{n}-\boldsymbol{\eta}_{H}^{n-1}}{\delta t}\right), \quad \text { on } \Sigma,
\end{array}\right. \\
& \left\{\begin{array}{l}
\frac{\rho^{f}}{\delta t}\left(\boldsymbol{u}_{h}^{n+1}-\tilde{\boldsymbol{u}}_{h}^{n+1}, \boldsymbol{v}_{h}^{f}\right)_{\Omega^{f}}-\left(p_{h}^{n+1}, \boldsymbol{\nabla} \cdot \boldsymbol{v}_{h}^{f}\right)_{\Omega^{f}}+\left(q_{h}, \boldsymbol{\nabla} \cdot \boldsymbol{u}_{h}^{n+1}\right)_{\Omega^{f}}=0, \\
\forall\left(\boldsymbol{v}_{h}^{f}, q_{h}\right) \in Y_{h, 0}^{f} \times Q_{h}, \\
\boldsymbol{u}_{h}^{n+1} \cdot \boldsymbol{n}^{f}=\pi_{h}\left(\frac{\boldsymbol{\eta}_{H}^{n+1}-\boldsymbol{\eta}_{H}^{n}}{\delta t}\right) \cdot \boldsymbol{n}^{f}, \quad \text { on } \quad \Sigma, \\
\frac{\rho^{s}}{\delta t^{2}}\left(\boldsymbol{\eta}_{H}^{n+1}-2 \boldsymbol{\eta}_{H}^{n}+\boldsymbol{\eta}_{H}^{n-1}, \boldsymbol{v}_{H}^{s}\right)_{\Omega^{s}}+a^{s}\left(\boldsymbol{\eta}_{H}^{n+1}, \boldsymbol{v}_{H}^{s}\right)=-\left\langle\mathcal{R}_{\mu}\left(\tilde{\boldsymbol{u}}_{h}^{n+1}\right), \mathcal{L}_{h}\left(\boldsymbol{v}_{H}^{s}\right)\right\rangle \\
-\left\langle\mathcal{R}_{p}\left(\boldsymbol{u}_{h}^{n+1}, p_{h}^{n+1}\right), \mathcal{L}_{h}\left(\boldsymbol{v}_{H}^{s}\right)\right\rangle, \quad \forall \boldsymbol{v}_{H}^{s} \in V_{H}^{s} .
\end{array}\right.
\end{aligned}
$$

Here, $\mathcal{L}_{h}: V_{H}^{s}(\Sigma) \longrightarrow V_{h}^{f}$ stands for any given discrete continuous lifting operator satisfying

$$
\mathcal{L}_{h}\left(\boldsymbol{b}_{H}\right)=\pi_{h}\left(\boldsymbol{b}_{H}\right), \quad \text { on } \quad \Sigma .
$$

There are several reasons for introducing non-conforming finite elements at the interface. On the one hand, the fluid-structure problem could involve different type of operators (take, for instance, Stokes coupled with a plate) for which the finite element discretization may lead to non-conforming finite elements at the interface even for matching grids. On the other hand, from a technical point of view, the stability of this semi-implicit scheme is known under sufficient conditions obtained by using different mesh sizes [20. Note, however, that in [1, where the Nitsche's method has been used to impose Dirichlet boundary conditions in the explicit step, this restriction does not appear. We finally recall that for the scheme (16)-(17) the following stability result has been proved in 20]:

Theorem 1 Assume that the interface operator $\pi_{h}: V_{H}^{s}(\Sigma) \longrightarrow V_{h}^{f}(\Sigma)$ is $L^{2}$ stable on $V_{H}^{s}(\Sigma)$. Then, there exists a constant $C>0$, independent of the physical and discretization parameters, such that under the condition

$$
\rho^{s} \geq C\left(\rho^{f} \frac{h}{H^{\alpha}}+\frac{\mu \delta t}{h H^{\alpha}}\right), \quad \text { with } \alpha \stackrel{\text { def }}{=} \begin{cases}0, & \text { if } \overline{\Omega^{s}}=\Sigma, \\ 1, & \text { if } \overline{\Omega^{s}} \neq \Sigma,\end{cases}
$$

the semi-implicit coupling scheme is stable in the energy-norm.

Considering (19), we note that:

- when $\alpha=0$, then the stability condition does not involve the structure mesh size and, in particular, one could set $H=h$, and stabilize the scheme by simply reducing $h$ (and $\delta t$ ).

- when $\alpha=1$, if $\rho^{s}$ is large enough, then one could also consider conforming matching at the interface. Note moreover, that in the numerical simulations performed so far, a conforming matching has always been used without any consequence on stability. 


\subsection{Interface matching operators}

We now give the definitions as well as some properties of two possible matching operators: a pointwise matching and an integral one.

\subsubsection{Pointwise matching}

Let $\pi_{h}: \mathcal{C}^{0}(\bar{\Sigma}) \longrightarrow V_{h}^{f}(\Sigma)$ be the standard finite element interpolation operator associated with the fluid part. As in Theorem 1, we assume that this operator satisfies the assumption:

$$
\pi_{h} \text { is } L^{2} \text {-stable and } H_{0}^{1} \text {-stable in } V_{H}^{s}(\Sigma),
$$

and the following approximation property: $\forall 0 \leq l \leq k+1$, s.t. $V^{s}(\Sigma) \subset H^{l}(\Sigma)$,

$$
\left\|\pi_{h}\left(\boldsymbol{b}_{H}\right)-\boldsymbol{b}_{H}\right\|_{0, \Sigma} \leq C h^{l}\left\|\boldsymbol{b}_{H}\right\|_{l, \Sigma}, \forall \boldsymbol{b}_{H} \in V_{H}^{s}(\Sigma),
$$

where $V^{s}(\Sigma)$ denotes the space of trace functions on the interface $\Sigma$ of $V^{s}$. Note that since we assume that $V^{s}$ is continuously embedded in $H^{r}\left(\Omega^{s}\right)$, then $l \leq r-\frac{\alpha}{2}$ with

$$
\alpha \stackrel{\text { def }}{=} \begin{cases}0, & \text { if } \overline{\Omega^{s}}=\Sigma, \\ 1, & \text { if } \overline{\Omega^{s}} \neq \Sigma .\end{cases}
$$

The property 20 holds true, for instance, when the fluid interface mesh is a sub-triangulation of the solid interface mesh, namely, for all $K \in \mathcal{T}_{h}^{f}$ with $|K \cap \Sigma| \neq 0$ there exists $K^{\prime} \in \mathcal{T}_{H}^{s}$ such that

$$
K \cap \Sigma \subset K^{\prime} \cap \Sigma \text {. }
$$

We refer to 20] for a proof of the $L^{2}$-stability. In a similar way one can prove that the finite element interpolation operator is $H_{0}^{1}$-stable on $V_{H}^{s}(\Sigma)$. Under the same assumption on the interface meshes, estimate (21) can be proved by introducing the Clement operator that is known to verify (21) (see [16]) and by using (20) for the finite element interpolation operator $\pi_{h}$.

Remark 3 Note that if we consider the Clement operator instead of the standard finite element interpolation operator then properties (20) and (21) hold with no additional assumption on the interface mesh (see [16]).

\subsubsection{Mortar matching}

In the case of a mortar matching, the operator $\pi_{h}: L^{2}(\Sigma) \longrightarrow V_{h}^{f}(\Sigma)$ is defined as follows:

$$
\int_{\Sigma}\left(\boldsymbol{b}-\pi_{h}(\boldsymbol{b})\right) \cdot \boldsymbol{\phi}_{h}=0, \forall \boldsymbol{\phi}_{h} \in \tilde{V}_{h}^{f}(\Sigma)
$$

where $\tilde{V}_{h}^{f}(\Sigma)$ is a subspace of $X_{h}^{f}(\Sigma)$ such that

$\left\{\boldsymbol{v}_{h} \in \mathcal{C}^{0}(\bar{\Sigma}), \boldsymbol{v}_{h \mid K} \in \mathbb{P}_{k-1}, \forall K \in \mathcal{T}_{h}^{f}\right.$ with $\left.|K \cap \Sigma| \neq 0\right\} \subset \tilde{V}_{h}^{f}(\Sigma)$, with $k \geq 1$

We will moreover assume that $\tilde{V}_{h}^{f}(\Sigma)$ has good approximation properties. More precisely, let $\tilde{\pi}_{h}$ be the $L^{2}$ orthogonal projection operator on $\tilde{V}_{h}^{f}(\Sigma)$, then $\forall 0 \leq$ $\tau \leq 1, \forall \boldsymbol{v} \in H^{\tau}(\Sigma)$

$$
\left\|\tilde{\pi}_{h}(\boldsymbol{v})-\boldsymbol{v}\right\|_{0, \Sigma}+h^{-\frac{1}{2}}\left\|\tilde{\pi}_{h}(\boldsymbol{v})-\boldsymbol{v}\right\|_{\left(H_{00}^{\frac{1}{2}}(\Sigma)\right)^{\prime}} \leq C h^{\tau}\|\boldsymbol{v}\|_{\tau, \Sigma}
$$


The proof of this property, in the two-dimensional case, can be found in 7,8 . Moreover, we require that the mortar operator $\pi_{h}$ verifies the following stability properties: $\forall \boldsymbol{v} \in H_{0}^{1}(\Sigma)$

$$
\begin{gathered}
\left\|\pi_{h}(\boldsymbol{v})\right\|_{0, \Sigma} \leq C\|\boldsymbol{v}\|_{0, \Sigma}, \\
\left\|\pi_{h}(\boldsymbol{v})\right\|_{H_{0}^{1}(\Sigma)} \leq C\|\boldsymbol{v}\|_{H_{0}^{1}(\Sigma)},
\end{gathered}
$$

and for all $\boldsymbol{v} \in H_{00}^{\frac{1}{2}}(\Sigma)$,

$$
\left\|\pi_{h}(\boldsymbol{v})\right\|_{H_{00}^{\frac{1}{2}}(\Sigma)} \leq C\|\boldsymbol{v}\|_{H_{00}^{\frac{1}{2}}(\Sigma)} .
$$

Finally, the following error estimate is assumed to hold true, $\forall \frac{1}{2} \leq \lambda \leq k+1$

$$
\forall \boldsymbol{v} \in H^{\lambda}(\Sigma) \cap H_{00}^{\frac{1}{2}}(\Sigma), \quad\left\|\pi_{h}(\boldsymbol{v})-\boldsymbol{v}\right\|_{H_{00}^{\frac{1}{2}(\Sigma)}} \leq C h^{\lambda-\frac{1}{2}}\|\boldsymbol{v}\|_{\lambda, \Sigma} .
$$

The proof of 25$]-(28)$ can be found in [5] for the two-dimensional case. Examples of three-dimensional finite element spaces verifying the same inequalities can be found in [6]. Note that these properties imply that $\tilde{V}_{h}^{f}(\Sigma)$ is a good approximation of the space $\left(H_{00}^{\frac{1}{2}}(\Sigma)\right)^{\prime}$.

\section{Construction of the finite element approxima- tions}

In this section, we build an appropriate finite element approximation of the exact solution $(\boldsymbol{u}, p, \boldsymbol{\eta})$ defined in Section 2 , that verifies an implicitly discretized kinematic condition at the interface (see $(29)$ ). A time discretization error will be consequently introduced in the error estimate. In 34, a quite similar construction is made. There, the authors first build approximations of the fluid and structure displacements that match at the interface at each time and then they build velocities satisfying an implicit condition at the interface. Here instead we directly build the approximations of the fluid velocity $\boldsymbol{u}_{h}(t) \in V_{h}^{f}$ and of the structure displacement $\boldsymbol{\eta}_{H}(t) \in V_{H}^{s}$ such that

$$
\boldsymbol{u}_{h}\left(t^{i}\right)=\pi_{h}\left(\frac{\boldsymbol{\eta}_{H}\left(t^{i}\right)-\boldsymbol{\eta}_{H}\left(t^{i-1}\right)}{\delta t}\right), \quad \forall i \text { s.t. } t^{i} \leq T .
$$

Moreover, since we consider two different types of matching operators $\pi_{h}$ at the interface, a discussion on the optimality of the pointwise matching operator, depending on the elastic model, will follow (the mortar operator leading to optimal error estimate in every cases).

First, we define $\left(P_{h}^{f}(\boldsymbol{u})(t), P_{h}^{f}(p)(t), P_{H}^{s}(\boldsymbol{\eta})(t)\right)$ as follows:

$$
\left\{\begin{array}{c}
2 \mu\left(\boldsymbol{\epsilon}\left(P_{h}^{f}(\boldsymbol{u})(t)\right), \boldsymbol{\epsilon}\left(\boldsymbol{v}_{h}\right)\right)_{\Omega^{f}}+\left(P_{h}^{f}(p)(t), \boldsymbol{\nabla} \cdot \boldsymbol{v}_{h}\right)_{\Omega^{f}}= \\
2 \mu\left(\boldsymbol{\epsilon}(\boldsymbol{u})(t), \boldsymbol{\epsilon}\left(\boldsymbol{v}_{h}\right)\right)_{\Omega^{f}}+\left(p(t), \boldsymbol{\nabla} \cdot \boldsymbol{v}_{h}\right)_{\Omega^{f}}, \forall \boldsymbol{v}_{h} \in V_{h}^{f}, \\
\left(q_{h}, \boldsymbol{\nabla} \cdot P_{h}^{f}(\boldsymbol{u})(t)\right)_{\Omega^{f}}=0, \forall q_{h} \in Q_{h},
\end{array}\right.
$$


and

$$
a^{s}\left(P_{H}^{s}(\boldsymbol{\eta})(t), \boldsymbol{v}_{H}\right)=a^{s}\left(\boldsymbol{\eta}(t), \boldsymbol{v}_{H}\right), \forall \boldsymbol{v}_{H} \in V_{H}^{s} .
$$

Then, in a standard way (see [10, 16]), these finite element functions satisfy the following error estimates:

$$
\begin{aligned}
&\left\|P_{h}^{f}(\boldsymbol{u})(t)-\boldsymbol{u}(t)\right\|_{1, \Omega^{f}}+\left\|P_{h}^{f}(p)(t)-p(t)\right\|_{0, \Omega^{f}} \leq \\
& \inf _{\boldsymbol{v}_{h} \in V_{h}^{f}}\left\|\boldsymbol{u}(t)-\boldsymbol{v}_{h}\right\|_{1, \Omega^{f}}+\inf _{q_{h} \in Q_{h}}\left\|p(t)-q_{h}\right\|_{0, \Omega^{f}}, \\
&\left\|P_{H}^{s}(\boldsymbol{\eta})(t)-\boldsymbol{\eta}(t)\right\|_{V^{s}} \leq \inf _{\boldsymbol{v}_{H} \in V_{H}^{s}}\left\|\boldsymbol{\eta}(t)-\boldsymbol{v}_{H}\right\|_{V^{s} .} .
\end{aligned}
$$

We now build corrections of $\left(P_{h}^{f}(\boldsymbol{u}), P_{h}^{s}(\boldsymbol{\eta})\right)$ such that 29$)$ is satisfied and $\left(\boldsymbol{\nabla} \cdot \boldsymbol{u}_{h}, q_{h}\right)_{\Omega^{f}}=0, \forall q_{h} \in Q_{h}$. These two conditions imply that

$$
\int_{\Sigma} \pi_{h}\left(\frac{\boldsymbol{\eta}_{H}\left(t^{i}\right)-\boldsymbol{\eta}_{H}\left(t^{i-1}\right)}{\delta t}\right) \cdot \boldsymbol{n}=0 .
$$

Nevertheless, $\int_{\Sigma} \pi_{h}\left(\frac{P_{H}^{s}(\boldsymbol{\eta})\left(t^{i}\right)-P_{H}^{s}(\boldsymbol{\eta})\left(t^{i-1}\right)}{\delta t}\right) \cdot \boldsymbol{n} \neq 0$. Consequently, we add a correction $\boldsymbol{c}_{H}(t)$ to $P_{H}^{s}(\boldsymbol{\eta})$ so that $\int_{\Sigma} \pi_{h}\left(P_{H}^{s}(\boldsymbol{\eta})(t)+\boldsymbol{c}_{H}(t)\right) \cdot \boldsymbol{n}$ is equal to a given time independent constant. Let $\boldsymbol{u}_{H}^{s} \in V_{H}^{s}$ be an approximation of $\boldsymbol{u}^{s} \in V^{s}$ such that $\int_{\Sigma} \boldsymbol{u}^{s} \cdot \boldsymbol{n} \neq 0$. Then, for $h$ and $H$ sufficiently small $\int_{\Sigma} \pi_{h}\left(\boldsymbol{u}_{H}^{s}\right) \cdot \boldsymbol{n} \neq 0$. We set

$$
\boldsymbol{\eta}_{H}(t)=P_{H}^{s}(\boldsymbol{\eta})(t)+\boldsymbol{c}_{H}(t)=P_{H}^{s}(\boldsymbol{\eta})(t)-\frac{\int_{\Sigma}\left(\pi_{h}\left(P_{H}^{s}(\boldsymbol{\eta})(t)\right)-\boldsymbol{\eta}_{0}\right) \cdot \boldsymbol{n}}{\int_{\Sigma} \pi_{h}\left(\boldsymbol{u}_{H}^{s}\right) \cdot \boldsymbol{n}} \boldsymbol{u}_{H}^{s} .
$$

With this definition $\int_{\Sigma} \pi_{h}\left(\boldsymbol{\eta}_{H}(t)\right) \cdot \boldsymbol{n}=\int_{\Sigma} \boldsymbol{\eta}_{0} \cdot \boldsymbol{n}$, for all $t$. Next, we build a correction of $P_{h}^{f}(\boldsymbol{u})(t)$, denoted $\boldsymbol{z}_{h}(t)$, that is affine in time and such that

$$
P_{h}^{f}(\boldsymbol{u})\left(t^{i}\right)+\boldsymbol{z}_{h}\left(t^{i}\right)=\pi_{h}\left(\frac{\boldsymbol{\eta}_{H}\left(t^{i}\right)-\boldsymbol{\eta}_{H}\left(t^{i-1}\right)}{\delta t}\right) \cdot \boldsymbol{n}, \text { on } \Sigma,
$$

and

$$
\left(\boldsymbol{\nabla} \cdot \boldsymbol{z}_{h}, q_{h}\right)_{\Omega^{f}}=0, \forall q_{h} \in Q_{h} .
$$

This can be done by solving a discrete Stokes problem:

Find $\left(\boldsymbol{z}_{h}\left(t^{i}\right), \mu_{h}\left(t^{i}\right)\right) \in V_{h}^{f} \times\left(Q_{h} \cap L_{0}^{2}\left(\Omega^{f}\right)\right)$ such that

$$
\left\{\begin{array}{llll}
2 \mu\left(\boldsymbol{\epsilon}\left(\boldsymbol{z}_{h}\left(t^{i}\right)\right), \boldsymbol{\epsilon}\left(\boldsymbol{v}_{h}\right)\right)_{\Omega^{f}}+\left(\mu_{h}\left(t^{i}\right), \boldsymbol{\nabla} \cdot \boldsymbol{v}_{h}\right)_{\Omega^{f}} & =0, \quad \forall \boldsymbol{v}_{h} \in V_{h}^{f} \cap H_{0}^{1}\left(\Omega^{f}\right), \\
\left(q_{h}, \boldsymbol{\nabla} \cdot \boldsymbol{z}_{h}\left(t^{i}\right)\right)_{\Omega^{f}} & =0 \quad, \forall q_{h} \in Q_{h} \cap L_{0}^{2}\left(\Omega^{f}\right),
\end{array}\right.
$$

and

$$
\boldsymbol{z}_{h}\left(t^{i}\right)=\pi_{h}\left(\frac{\boldsymbol{\eta}_{H}\left(t^{i}\right)-\boldsymbol{\eta}_{H}\left(t^{i-1}\right)}{\delta t}\right) \cdot \boldsymbol{n}-P_{h}^{f}(\boldsymbol{u})\left(t^{i}\right), \text { on } \Sigma .
$$

This discrete Stokes problem is well-posed and the couple $\left(\boldsymbol{z}_{h}\left(t^{i}\right), \mu_{h}\left(t^{i}\right)\right)$ satisfies

$$
\left\|\boldsymbol{z}_{h}\left(t^{i}\right)\right\|_{1, \Omega^{f}}+\left\|\mu_{h}\left(t^{i}\right)\right\|_{0, \Omega^{f}} \leq C\left\|\pi_{h}\left(\frac{\boldsymbol{\eta}_{H}\left(t^{i}\right)-\boldsymbol{\eta}_{H}\left(t^{i-1}\right)}{\delta t}\right)-P_{h}^{f}(\boldsymbol{u})\left(t^{i}\right)\right\|_{H_{00}^{\frac{1}{2}(\Sigma)}} .
$$


Finally, we define affine in time velocity and pressure such that

$$
\boldsymbol{u}_{h}\left(t^{i}\right)=P_{h}^{f}(\boldsymbol{u})\left(t^{i}\right)+\boldsymbol{z}_{h}\left(t^{i}\right)
$$

and

$$
p_{h}\left(t^{i}\right)=P_{h}^{f}(p)\left(t^{i}\right)+\mu_{h}\left(t^{i}\right) .
$$

We now verify that the considered velocity, pressure and displacement are good approximations of $(\boldsymbol{u}, p, \boldsymbol{\eta})$ in $V^{f} \times Q \times V^{s}$. Thus, the correction terms $\left(\boldsymbol{z}_{h}, \mu_{h}, \boldsymbol{c}_{H}\right)$ have to be estimated. In this study, an error in time as well as an error due to the incompatible matching at the interface will appear. First, we estimate $\boldsymbol{c}_{H}(t)=-\int_{\Sigma}\left(\pi_{h}\left(P_{H}^{s}(\boldsymbol{\eta})(t)\right) \cdot \boldsymbol{n}-\boldsymbol{\eta}_{0} \cdot \boldsymbol{n}\right) \overline{\boldsymbol{u}}_{H}^{s}$, with $\overline{\boldsymbol{u}}_{H}^{s}=\frac{\boldsymbol{u}_{H}^{s}}{\int_{\Sigma} \pi_{h}\left(\boldsymbol{u}_{H}^{s}\right) \cdot \boldsymbol{n}}$

. We note that $\int_{\Sigma} \boldsymbol{\eta}(t) \cdot \boldsymbol{n}=\int_{\Sigma} \boldsymbol{\eta}_{0} \cdot \boldsymbol{n}$ for all $t$, consequently

$$
\boldsymbol{c}_{H}(t)=-\int_{\Sigma}\left(\pi_{h}\left(P_{H}^{s}(\boldsymbol{\eta})(t)\right) \cdot \boldsymbol{n}-\boldsymbol{\eta}(t) \cdot \boldsymbol{n}\right) \overline{\boldsymbol{u}}_{H}^{s} .
$$

Next we distinguish two cases.

Pointwise matching. If the operator $\pi_{h}$ is the interpolation operator then we write

$\boldsymbol{c}_{H}(t)=-\int_{\Sigma}\left(\pi_{h}\left(P_{H}^{s}(\boldsymbol{\eta})(t)\right)-P_{H}^{s}(\boldsymbol{\eta})(t)\right) \cdot \boldsymbol{n} \overline{\boldsymbol{u}}_{H}^{s}+\int_{\Sigma}\left(P_{H}^{s}(\boldsymbol{\eta})(t)-\boldsymbol{\eta}(t)\right) \cdot \boldsymbol{n} \overline{\boldsymbol{u}}_{H}^{s} \cdot$

Consequently, we obtain

$$
\left\|\boldsymbol{c}_{H}(t)\right\|_{V^{s}} \leq C\left(\left\|\pi_{h}\left(P_{H}^{s}(\boldsymbol{\eta})(t)\right)-P_{H}^{s}(\boldsymbol{\eta})(t)\right\|_{0, \Sigma}+\left\|\boldsymbol{\eta}(t)-P_{H}^{s}(\boldsymbol{\eta})(t)\right\|_{0, \Sigma}\right) .
$$

To estimate the first term we use assumption (21), and the second term is estimated using (31). Thus,

$$
\left\|\boldsymbol{c}_{H}(t)\right\|_{V^{s}} \leq C\left(h^{l}\left\|P_{H}^{s}(\boldsymbol{\eta})(t)\right\|_{l, \Sigma}+\inf _{\boldsymbol{v}_{H} \in V_{H}^{s}}\left\|\boldsymbol{\eta}(t)-\boldsymbol{v}_{H}\right\|_{V^{s}}\right)
$$

for all $0 \leq l \leq k+1$ such that $V^{s}(\Sigma)$ is continuously embedded in $H^{l}(\Sigma)$. In particular, $l \leq \min \left(r-\frac{\alpha}{2}, k+1\right)$, where $\alpha$ is defined by 22 and $r$ is such that $V^{s}$ is continuously embedded in $H^{r}\left(\Omega^{s}\right)$. Moreover, due to the definition of $P_{H}^{s}(\boldsymbol{\eta})$, for $l \leq r-\frac{\alpha}{2}$, we have

$$
\left\|P_{H}^{s}(\boldsymbol{\eta})\right\|_{l, \Sigma} \leq C\left\|P_{H}^{s}(\boldsymbol{\eta})\right\|_{r, \Omega^{s}} \leq C\left\|P_{H}^{s}(\boldsymbol{\eta})\right\|_{V^{s}} \leq C\|\boldsymbol{\eta}\|_{V^{s}}
$$

This last inequality yields

$$
\left\|\boldsymbol{c}_{H}(t)\right\|_{V^{s}} \leq C\left(h^{l}\|\boldsymbol{\eta}(t)\|_{V^{s}}+\inf _{\boldsymbol{v}_{H} \in V_{H}^{s}}\left\|\boldsymbol{\eta}(t)-\boldsymbol{v}_{H}\right\|_{V^{s}}\right) .
$$


Mortar matching. Now we consider the case where the matching is ensured by the mortar finite element operator. We have the following decomposition of $c_{H}(t)$

$$
\begin{aligned}
\boldsymbol{c}_{H}(t) & =-\int_{\Sigma}\left(\pi_{h}\left(P_{H}^{s}(\boldsymbol{\eta})(t)\right) \cdot \boldsymbol{n}-\boldsymbol{\eta}(t) \cdot \boldsymbol{n}\right) \overline{\boldsymbol{u}}_{H}^{s} \\
& =-\left(\int_{\Sigma} \pi_{h}\left(P_{H}^{s}(\boldsymbol{\eta})(t)-\boldsymbol{\eta}(t)\right) \cdot \boldsymbol{n}\right) \overline{\boldsymbol{u}}_{H}^{s}+\int_{\Sigma}\left(\pi_{h}(\boldsymbol{\eta}(t)) \cdot \boldsymbol{n}-\boldsymbol{\eta}(t) \cdot \boldsymbol{n}\right) \overline{\boldsymbol{u}}_{H}^{s} .
\end{aligned}
$$

Consequently,

$$
\left\|\boldsymbol{c}_{H}(t)\right\|_{V^{s}} \leq C\left(\left\|\pi_{h}\left(P_{H}^{s}(\boldsymbol{\eta})(t)-\boldsymbol{\eta}(t)\right)\right\|_{0, \Sigma}+\left|\int_{\Sigma}\left(\pi_{h}(\boldsymbol{\eta}(t))-\boldsymbol{\eta}(t)\right) \cdot \boldsymbol{n}\right|\right) .
$$

Since we assume that the integral matching operator is stable in the $L^{2}$-norm, see (25), we obtain

$$
\left\|\boldsymbol{c}_{H}(t)\right\|_{V^{s}} \leq C\left(\left\|P_{H}^{s}(\boldsymbol{\eta})(t)-\boldsymbol{\eta}(t)\right\|_{0, \Sigma}+\left|\int_{\Sigma}\left(\pi_{h}(\boldsymbol{\eta}(t))-\boldsymbol{\eta}(t)\right) \cdot \boldsymbol{n}\right|\right) .
$$

The first term of this inequality is estimated as in 31. For the second term, we take advantage of the definition of the operator to get

$$
\int_{\Sigma}\left(\pi_{h}(\boldsymbol{\eta}(t))-\boldsymbol{\eta}(t)\right) \cdot \boldsymbol{n}=\int_{\Sigma}\left(\pi_{h}(\boldsymbol{\eta}(t))-\boldsymbol{\eta}(t)\right) \cdot\left(\boldsymbol{n}-\tilde{\pi}_{h}(\boldsymbol{n})\right),
$$

and thus

$$
\left|\int_{\Sigma}\left(\pi_{h}(\boldsymbol{\eta}(t))-\boldsymbol{\eta}(t)\right) \cdot \boldsymbol{n}\right| \leq C\left\|\pi_{h}(\boldsymbol{\eta}(t))-\boldsymbol{\eta}(t)\right\|_{H_{00}^{\frac{1}{2}}(\Sigma)}\left\|\boldsymbol{n}-\tilde{\pi}_{h}(\boldsymbol{n})\right\|_{\left(H_{00}^{\frac{1}{2}}(\Sigma)\right)^{\prime}} .
$$

From the assumptions (24) and (28) made respectively on $\tilde{\pi}_{h}$ and $\pi_{h}$ we have

$$
\left|\int_{\Sigma}\left(\pi_{h}(\boldsymbol{\eta}(t))-\boldsymbol{\eta}(t)\right) \cdot \boldsymbol{n}\right| \leq C h^{\lambda}\|\boldsymbol{\eta}(t)\|_{\lambda, \Sigma}, \quad \forall \frac{1}{2} \leq \lambda \leq k+1 .
$$

To summarize, in both cases we have

$$
\left\|\boldsymbol{c}_{H}(t)\right\|_{V^{s}} \leq C\left(h^{l}\|\boldsymbol{\eta}(t)\|_{V^{s}}+\inf _{\boldsymbol{v}_{H} \in V_{H}^{s}}\left\|\boldsymbol{\eta}(t)-\boldsymbol{v}_{H}\right\|_{V^{s}}\right),
$$

where, in case of the finite element interpolation operator,

$$
l \leq \min \left(r-\frac{\alpha}{2}, k+1\right)
$$

while in the mortar case

$$
\frac{1}{2} \leq l \leq k+1
$$

Thus the difference between the continuous displacement $\boldsymbol{\eta}$ and its finite element approximation $\boldsymbol{\eta}_{H}$ in $V^{s}$ can be estimated by the best approximation of $\boldsymbol{\eta}$ in $V_{H}^{s}$ and a term measuring the error due to the non-conforming matching at the interface:

$$
\left\|\boldsymbol{\eta}(t)-\boldsymbol{\eta}_{H}(t)\right\|_{V^{s}} \leq C\left(h^{l}\|\boldsymbol{\eta}(t)\|_{V^{s}}+\inf _{\boldsymbol{v}_{H} \in V_{H}^{s}}\left\|\boldsymbol{\eta}(t)-\boldsymbol{v}_{H}\right\|_{V^{s}}\right),
$$


where $l$ is subject to the same restrictions (34)-(35) as above.

Now we take care of the fluid velocity and estimate $\left\|\boldsymbol{u}\left(t^{i}\right)-\boldsymbol{u}_{h}\left(t^{i}\right)\right\|_{1, \Omega^{f}}$. We have to estimate $\left\|\boldsymbol{z}\left(t^{i}\right)\right\|_{1, \Omega^{f}}$. By definition of $\boldsymbol{z}_{h}$ and remembering (32),

$$
\left\|\boldsymbol{z}_{h}\left(t^{i}\right)\right\|_{1, \Omega^{f}} \leq C\left\|\pi_{h}\left(\frac{\boldsymbol{\eta}_{H}\left(t^{i}\right)-\boldsymbol{\eta}_{H}\left(t^{i-1}\right)}{\delta t}\right)-P_{h}^{f}(\boldsymbol{u})\left(t^{i}\right)\right\|_{H_{00}^{\frac{1}{2}(\Sigma)}} .
$$

But thanks to the assumption of $H_{00}^{1 / 2}$-stability of $\pi_{h}$ (see (20) and (27)) and to the fact that $\pi_{h}\left(\boldsymbol{v}_{h}\right)=\boldsymbol{v}_{h}, \forall \boldsymbol{v}_{h} \in V_{h}^{f}(\Sigma)$

$$
\left\|\boldsymbol{z}_{h}\left(t^{i}\right)\right\|_{1, \Omega^{f}} \leq C\left\|\frac{\boldsymbol{\eta}_{H}\left(t^{i}\right)-\boldsymbol{\eta}_{H}\left(t^{i-1}\right)}{\delta t}-P_{h}^{f}(\boldsymbol{u})\left(t^{i}\right)\right\|_{H_{00}^{\frac{1}{2}}(\Sigma)} .
$$

Note that we have used the $H_{00}^{1 / 2}$-stability of $\pi_{h}$ not only on $V_{H}^{s}(\Sigma)$ but also on the space of functions that writes as a sum of functions of $V_{H}^{s}(\Sigma)$ and of $V_{h}^{f}(\Sigma)$. It is clear the mortar finite element operator verifies this property. For the finite element interpolation operator at the interface it is stable on every spaces whose $\mathcal{C}^{0}$ functions are polynomial on $K \in \mathcal{T}_{h}^{f}$ with $|K \cap \Sigma| \neq 0$. The needed stability property will then be verified for instance when the fluid interface mesh is a sub-triangulation of the solid interface mesh. Consequently

$$
\begin{aligned}
\left\|\boldsymbol{z}_{h}\left(t^{i}\right)\right\|_{1, \Omega^{f}} \leq & C\left(\left\|\frac{\boldsymbol{\eta}_{H}\left(t^{i}\right)-\boldsymbol{\eta}_{H}\left(t^{i-1}\right)}{\delta t}-\partial_{t} \boldsymbol{\eta}_{H}\left(t^{i}\right)\right\|_{H_{00}^{\frac{1}{2}}(\Sigma)}+\left\|\partial_{t} \boldsymbol{\eta}_{H}\left(t^{i}\right)-\partial_{t} \boldsymbol{\eta}\left(t^{i}\right)\right\|_{H_{00}^{\frac{1}{2}}(\Sigma)}\right. \\
& \left.+\left\|\partial_{t} \boldsymbol{\eta}\left(t^{i}\right)-P_{h}^{f}(\boldsymbol{u})\left(t^{i}\right)\right\|_{H_{00}^{\frac{1}{2}}(\Sigma)}\right) \\
\leq & C\left(\left\|\frac{\| \boldsymbol{\eta}_{H}\left(t^{i}\right)-\boldsymbol{\eta}_{H}\left(t^{i-1}\right)}{\delta t}-\partial_{t} \boldsymbol{\eta}_{H}\left(t^{i}\right)\right\|_{H_{00}^{\frac{1}{2}}(\Sigma)}+\left\|P_{H}^{s}\left(\partial_{t} \boldsymbol{\eta}\right)\left(t^{i}\right)-\partial_{t} \boldsymbol{\eta}\left(t^{i}\right)\right\|_{H_{00}^{\frac{1}{2}}(\Sigma)}\right. \\
& \left.+\left\|\partial_{t} \boldsymbol{c}_{H}\left(t^{i}\right)\right\|_{H_{00}^{\frac{1}{2}}(\Sigma)}+\left\|\boldsymbol{u}\left(t^{i}\right)-P_{h}^{f}(\boldsymbol{u})\left(t^{i}\right)\right\|_{H_{00}^{\frac{1}{2}}(\Sigma)}\right) \\
\leq & C\left(\inf _{\boldsymbol{v}_{H} \in V_{H}^{s}}\left\|\partial_{t} \boldsymbol{\eta}\left(t^{i}\right)-\boldsymbol{v}_{H}\right\|_{V^{s}}+\inf _{\boldsymbol{v}_{h} \in V_{h}^{f}}\left\|\boldsymbol{u}\left(t^{i}\right)-\boldsymbol{v}_{h}\right\|_{V^{f}}\right. \\
& \left.+\left\|\frac{\boldsymbol{\eta}_{H}\left(t^{i}\right)-\boldsymbol{\eta}_{H}\left(t^{i-1}\right)}{\delta t}-\partial_{t} \boldsymbol{\eta}_{H}\left(t^{i}\right)\right\|_{H_{00}^{\frac{1}{2}}(\Sigma)}+\left\|\partial_{t} \boldsymbol{c}_{H}\left(t^{i}\right)\right\|_{H_{00}^{\frac{1}{2}}(\Sigma)}\right) .
\end{aligned}
$$

We have then to estimate $\left\|\frac{\| \boldsymbol{\eta}_{H}\left(t^{i}\right)-\boldsymbol{\eta}_{H}\left(t^{i-1}\right)}{\delta t}-\partial_{t} \boldsymbol{\eta}_{H}\left(t^{i}\right)\right\|_{H_{00}^{\frac{1}{2}}(\Sigma)}$ and $\left\|\partial_{t} \boldsymbol{c}_{H}\left(t^{i}\right)\right\|_{H_{00}^{\frac{1}{2}(\Sigma)}}$.

Recalling the definition of $\boldsymbol{\eta}_{H}$, it is easy to see that

$$
\begin{gathered}
\left\|\frac{\boldsymbol{\eta}_{H}\left(t^{i}\right)-\boldsymbol{\eta}_{H}\left(t^{i-1}\right)}{\delta t}-\partial_{t} \boldsymbol{\eta}_{H}\left(t^{i}\right)\right\|_{H_{00}^{\frac{1}{2}}(\Sigma)} \leq\left\|\frac{P_{H}^{s}(\boldsymbol{\eta})\left(t^{i}\right)-P_{H}^{s}(\boldsymbol{\eta})\left(t^{i-1}\right)}{\delta t}-P_{H}^{s}\left(\partial_{t} \boldsymbol{\eta}\right)\left(t^{i}\right)\right\|_{H_{00}^{\frac{1}{2}}(\Sigma)} \\
+\left\|\frac{\boldsymbol{c}_{H}\left(t^{i}\right)-\boldsymbol{c}_{H}\left(t^{i-1}\right)}{\delta t}-\partial_{t} \boldsymbol{c}_{H}\left(t^{i}\right)\right\|_{H_{00}^{\frac{1}{2}}(\Sigma)} . \\
\operatorname{But} \frac{\boldsymbol{c}_{H}\left(t^{i}\right)-\boldsymbol{c}_{H}\left(t^{i-1}\right)}{\delta t}-\partial_{t} \boldsymbol{c}_{H}\left(t^{i}\right)= \\
-\left(\int_{\Sigma} \pi_{h}\left(\frac{P_{H}^{s}(\boldsymbol{\eta})\left(t^{i}\right)-P_{H}^{s}(\boldsymbol{\eta})\left(t^{i-1}\right)}{\delta t}-P_{H}^{s}\left(\partial_{t} \boldsymbol{\eta}\right)\left(t^{i}\right)\right) \cdot \boldsymbol{n}\right) \overline{\boldsymbol{u}}_{H}^{s},
\end{gathered}
$$
which yields

$$
\left\|\frac{\boldsymbol{c}_{H}\left(t^{i}\right)-\boldsymbol{c}_{H}\left(t^{i-1}\right)}{\delta t}-\partial_{t} \boldsymbol{c}_{H}\left(t^{i}\right)\right\|_{H_{00}^{1 / 2}(\Sigma)} \leq C \delta t\|\boldsymbol{\eta}\|_{W^{2, \infty}\left(0, T ; V^{s}\right)},
$$


and thus, due to the definition of $P_{H}^{s}$,

$$
\left\|\frac{\boldsymbol{\eta}_{H}\left(t^{i}\right)-\boldsymbol{\eta}_{H}\left(t^{i-1}\right)}{\delta t}-\partial_{t} \boldsymbol{\eta}_{H}\left(t^{i}\right)\right\|_{H_{00}^{\frac{1}{2}}(\Sigma)} \leq C \delta t\|\boldsymbol{\eta}\|_{W^{2, \infty}\left(0, T ; V^{s}\right)} .
$$

Moreover, as for the estimate of $\left\|\boldsymbol{c}_{H}\right\|_{H_{00}^{\frac{1}{2}}(\Sigma)}$, we have

$$
\left\|\partial_{t} \boldsymbol{c}_{H}\left(t^{i}\right)\right\|_{H_{00}^{\frac{1}{2}(\Sigma)}} \leq C\left\|\partial_{t} \boldsymbol{c}_{H}\left(t^{i}\right)\right\|_{V^{s}} \leq C\left(h^{l}\left\|\partial_{t} \boldsymbol{\eta}\left(t^{i}\right)\right\|_{V^{s}}+\inf _{\boldsymbol{v}_{H} \in V_{H}^{s}}\left\|\partial_{t} \boldsymbol{\eta}\left(t^{i}\right)-\boldsymbol{v}_{H}\right\|_{V^{s}}\right) .
$$

with $l$ verifying either (34) in the interpolation case or 35 in the mortar case. Therefore,

$$
\begin{array}{r}
\left\|\boldsymbol{z}_{h}\left(t^{i}\right)\right\|_{1, \Omega^{f}} \leq C\left(\delta t\|\boldsymbol{\eta}\|_{W^{2, \infty}\left(0, T ; V^{s}\right)}+\inf _{\boldsymbol{v}_{H} \in V_{H}^{s}}\left\|\partial_{t} \boldsymbol{\eta}\left(t^{i}\right)-\boldsymbol{v}_{H}\right\|_{V^{s}}\right. \\
\left.+\inf _{\boldsymbol{v}_{h} \in V_{h}^{f}}\left\|\boldsymbol{u}\left(t^{i}\right)-\boldsymbol{v}_{h}\right\|_{V^{f}}+C h^{l}\left\|\partial_{t} \boldsymbol{\eta}\left(t^{i}\right)\right\|_{V^{s}}\right)
\end{array}
$$

and

$$
\begin{gathered}
\left\|\boldsymbol{u}\left(t^{i}\right)-\boldsymbol{u}_{h}\left(t^{i}\right)\right\|_{1, \Omega^{f}} \leq C\left(\delta t\|\boldsymbol{\eta}\|_{W^{2, \infty}\left(0, T ; V^{s}\right)}+\inf _{\boldsymbol{v}_{H} \in V_{H}^{s}}\left\|\partial_{t} \boldsymbol{\eta}\left(t^{i}\right)-\boldsymbol{v}_{H}\right\|_{V^{s}}\right. \\
\left.+\inf _{\boldsymbol{v}_{h} \in V_{h}^{f}}\left\|\boldsymbol{u}\left(t^{i}\right)-\boldsymbol{v}_{h}\right\|_{1, \Omega^{f}}+\inf _{q_{h} \in Q_{h}}\left\|p\left(t^{i}\right)-q_{h}\right\|_{0, \Omega^{f}}+h^{l}\left\|\partial_{t} \boldsymbol{\eta}\left(t^{i}\right)\right\|_{V^{s}}\right)
\end{gathered}
$$

Finally, for the pressure we have exactly the same estimate, namely,

$$
\begin{gathered}
\left\|p\left(t^{i}\right)-p_{h}\left(t^{i}\right)\right\|_{1, \Omega^{f}} \leq C\left(\delta t\|\boldsymbol{\eta}\|_{W^{2, \infty}\left(0, T ; V^{s}\right)}+\inf _{\boldsymbol{v}_{H} \in V_{H}^{s}}\left\|\partial_{t} \boldsymbol{\eta}\left(t^{i}\right)-\boldsymbol{v}_{H}\right\|_{V^{s}}\right. \\
\left.+\inf _{\boldsymbol{v}_{h} \in V_{h}^{f}}\left\|\boldsymbol{u}\left(t^{i}\right)-\boldsymbol{v}_{h}\right\|_{1, \Omega^{f}}+\inf _{q_{h} \in Q_{h}}\left\|p\left(t^{i}\right)-q_{h}\right\|_{0, \Omega^{f}}+h^{l}\left\|\partial_{t} \boldsymbol{\eta}\left(t^{i}\right)\right\|_{H^{l}(\Sigma)}\right) .
\end{gathered}
$$

Again, $l$ verifies either (34) in the interpolation case or 35 in the mortar case.

\section{The error analysis}

Here we focus on the error analysis of the projection semi-implicit scheme for the velocity unknowns. The analysis will be performed assuming homogeneous Dirichlet conditions on the fluid velocity and on the structure displacement. As observed for example in 29, different boundary conditions could be used, provided we have at hand proper assumptions and regularity estimates. In the following, for the sake of conciseness, we will indicate with

$$
e_{h}^{k} \stackrel{\text { def }}{=} \boldsymbol{u}_{h}^{k}-\boldsymbol{u}_{h}\left(t^{k}\right), \quad \tilde{e}_{h}^{k} \stackrel{\text { def }}{=} \tilde{\boldsymbol{u}}_{h}^{k}-\boldsymbol{u}_{h}\left(t^{k}\right), \quad e_{H}^{k} \stackrel{\text { def }}{=} \boldsymbol{\eta}_{H}^{k}-\boldsymbol{\eta}_{H}\left(t^{k}\right),
$$

the errors associated with the fluid velocities and with the structure displacement. 


\subsection{Main result}

Theorem 2 Assume that hypotheses (20)-(21) (resp. (24)-(28)) are satisfied when $\pi_{h}$ is a finite element interpolation (resp. mortar) operator at the interface. Under the stability condition of the semi-implicit scheme, i.e.

$$
\rho^{s} \geq C\left(\rho^{f} \frac{h}{H^{\alpha}}+\frac{\mu \delta t}{h H^{\alpha}}\right), \quad \text { with } \quad \alpha= \begin{cases}0, & \text { if } \overline{\Omega^{s}}=\Sigma \\ 1, & \text { if } \overline{\Omega^{s}} \neq \Sigma\end{cases}
$$

and if the solution of the coupled problem (2)-(3) is smooth enough in space and time, then the discrete solution of (12)-(13) satisfies the following error estimate

$$
\begin{aligned}
\frac{\rho^{f}}{2}\left\|e_{h}^{n+1}\right\|_{0, \Omega^{f}}^{2}+\frac{\rho^{s}}{2}\left\|\frac{e_{H}^{n+1}-e_{H}^{n}}{\delta t}\right\|_{0, \Omega^{s}}^{2} & \frac{1}{4} a^{s}\left(e_{H}^{n+1}, e_{H}^{n+1}\right)+\sum_{n=0}^{N} \mu \delta t\left\|\boldsymbol{\epsilon}\left(\tilde{e}_{h}^{n+1}\right)\right\|_{0, \Omega^{f}}^{2} \\
& \leq C \delta t+C h^{2 k}+C H^{2 m}+C h^{2 l}, \quad(40)
\end{aligned}
$$

where $C$ denotes a strictly positive constant independent of $h, H$ and $\delta t$. Here $m$ depends on the choice of the finite element approximation space associated with the structure part and, for $\boldsymbol{v}^{s}$ smooth enough, is such that

$$
\inf _{\boldsymbol{v}_{H} \in V_{H}^{s}}\left\|\boldsymbol{v}^{s}-\boldsymbol{v}_{H}\right\|_{V^{s}} \leq C H^{m}
$$

while $l$ depends on the choice of the operator $\pi_{h}$, that is,

- $l \leq \min \left(r-\frac{\alpha}{2}, k+1\right)$ for interpolation type operator,

- $\frac{1}{2} \leq l \leq k$ for mortar type operator.

Before giving a proof of the previous theorem, a few remarks are in order. Considering the accuracy in time, the fluid-structure interaction scheme has globally a convergence order of $\sqrt{\delta t}$. As observed in the proof, this is due to the use of the non-incremental Chorin-Temam projection scheme in the fluid problem (which is known to satisfy (1) for the hydrodynamic problem) and to the semi-implicit coupling at the interface. Moreover, the optimality of the error estimate is strictly dependent on the interface operator used. As a matter of fact, the mortar matching will give, in any case, optimal error estimate, whereas the finite element interpolation operator will lead to an optimal error bound only in specific cases depending on the structure model and on the degree of the polynomial approximation for the fluid. For instance, if we consider a membrane, then $r=1$ and $\alpha=0$ and the error estimate will be optimal for $k=1$. Table 1 summarizes the different cases.

Proof. For the sake of clarity, due to its complexity, the proof will be split in several parts. Each one focuses on a particular point of the error estimate.

Part 1. The first step to obtain the error estimate (40) is to derive the equations verified by the errors $e_{h}^{k}, \tilde{e}_{h}^{k}$ and $e_{H}^{k}$.

Let us consider the fluid equation associated with the coupled problem (7). Choosing $\left(\boldsymbol{v}^{f}, q, \boldsymbol{v}^{s}\right)=\left(\boldsymbol{v}_{h}^{f}, q_{h}, 0\right)$, with $\left(\boldsymbol{v}^{f}, q\right) \in V_{h, 0}^{f} \times Q_{h}$, in (7) and recalling the definition of the finite element approximations $\left(\boldsymbol{u}_{h}\left(t^{n+1}\right), p_{h}\left(t^{n+1}\right)\right)$ of 


\begin{tabular}{|c|c|c|c|}
\hline$\pi_{h}$ & $\begin{array}{c}d \text {-dimensional } \\
\text { structure } \\
(r=1, \alpha=1)\end{array}$ & $\begin{array}{c}(d \text {-1)-dimensional } \\
\text { structure: } 2^{n d} \\
\text { order oper. } \\
(r=1, \alpha=0)\end{array}$ & $\begin{array}{c}(d \text {-1)-dimensional } \\
\text { structure: } 4^{t h} \\
\text { order oper. }( \\
r=2, \alpha=0)\end{array}$ \\
\hline Interpolation & non optimal & $k=1$ optimal & $k \leq 2$ optimal \\
\hline Mortar & optimal $\forall k$ & optimal $\forall k$ & optimal $\forall k$ \\
\hline
\end{tabular}

Table 1: Optimality of the error estimates depending on the structure model, the matching operator and the polynomial degree of the approximation of the fluid.

$$
\begin{aligned}
& \left(\boldsymbol{u}\left(t^{n+1}\right), p\left(t^{n+1}\right)\right), \text { we obtain } \\
& \rho^{f}\left(\frac{\boldsymbol{u}_{h}\left(t^{n+1}\right)-\boldsymbol{u}_{h}\left(t^{n}\right)}{\delta t}, \boldsymbol{v}_{h}^{f}\right)_{\Omega^{f}}+2 \mu\left(\boldsymbol{\epsilon}\left(\boldsymbol{u}_{h}\left(t^{n+1}\right)\right), \boldsymbol{\epsilon}\left(\boldsymbol{v}_{h}^{f}\right)\right)_{\Omega^{f}}-\left(p_{h}\left(t^{n+1}\right), \boldsymbol{\nabla} \cdot \boldsymbol{v}_{h}^{f}\right)_{\Omega^{f}} \\
& +\left(q_{h}, \boldsymbol{\nabla} \cdot \boldsymbol{u}_{h}\left(t^{n+1}\right)\right)_{\Omega^{f}}=\rho^{f}\left(\frac{\boldsymbol{u}_{h}\left(t^{n+1}\right)-\boldsymbol{u}_{h}\left(t^{n}\right)}{\delta t}-\partial_{t} \boldsymbol{u}\left(t^{n+1}\right), \boldsymbol{v}_{h}^{f}\right)_{\Omega^{f}} . \quad \text { (41) }
\end{aligned}
$$

Similarly, for the structure part, thanks to the definition of the finite element approximation $\boldsymbol{\eta}_{H}\left(t^{n+1}\right)$ of $\boldsymbol{\eta}\left(t^{n+1}\right)$ and choosing, in the variational formulation (7), $\left(\boldsymbol{v}^{f}, q, \boldsymbol{v}^{s}\right)=\left(\mathcal{L}_{h}\left(\boldsymbol{v}_{H}^{s}\right), 0, \boldsymbol{v}_{H}^{s}\right)$, such that $\boldsymbol{v}_{H}^{s} \in V_{H}^{s}$, we have

$$
\begin{aligned}
\rho^{s}\left(\frac{\boldsymbol{\eta}_{H}\left(t^{n+1}\right)-2 \boldsymbol{\eta}_{H}\left(t^{n}\right)+\boldsymbol{\eta}_{H}\left(t^{n-1}\right)}{\delta t^{2}}, \boldsymbol{v}_{H}^{s}\right)_{\Omega^{s}}+a^{s}\left(\boldsymbol{\eta}_{H}\left(t^{n+1}\right), \boldsymbol{v}_{H}^{s}\right) \\
=\rho^{s}\left(\frac{\boldsymbol{\eta}_{H}\left(t^{n+1}\right)-2 \boldsymbol{\eta}_{H}\left(t^{n}\right)+\boldsymbol{\eta}_{H}\left(t^{n-1}\right)}{\delta t^{2}}-\partial_{t t} \boldsymbol{\eta}\left(t^{n+1}\right), \boldsymbol{v}_{H}^{s}\right)_{\Omega^{s}} \\
-\left\langle\mathcal{R}\left(\boldsymbol{u}\left(t^{n+1}\right), p\left(t^{n+1}\right)\right), \mathcal{L}_{h}\left(\boldsymbol{v}_{H}^{s}\right)\right\rangle \\
+a^{s}\left(\boldsymbol{\eta}_{H}\left(t^{n+1}\right)-\boldsymbol{\eta}\left(t^{n+1}\right), \boldsymbol{v}_{H}^{s}\right)+\left\langle\xi\left(t^{n+1}\right), \boldsymbol{v}_{H}^{s}-\pi_{h}\left(\boldsymbol{v}_{H}^{s}\right)\right\rangle_{\Sigma} .
\end{aligned}
$$

The two equations are satisfied for all $t^{n+1} \leq T$. The last two terms on the right-hand side of (42) are respectively due to the introduction of the finite element approximation and to the non-conforming finite element discretization at the interface. The discrete lifting operator $\mathcal{L}_{h}: V_{H}^{s}(\Sigma) \rightarrow V_{h}^{f}$ verifies

$$
\mathcal{L}_{h}\left(\boldsymbol{v}_{H}^{s}\right)=\pi_{h}\left(\boldsymbol{v}_{H}^{s}\right), \quad \text { on } \quad \Sigma .
$$

The lifting $\mathcal{L}_{h}$ is chosen to be equal to $r_{h} \circ \pi_{h}$, where $r_{h}$ is a continuous linear lifting from $V_{h}^{f}(\Sigma)$ onto $V_{h}^{f}$ (for example defined thanks to a discrete Poisson problem), such that

$$
\left\|r_{h}\left(\boldsymbol{v}_{h}^{f}\right)\right\|_{1, \Omega^{f}} \leq C\left\|\boldsymbol{v}_{h}^{f}\right\|_{H_{00}^{\frac{1}{2}}(\Sigma)}, \forall \boldsymbol{v}_{h}^{f} \in V_{h}^{f}(\Sigma)
$$

Here the constant $C$ does not depend on the space discretization. Consequently, $\mathcal{L}_{h}$ satisfies

$$
\left\|\mathcal{L}_{h}\left(\boldsymbol{v}_{H}^{s}\right)\right\|_{1, \Omega^{f}} \leq C\left\|\pi_{h}\left(\boldsymbol{v}_{H}^{s}\right)\right\|_{H_{00}^{\frac{1}{2}}(\Sigma)}, \forall \boldsymbol{v}_{H}^{s} \in V_{H}^{s}(\Sigma)
$$


Subtracting equation 41 from $(16)_{1}$, we get the error equation associated with the first step of the semi-implicit scheme:

$$
\begin{aligned}
\forall & \left(\tilde{\boldsymbol{v}}_{h}^{f}, q_{h}\right) \in V_{h, 0}^{f} \times Q_{h}, \\
& \rho^{f}\left(\frac{\tilde{e}_{h}^{n+1}-e_{h}^{n}}{\delta t}, \tilde{\boldsymbol{v}}_{h}^{f}\right)_{\Omega^{f}}+2 \mu\left(\boldsymbol{\epsilon}\left(\tilde{e}_{h}^{n+1}\right), \boldsymbol{\epsilon}\left(\tilde{\boldsymbol{v}}_{h}^{f}\right)\right)_{\Omega^{f}}+\left(p_{h}\left(t^{n+1}\right), \boldsymbol{\nabla} \cdot \tilde{\boldsymbol{v}}_{h}^{f}\right)_{\Omega^{f}} \\
- & \left(q_{h}, \boldsymbol{\nabla} \cdot \boldsymbol{u}_{h}\left(t^{n+1}\right)\right)_{\Omega^{f}}=\rho^{f}\left(\partial_{t} \boldsymbol{u}\left(t^{n+1}\right)-\frac{\boldsymbol{u}_{h}\left(t^{n+1}\right)-\boldsymbol{u}_{h}\left(t^{n}\right)}{\delta t}, \tilde{\boldsymbol{v}}_{h}^{f}\right)_{\Omega^{f}} .
\end{aligned}
$$

The error equation associated with the fluid part of the second step can be obtained by rewriting $(17)_{1}$ in the following form:

$$
\begin{gathered}
\frac{\rho^{f}}{\delta t}\left(e_{h}^{n+1}-\tilde{e}_{h}^{n+1}, \boldsymbol{v}_{h}^{f}\right)_{\Omega^{f}}-\left(p_{h}^{n+1}, \boldsymbol{\nabla} \cdot \boldsymbol{v}_{h}^{f}\right)_{\Omega^{f}}+\left(q_{h}, \boldsymbol{\nabla} \cdot e_{h}^{n+1}\right)_{\Omega^{f}}+\left(q_{h}, \boldsymbol{\nabla} \cdot \boldsymbol{u}_{h}\left(t^{n+1}\right)\right)_{\Omega^{f}}=0, \\
\forall\left(\boldsymbol{v}_{h}^{f}, q_{h}\right) \in Y_{h, 0}^{f} \times Q_{h} .
\end{gathered}
$$

Finally, for the structure part, subtracting equation 42 from $(17)_{3}$ leads to

$$
\begin{aligned}
\rho^{s} & \left(\frac{e_{H}^{n+1}-2 e_{H}^{n}+e_{H}^{n-1}}{\delta t^{2}}, \boldsymbol{v}_{H}^{s}\right)_{\Omega^{s}}+a^{s}\left(e_{H}^{n+1}, \boldsymbol{v}_{H}^{s}\right) \\
& =\rho^{s}\left(\partial_{t t} \boldsymbol{\eta}\left(t^{n+1}\right)-\frac{\boldsymbol{\eta}_{H}\left(t^{n+1}\right)-2 \boldsymbol{\eta}_{H}\left(t^{n}\right)+\boldsymbol{\eta}_{H}\left(t^{n-1}\right)}{\delta t^{2}}, \boldsymbol{v}_{H}^{s}\right)_{\Omega^{s}}+\mathcal{T}_{h}^{n+1} \\
& -a^{s}\left(\boldsymbol{\eta}_{H}\left(t^{n+1}\right)-\boldsymbol{\eta}\left(t^{n+1}\right), \boldsymbol{v}_{H}^{s}\right)-\left\langle\xi\left(t^{n+1}\right), \boldsymbol{v}_{H}^{s}-\pi_{h}\left(\boldsymbol{v}_{H}^{s}\right)\right\rangle_{\Sigma}, \quad \forall \boldsymbol{v}_{H}^{s} \in V_{H}^{s},
\end{aligned}
$$

where

$$
\begin{aligned}
\mathcal{T}_{h}^{n+1} \stackrel{\text { def }}{=} & \left\langle\mathcal{R}\left(\boldsymbol{u}\left(t^{n+1}\right), p\left(t^{n+1}\right)\right), \mathcal{L}_{h}\left(\boldsymbol{v}_{H}^{s}\right)\right\rangle-\left\langle\mathcal{R}_{\mu}\left(\tilde{\boldsymbol{u}}_{h}^{n+1}\right), \mathcal{L}_{h}\left(\boldsymbol{v}_{H}^{s}\right)\right\rangle \\
& -\left\langle\mathcal{R}_{p}\left(\boldsymbol{u}_{h}^{n+1}, p_{h}^{n+1}\right), \mathcal{L}_{h}\left(\boldsymbol{v}_{H}^{s}\right)\right\rangle \\
= & \rho^{f}\left(\partial_{t} \boldsymbol{u}\left(t^{n+1}\right), \mathcal{L}_{h}\left(\boldsymbol{v}_{H}^{s}\right)\right)_{\Omega^{f}}+2 \mu\left(\boldsymbol{\epsilon}\left(\boldsymbol{u}\left(t^{n+1}\right)\right), \boldsymbol{\epsilon}\left(\mathcal{L}_{h}\left(\boldsymbol{v}_{H}^{s}\right)\right)\right)_{\Omega^{f}} \\
& -\left(p\left(t^{n+1}\right), \boldsymbol{\nabla} \cdot \mathcal{L}_{h}\left(\boldsymbol{v}_{H}^{s}\right)\right)_{\Omega^{f}}-\rho^{f}\left(\frac{\tilde{\boldsymbol{u}}_{h}^{n+1}-\boldsymbol{u}_{h}^{n}}{\delta t}, \mathcal{L}_{h}\left(\boldsymbol{v}_{H}^{s}\right)\right)_{\Omega^{f}} \\
& -2 \mu\left(\boldsymbol{\epsilon}\left(\tilde{\boldsymbol{u}}_{h}^{n+1}\right), \boldsymbol{\epsilon}\left(\mathcal{L}_{h}\left(\boldsymbol{v}_{H}^{s}\right)\right)\right)_{\Omega^{f}}-\rho^{f}\left(\frac{\boldsymbol{u}_{h}^{n+1}-\tilde{\boldsymbol{u}}_{h}^{n+1}}{\delta t}, \mathcal{L}_{h}\left(\boldsymbol{v}_{H}^{s}\right)\right)_{\Omega^{f}} \\
& +\left(p_{h}^{n+1}, \boldsymbol{\nabla} \cdot \mathcal{L}_{h}\left(\boldsymbol{v}_{H}^{s}\right)\right)_{\Omega^{f}} \\
= & \rho^{f}\left(\partial_{t} \boldsymbol{u}\left(t^{n+1}\right)-\frac{\boldsymbol{u}_{h}\left(t^{n+1}\right)-\boldsymbol{u}_{h}\left(t^{n}\right)}{\delta t}, \mathcal{L}_{h}\left(\boldsymbol{v}_{H}^{s}\right)\right)_{\Omega^{f}} \\
& -\rho^{f}\left(\frac{e_{h}^{n+1}-e_{h}^{n}}{\delta t}, \mathcal{L}_{h}\left(\boldsymbol{v}_{H}^{s}\right)\right)_{\Omega^{f}}-2 \mu\left(\boldsymbol{\epsilon}\left(\tilde{e}_{h}^{n+1}\right), \boldsymbol{\epsilon}\left(\mathcal{L}_{h}\left(\boldsymbol{v}_{H}^{s}\right)\right)\right)_{\Omega^{f}} \\
& +2 \mu\left(\boldsymbol{\epsilon}\left(\boldsymbol{u}\left(t^{n+1}\right)-\boldsymbol{u}_{h}\left(t^{n+1}\right)\right), \boldsymbol{\epsilon}\left(\mathcal{L}_{h}\left(\boldsymbol{v}_{H}^{s}\right)\right)\right)_{\Omega^{f}}+\left(p_{h}^{n+1}-p\left(t^{n+1}\right), \boldsymbol{\nabla} \cdot \mathcal{L}_{h}\left(\boldsymbol{v}_{H}^{s}\right)\right)_{\Omega^{f}} .
\end{aligned}
$$


Part 2. We now add (45), (46) and (47) and test them using the following test functions:

$$
\begin{array}{r}
\boldsymbol{v}_{H}^{s}=e_{H}^{n+1}-e_{H}^{n}, \quad \boldsymbol{v}_{h}^{f}=\delta t\left(e_{h}^{n+1}-\mathcal{L}_{h}\left(\frac{\boldsymbol{v}_{H}^{s}}{\delta t}\right)\right), \quad q_{h}=0 \\
\tilde{\boldsymbol{v}}_{h}^{f}=\delta t\left(\tilde{e}_{h}^{n+1}-\mathcal{L}_{h}\left(\frac{\boldsymbol{v}_{H}^{s}}{\delta t}\right)+\mathcal{L}_{h}^{\prime}\left(\frac{e_{H}^{n+1}-2 e_{H}^{n}+e_{H}^{n-1}}{\delta t}\right)\right. \\
\left.+\mathcal{L}_{h}^{\prime}\left(\frac{\boldsymbol{\eta}_{H}\left(t^{n+1}\right)-2 \boldsymbol{\eta}_{H}\left(t^{n}\right)+\boldsymbol{\eta}_{H}\left(t^{n-1}\right)}{\delta t}\right)\right),
\end{array}
$$

where $\mathcal{L}_{h}^{\prime}$ is a second discrete lifting operator, satisfying $\mathcal{L}_{h}^{\prime}\left(\boldsymbol{v}_{H}\right)=\pi_{h}\left(\boldsymbol{v}_{H}^{s}\right)$ on $\Sigma$. It is defined as in [20] by:

$$
\mathcal{L}_{h}^{\prime}\left(\boldsymbol{b}_{H}\right) \stackrel{\text { def }}{=} \sum_{i=1, x_{h}^{i} \in \Sigma}^{N_{h}} \pi_{h}\left(\boldsymbol{b}_{H}\right)\left(x_{h}^{i}\right) \phi_{h}^{i},
$$

with $\left\{x_{h}^{i}\right\}_{i=1}^{N_{h}},\left\{\phi_{h}^{i}\right\}_{i=1}^{N_{h}}$ the sets of nodes and shape functions of $V_{h}^{f}$. We distinguish the latter from $\mathcal{L}_{h}$ since the two have different properties. This new lifting satisfies the following lemma (proved in [20])

Lemma 1 If the interface operator $\pi_{h}$ is stable in the $L^{2}$-norm on $V_{H}^{s}(\Sigma)$, then there exists a constant $C>0$, depending only on the local mesh geometry and on the polynomial order, such that:

$$
\begin{gathered}
\left\|\mathcal{L}_{h}^{\prime}\left(\boldsymbol{b}_{H}\right)\right\|_{0, \Omega^{f}}^{2} \leq \frac{C h}{H^{\alpha}}\left\|\boldsymbol{b}_{H}\right\|_{0, \Omega^{s}}^{2}, \\
\left\|\boldsymbol{\nabla} \mathcal{L}_{h}^{\prime}\left(\boldsymbol{b}_{H}\right)\right\|_{0, \Omega^{f}}^{2} \leq \frac{C}{h H^{\alpha}}\left\|\boldsymbol{b}_{H}\right\|_{0, \Omega^{s}}^{2},
\end{gathered}
$$

for all $\boldsymbol{b}_{H} \in V_{H}^{s}$, and $\alpha$ given by 22 .

Remark 4 The last two terms in the definition of the test function $\tilde{\boldsymbol{v}}_{h}^{f}$ represent an appropriate correction, added because $\tilde{\boldsymbol{u}}_{h}^{n+1}$ is equal to the structure velocity at the previous time step at the interface (explicit coupling). This guarantees the admissibility of the test functions.

After some direct simplifications, we have

$$
\begin{array}{r}
\rho^{f}\left(\tilde{e}_{h}^{n+1}-e_{h}^{n}, \tilde{e}_{h}^{n+1}\right)_{\Omega^{f}}+2 \mu \delta t\left\|\boldsymbol{\epsilon}\left(\tilde{e}_{h}^{n+1}\right)\right\|_{0, \Omega^{f}}^{2}+\rho^{f}\left(e_{h}^{n+1}-\tilde{e}_{h}^{n+1}, e_{h}^{n+1}\right)_{\Omega^{f}} \\
+\rho^{s}\left(\frac{e_{H}^{n+1}-2 e_{H}^{n}+e_{H}^{n-1}}{\delta t^{2}}, e_{H}^{n+1}-e_{H}^{n}\right)_{\Omega^{s}}+a^{s}\left(e_{H}^{n+1}, e_{H}^{n+1}-e_{H}^{n}\right) \\
+\mathcal{P}_{h}^{n+1}+\mathcal{C}_{h}^{n+1}+\mathcal{S}_{h}^{n+1}=\mathcal{D}_{h}^{n+1}+\mathcal{A}_{h}^{n+1} .
\end{array}
$$

In the left-hand side $\mathcal{P}_{h}^{n+1}, \mathcal{C}_{h}^{n+1}$ and $\mathcal{S}_{h}^{n+1}$ are respectively defined by

$$
\begin{aligned}
\mathcal{P}_{h}^{n+1} \stackrel{\text { def }}{=} & \delta t\left(p_{h}\left(t^{n+1}\right), \boldsymbol{\nabla} \cdot\left(\tilde{e}_{h}^{n+1}-e_{h}^{n+1}\right)\right)_{\Omega^{f}} \\
& +\delta t\left(p_{h}\left(t^{n+1}\right), \boldsymbol{\nabla} \cdot \mathcal{L}_{h}^{\prime}\left(\frac{e_{H}^{n+1}-2 e_{H}^{n}+e_{H}^{n-1}}{\delta t}\right)\right)_{\Omega^{f}} \\
& +\delta t\left(p_{h}\left(t^{n+1}\right), \boldsymbol{\nabla} \cdot \mathcal{L}_{h}^{\prime}\left(\frac{\boldsymbol{\eta}_{H}\left(t^{n+1}\right)-2 \boldsymbol{\eta}_{H}\left(t^{n}\right)+\boldsymbol{\eta}_{H}\left(t^{n-1}\right)}{\delta t}\right)\right)_{\Omega^{f}}(50)
\end{aligned}
$$




$$
\begin{aligned}
\mathcal{C}_{h}^{n+1} \stackrel{\text { def }}{=} \rho^{f}\left(\tilde{e}_{h}^{n+1}-e_{h}^{n}, \mathcal{L}_{h}^{\prime}\left(\frac{\boldsymbol{\eta}_{H}\left(t^{n+1}\right)-2 \boldsymbol{\eta}_{H}\left(t^{n}\right)+\boldsymbol{\eta}_{H}\left(t^{n-1}\right)}{\delta t}\right)\right)_{\Omega^{f}} \\
+2 \mu \delta t\left(\boldsymbol{\epsilon}\left(\tilde{e}_{h}^{n+1}\right), \boldsymbol{\epsilon}\left(\mathcal{L}_{h}^{\prime}\left(\frac{\boldsymbol{\eta}_{H}\left(t^{n+1}\right)-2 \boldsymbol{\eta}_{H}\left(t^{n}\right)+\boldsymbol{\eta}_{H}\left(t^{n-1}\right)}{\delta t}\right)\right)\right)(51,) \\
\mathcal{S}_{h}^{n+1} \stackrel{\text { def }}{=} \rho_{\Omega^{f}}^{f}\left(\tilde{e}_{h}^{n+1}-e_{h}^{n}, \mathcal{L}_{h}^{\prime}\left(\frac{e_{H}^{n+1}-2 e_{H}^{n}+e_{H}^{n-1}}{\delta t}\right)\right)_{\Omega^{f}} \\
+2 \mu \delta t\left(\boldsymbol{\epsilon}\left(\tilde{e}_{h}^{n+1}\right), \boldsymbol{\epsilon}\left(\mathcal{L}_{h}^{\prime}\left(\frac{e_{H}^{n+1}-2 e_{H}^{n}+e_{H}^{n-1}}{\delta t}\right)\right)\right)_{\Omega^{f}} .
\end{aligned}
$$

The term $\mathcal{P}_{h}^{n+1}$ regroups all the parts related to the pressure $p_{h}\left(t^{n+1}\right)$. In $\mathcal{C}_{h}^{n+1}$ we put the terms associated with the semi-implicit coupling (see the following remark for more details), while in $\mathcal{S}_{h}^{n+1}$ there are the terms that will be controlled by means of the stability condition 19 .

Remark 5 The discrete fluid velocities, $\tilde{\boldsymbol{u}}_{h}^{n+1}$ and $\boldsymbol{u}_{h}^{n+1}$, verify respectively an explicit and an implicit interface condition, while the finite element approximation of the continuous solution $\boldsymbol{u}$ is based only on the implicit one. This difference generate therefore terms like the ones in (51). By the way, we also observe that the last term in (50) is of the same kind. Note that similar terms would appear using a finite element approximation of $\boldsymbol{u}$ satisfying an explicit coupling condition.

Finally, considering the right-hand side, in $\mathcal{D}_{h}^{n+1}$ we include terms in which a time consistency error has to be estimated, that is

$$
\begin{aligned}
\mathcal{D}_{h}^{n+1} \stackrel{\text { def }}{=} & \rho^{f} \delta t\left(\partial_{t} \boldsymbol{u}\left(t^{n+1}\right)-\frac{\boldsymbol{u}_{h}\left(t^{n+1}\right)-\boldsymbol{u}_{h}\left(t^{n}\right)}{\delta t}, \tilde{e}_{h}^{n+1}\right)_{\Omega^{f}} \\
& +\rho^{f} \delta t\left(\partial_{t} \boldsymbol{u}\left(t^{n+1}\right)-\frac{\boldsymbol{u}_{h}\left(t^{n+1}\right)-\boldsymbol{u}_{h}\left(t^{n}\right)}{\delta t}, \mathcal{L}_{h}^{\prime}\left(\frac{e_{H}^{n+1}-2 e_{H}^{n}+e_{H}^{n-1}}{\delta t}\right)\right)_{\Omega^{f}} \\
& +\rho^{f} \delta t\left(\partial_{t} \boldsymbol{u}\left(t^{n+1}\right)-\frac{\boldsymbol{u}_{h}\left(t^{n+1}\right)-\boldsymbol{u}_{h}\left(t^{n}\right)}{\delta t}, \mathcal{L}_{h}^{\prime}\left(\frac{\boldsymbol{\eta}_{H}\left(t^{n+1}\right)-2 \boldsymbol{\eta}_{H}\left(t^{n}\right)+\boldsymbol{\eta}_{H}\left(t^{n-1}\right)}{\delta t}\right)\right)_{\Omega^{f}} \\
& +\rho^{s}\left(\partial_{t t} \boldsymbol{\eta}\left(t^{n+1}\right)-\frac{\boldsymbol{\eta}_{H}\left(t^{n+1}\right)-2 \boldsymbol{\eta}_{H}\left(t^{n}\right)+\boldsymbol{\eta}_{H}\left(t^{n-1}\right)}{\delta t^{2}}, e_{H}^{n+1}-e_{H}^{n}\right)_{\Omega^{s}} ;
\end{aligned}
$$

while $\mathcal{A}_{h}^{n+1}$ regroups terms in which a space consistency error has to be estimated (depending on the finite element approximation of the continuous solution we built):

$$
\begin{aligned}
& \mathcal{A}_{h}^{n+1} \stackrel{\text { def }}{=}-a^{s}\left(\boldsymbol{\eta}_{H}\left(t^{n+1}\right)-\boldsymbol{\eta}\left(t^{n+1}\right), e_{H}^{n+1}-e_{H}^{n}\right)-\left\langle\xi\left(t^{n+1}\right), e_{H}^{n+1}-e_{H}^{n}-\pi_{h}\left(e_{H}^{n+1}-e_{H}^{n}\right)\right\rangle_{\Sigma} \\
& +2 \mu\left(\boldsymbol{\epsilon}\left(\boldsymbol{u}\left(t^{n+1}\right)-\boldsymbol{u}_{h}\left(t^{n+1}\right)\right), \boldsymbol{\epsilon}\left(\mathcal{L}_{h}\left(e_{H}^{n+1}-e_{H}^{n}\right)\right)\right)_{\Omega^{f}} \\
& +\delta t\left(p_{h}\left(t^{n+1}\right)-p\left(t^{n+1}\right), \nabla \cdot \mathcal{L}_{h}\left(\frac{e_{H}^{n+1}-e_{H}^{n}}{\delta t}\right)\right)_{\Omega^{f}} .
\end{aligned}
$$

In 49 , most of the terms involving the continuous lifting $\mathcal{L}_{h}$ cancel each other except for the two appearing in $\mathcal{A}_{h}^{n+1}$. The cancellation is due to the fact that they are linked to the energy balance of the system at the interface. 
Next we set

$$
\begin{aligned}
\mathcal{E}_{h}^{n+1} \stackrel{\text { def }}{=} & \rho^{f}\left(\tilde{e}_{h}^{n+1}-e_{h}^{n}, \tilde{e}_{h}^{n+1}\right)_{\Omega^{f}}+2 \mu \delta t\left\|\boldsymbol{\epsilon}\left(\tilde{e}_{h}^{n+1}\right)\right\|_{0, \Omega^{f}}^{2}+\rho^{f}\left(e_{h}^{n+1}-\tilde{e}_{h}^{n+1}, e_{h}^{n+1}\right)_{\Omega^{f}} \\
& +\rho^{s}\left(\frac{e_{H}^{n+1}-2 e_{H}^{n}+e_{H}^{n-1}}{\delta t^{2}}, e_{H}^{n+1}-e_{H}^{n}\right)_{\Omega^{s}}+a^{s}\left(e_{H}^{n+1}, e_{H}^{n+1}-e_{H}^{n}\right) .
\end{aligned}
$$

In $\mathcal{E}_{h}^{n+1}$ we collect all the terms associated with the energy of the system. Using the identity $(a-b, a)=\frac{1}{2}\|a\|^{2}-\frac{1}{2}\|b\|^{2}+\frac{1}{2}\|a-b\|^{2}$, the bi-linearity and the symmetry of $a^{s}(\cdot, \cdot)$ and introducing the quantity

$$
\tilde{E}^{n+1} \stackrel{\text { def }}{=} \frac{\rho^{f}}{2}\left\|e_{h}^{n+1}\right\|_{0, \Omega^{f}}^{2}+\frac{\rho^{s}}{2}\left\|\frac{e_{H}^{n+1}-e_{H}^{n}}{\delta t}\right\|_{0, \Omega^{s}}^{2}+\frac{1}{2} a^{s}\left(e_{H}^{n+1}, e_{H}^{n+1}\right),
$$

we have

$$
\begin{aligned}
\mathcal{E}_{h}^{n+1}= & \frac{\rho^{f}}{2}\left[\left\|e_{h}^{n+1}\right\|_{0, \Omega^{f}}^{2}-\left\|e_{h}^{n}\right\|_{0, \Omega^{f}}^{2}+\left\|\tilde{e}_{h}^{n+1}-e_{h}^{n}\right\|_{0, \Omega^{f}}^{2}+\left\|e_{h}^{n+1}-\tilde{e}_{h}^{n+1}\right\|_{0, \Omega^{f}}^{2}\right] \\
& +\frac{\rho^{s}}{2}\left[\left\|\frac{e_{H}^{n+1}-e_{H}^{n}}{\delta t}\right\|_{0, \Omega^{s}}^{2}-\left\|\frac{e_{H}^{n}-e_{H}^{n-1}}{\delta t}\right\|_{0, \Omega^{s}}^{2}+\| \frac{e_{H}^{n+1}-2 e_{H}^{n}+e_{H}^{n-1} \|_{0, \Omega^{s}}^{2}}{\delta t}\right] \\
& +\frac{1}{2}\left[a^{s}\left(e_{H}^{n+1}, e_{H}^{n+1}\right)-a^{s}\left(e_{H}^{n}, e_{H}^{n}\right)+a^{s}\left(e_{H}^{n+1}-e_{H}^{n}, e_{H}^{n+1}-e_{H}^{n}\right)\right]+2 \mu \delta t\left\|\boldsymbol{\epsilon}\left(\tilde{e}_{h}^{n+1}\right)\right\|_{0, \Omega^{f}}^{2} \\
= & \tilde{E}^{n+1}-\tilde{E}^{n}+\frac{\rho^{f}}{2}\left[\left\|\tilde{e}_{h}^{n+1}-e_{h}^{n}\right\|_{0, \Omega^{f}}^{2}+\left\|e_{h}^{n+1}-e_{h}^{n}\right\|_{0, \Omega^{f}}^{2}\right]+2 \mu \delta t\left\|\boldsymbol{\epsilon}\left(\tilde{e}_{h}^{n+1}\right)\right\|_{0, \Omega^{f}}^{2} \\
& +\frac{\rho^{s}}{2}\left\|\frac{e_{H}^{n+1}-2 e_{H}^{n}+e_{H}^{n-1}}{\delta t}\right\|_{0, \Omega^{s}}^{2}+\frac{1}{2} a^{s}\left(e_{H}^{n+1}-e_{H}^{n}, e_{H}^{n+1}-e_{H}^{n}\right) .
\end{aligned}
$$

Making use of the equality (56) in (49) and reordering the different terms, we get

$$
\begin{aligned}
\tilde{E}^{n+1} & +\frac{\rho^{f}}{2}\left[\left\|\tilde{e}_{h}^{n+1}-e_{h}^{n}\right\|_{0, \Omega^{f}}^{2}+\left\|e_{h}^{n+1}-\tilde{e}_{h}^{n+1}\right\|_{0, \Omega^{f}}^{2}\right]+2 \mu \delta t\left\|\boldsymbol{\epsilon}\left(\tilde{e}_{h}^{n+1}\right)\right\|_{0, \Omega^{f}}^{2} \\
& +\frac{\rho^{s}}{2}\left\|\frac{e_{H}^{n+1}-2 e_{H}^{n}+e_{H}^{n-1}}{\delta t}\right\|_{0, \Omega^{s}}^{2}+\frac{1}{2} a^{s}\left(e_{H}^{n+1}-e_{H}^{n}, e_{H}^{n+1}-e_{H}^{n}\right) \\
& =\tilde{E}^{n}-\mathcal{P}_{h}^{n+1}-\mathcal{C}_{h}^{n+1}-\mathcal{S}_{h}^{n+1}+\mathcal{D}_{h}^{n+1}+\mathcal{A}_{h}^{n+1}
\end{aligned}
$$

Part 3. We now give an upper bound for each term in the right-hand side of 57. In the following we will frequently make use of the classical Young's inequality

$$
a b \leq \frac{\gamma}{2} a^{2}+\frac{1}{2 \gamma} b^{2} \quad \forall \gamma>0,
$$

that will be simply replaced by

$$
a b \leq \frac{\gamma}{2} a^{2}+C b^{2},
$$

or by

$$
a b \leq C\left(a^{2}+b^{2}\right),
$$


whenever the definition of the constant $\gamma$ for one (or both) of the terms doesn't play a key role in the convergence analysis. The quantity $C$ represents a positive constant.

Let us consider $\mathcal{P}_{h}^{n+1}$. First we integrate by parts all its terms:

$$
\begin{aligned}
-\mathcal{P}_{h}^{n+1}= & \delta t\left(\nabla p_{h}\left(t^{n+1}\right), \tilde{e}_{h}^{n+1}-e_{h}^{n+1}\right)_{\Omega^{f}} \\
& +\delta t\left(\nabla p_{h}\left(t^{n+1}\right), \mathcal{L}_{h}^{\prime}\left(\frac{e_{H}^{n+1}-2 e_{H}^{n}+e_{H}^{n-1}}{\delta t}\right)\right)_{\Omega^{f}} \\
& +\delta t\left(\nabla p_{h}\left(t^{n+1}\right), \mathcal{L}_{h}^{\prime}\left(\frac{\boldsymbol{\eta}_{H}\left(t^{n+1}\right)-2 \boldsymbol{\eta}_{H}\left(t^{n}\right)+\boldsymbol{\eta}_{H}\left(t^{n-1}\right)}{\delta t}\right)\right)_{\Omega^{f}}(60)
\end{aligned}
$$

note that in the latter no extra boundary terms were introduced since

$$
\left.\left(e_{h}^{n+1}-\tilde{e}_{h}^{n+1}\right) \cdot \mathbf{n}\right|_{\Sigma}=\left.\pi_{h}\left(\frac{\boldsymbol{\eta}_{H}^{n+1}-2 \boldsymbol{\eta}_{H}^{n}+\boldsymbol{\eta}_{H}^{n-1}}{\delta t}\right) \cdot \mathbf{n}\right|_{\Sigma} .
$$

Then, the Young's inequality and the properties 48 of $\mathcal{L}_{h}^{\prime}$ yield

$$
\begin{aligned}
& -\mathcal{P}_{h}^{n+1} \leq \frac{\gamma_{1} \rho^{f}}{2}\left\|e_{h}^{n+1}-\tilde{e}_{h}^{n+1}\right\|_{0, \Omega^{f}}^{2}+\frac{C h \rho^{f}}{H^{\alpha}}\left\|\frac{e_{H}^{n+1}-2 e_{H}^{n}+e_{H}^{n-1}}{\delta t}\right\|_{0, \Omega^{s}}^{2} \\
& +\frac{C h \rho^{f}}{H^{\alpha}}\left\|\frac{\boldsymbol{\eta}_{H}\left(t^{n+1}\right)-2 \boldsymbol{\eta}_{H}\left(t^{n}\right)+\boldsymbol{\eta}_{H}\left(t^{n-1}\right)}{\delta t}\right\|_{0, \Omega^{s}}^{2}+\frac{C \delta t^{2}}{\rho^{f}}\left\|\nabla p_{h}\left(t^{n+1}\right)\right\|_{0, \Omega^{f}}^{2} .
\end{aligned}
$$

In a similar way, for $\mathcal{C}_{h}^{n+1}$ and $\mathcal{S}_{h}^{n+1}$, defined respectively by 51 and 52 , using (58) and the properties 48 of $\mathcal{L}_{h}^{\prime}$, we get

$$
\begin{aligned}
-\mathcal{C}_{h}^{n+1} \leq & \frac{\gamma_{2} \rho^{f}}{2}\left\|\tilde{e}_{h}^{n+1}-e_{h}^{n}\right\|_{0, \Omega^{f}}^{2}+\gamma_{3} \mu \delta t\left\|\boldsymbol{\epsilon}\left(\tilde{e}_{h}^{n+1}\right)\right\|_{0, \Omega^{f}}^{2} \\
& +C\left(\frac{h \rho^{f}}{H^{\alpha}}+\frac{\mu \delta t}{h H^{\alpha}}\right)\left\|\frac{\boldsymbol{\eta}_{H}\left(t^{n+1}\right)-2 \boldsymbol{\eta}_{H}\left(t^{n}\right)+\boldsymbol{\eta}_{H}\left(t^{n-1}\right)}{\delta t}\right\|_{0, \Omega^{s}}^{2}, \\
-\mathcal{S}_{h}^{n+1} \leq \frac{\gamma_{1} \rho^{f}}{2}\left\|e_{h}^{n+1}-\tilde{e}_{h}^{n+1}\right\|_{0, \Omega^{f}}^{2}+\gamma_{3} \mu \delta t\left\|\boldsymbol{\epsilon}\left(\tilde{e}_{h}^{n+1}\right)\right\|_{0, \Omega^{f}}^{2} & \quad C\left(\frac{h \rho^{f}}{H^{\alpha}}+\frac{\mu \delta t}{h H^{\alpha}}\right)\left\|\frac{e_{H}^{n+1}-2 e_{H}^{n}+e_{H}^{n-1}}{\delta t}\right\|_{0, \Omega^{s}}^{2} .
\end{aligned}
$$

For $\mathcal{D}_{h}^{n+1}$, defined by 53 , we rewrite the first term as

$$
\begin{aligned}
\rho^{f} \delta t\left(\partial_{t} \boldsymbol{u}\left(t^{n+1}\right)-\right. & \left.\frac{\boldsymbol{u}_{h}\left(t^{n+1}\right)-\boldsymbol{u}_{h}\left(t^{n}\right)}{\delta t}, \tilde{e}_{h}^{n+1}\right)_{\Omega^{f}}= \\
\rho^{f} \delta t\left(\partial_{t} \boldsymbol{u}\left(t^{n+1}\right)-\frac{\boldsymbol{u}_{h}\left(t^{n+1}\right)-\boldsymbol{u}_{h}\left(t^{n}\right)}{\delta t}, \tilde{e}_{h}^{n+1}-e_{h}^{n+1}\right)_{\Omega^{f}} & \\
& +\rho^{f} \delta t\left(\partial_{t} \boldsymbol{u}\left(t^{n+1}\right)-\frac{\boldsymbol{u}_{h}\left(t^{n+1}\right)-\boldsymbol{u}_{h}\left(t^{n}\right)}{\delta t}, e_{h}^{n+1}\right)_{\Omega^{f}},
\end{aligned}
$$


then, the use of (59) and once again of the properties 48 yield after some rearrangements

$$
\begin{gathered}
\mathcal{D}_{h}^{n+1} \leq C \rho^{f} \delta t\left(\left\|\tilde{e}_{h}^{n+1}-e_{h}^{n+1}\right\|_{0, \Omega^{f}}^{2}+\left\|e_{h}^{n+1}\right\|_{0, \Omega^{f}}^{2}\right)+\frac{C h \rho^{f} \delta t}{H^{\alpha}}\left\|\frac{e_{H}^{n+1}-2 e_{H}^{n}+e_{H}^{n-1}}{\delta t}\right\|_{0, \Omega^{s}}^{2} \\
+\frac{C h \rho^{f} \delta t}{H^{\alpha}}\left\|\frac{\boldsymbol{\eta}_{H}\left(t^{n+1}\right)-2 \boldsymbol{\eta}_{H}\left(t^{n}\right)+\boldsymbol{\eta}_{H}\left(t^{n-1}\right)}{\delta t}\right\|_{0, \Omega^{s}}^{2}+C \rho^{s} \delta t\left\|\frac{e_{H}^{n+1}-e_{H}^{n}}{\delta t}\right\|_{0, \Omega^{s}}^{2} \\
+C \rho^{f} \delta t\left\|\partial_{t} \boldsymbol{u}\left(t^{n+1}\right)-\frac{\boldsymbol{u}_{h}\left(t^{n+1}\right)-\boldsymbol{u}_{h}\left(t^{n}\right)}{\delta t}\right\|_{0, \Omega^{f}}^{2} \\
+C \rho^{s} \delta t\left\|\partial_{t t} \boldsymbol{\eta}\left(t^{n+1}\right)-\frac{\boldsymbol{\eta}_{H}\left(t^{n+1}\right)-2 \boldsymbol{\eta}_{H}\left(t^{n}\right)+\boldsymbol{\eta}_{H}\left(t^{n-1}\right)}{\delta t^{2}}\right\|_{0, \Omega^{s}}^{2} .
\end{gathered}
$$

Remark 6 Instead of rewriting the first term as in (64), another possibility is to apply first the Young's inequality (59) and then control $\left\|\tilde{e}_{h}^{n+1}\right\|_{0, \Omega^{f}}^{2}$ thanks to the Korn's inequality.

Before estimating the four terms of $\mathcal{A}_{h}^{n+1}$, we first apply to the error equation (57) the estimates 61, 62, 63) and 65), respectively obtained for $\mathcal{P}_{h}^{n+1}$, $\mathcal{C}_{h}^{n+1}, \mathcal{S}_{h}^{n+1}$ and $\mathcal{D}_{h}^{n+1}$. Then, after adding up from $n=0$ to $N$, where $N$ is a positive integer such that $(N+1) \delta t \leq T$, and rearranging the different terms, we obtain

$$
\begin{aligned}
& \tilde{E}^{N+1}+\frac{\rho^{f}}{2} \sum_{n=0}^{N}\left[\left(1-\gamma_{2}\right)\left\|\tilde{e}_{h}^{n+1}-e_{h}^{n}\right\|_{0, \Omega^{f}}^{2}+\left(1-2 \gamma_{1}\right)\left\|e_{h}^{n+1}-\tilde{e}_{h}^{n+1}\right\|_{0, \Omega^{f}}^{2}\right] \\
& +\sum_{n=0}^{N} 2 \mu \delta t\left(1-\gamma_{3}\right)\left\|\boldsymbol{\epsilon}\left(\tilde{e}_{h}^{n+1}\right)\right\|_{0, \Omega^{f}}^{2}+\frac{1}{2} \sum_{n=0}^{N} a^{s}\left(e_{H}^{n+1}-e_{H}^{n}, e_{H}^{n+1}-e_{H}^{n}\right) \\
& +\sum_{n=0}^{N}\left(\frac{\rho^{s}}{2}-\frac{C h \rho^{f}}{H^{\alpha}}-\frac{C \mu \delta t}{h H^{\alpha}}\right)\left\|\frac{e_{H}^{n+1}-2 e_{H}^{n}+e_{H}^{n-1}}{\delta t}\right\|_{0, \Omega^{s}}^{2} \\
& \leq \tilde{E}^{0}+\sum_{n=0}^{N} C\left(\frac{h \rho^{f}}{H^{\alpha}}+\frac{\mu \delta t}{h H^{\alpha}}\right)\left\|\frac{\boldsymbol{\eta}_{H}\left(t^{n+1}\right)-2 \boldsymbol{\eta}_{H}\left(t^{n}\right)+\boldsymbol{\eta}_{H}\left(t^{n-1}\right)}{\delta t}\right\|_{0, \Omega^{s}}^{2} \\
& +\sum_{n=0}^{N} \frac{C \delta t^{2}}{\rho^{f}}\left\|\boldsymbol{\nabla} p_{h}\left(t^{n+1}\right)\right\|_{0, \Omega^{f}}^{2}+\sum_{n=0}^{N} C \frac{h \rho^{f} \delta t}{H^{\alpha}}\left\|\frac{\boldsymbol{\eta}_{H}\left(t^{n+1}\right)-2 \boldsymbol{\eta}_{H}\left(t^{n}\right)+\boldsymbol{\eta}_{H}\left(t^{n-1}\right)}{\delta t}\right\|_{0, \Omega^{s}}^{2} \\
& +\sum_{n=0}^{N} C \rho^{f} \delta t\left(\left\|\tilde{e}_{h}^{n+1}-e_{h}^{n+1}\right\|_{0, \Omega^{f}}^{2}+\left\|e_{h}^{n+1}\right\|_{0, \Omega^{f}}^{2}\right) \\
& +\sum_{n=0}^{N} C \rho^{s} \delta t\left\|\frac{e_{H}^{n+1}-e_{H}^{n}}{\delta t}\right\|_{0, \Omega^{s}}^{2}+\sum_{n=0}^{N} \frac{C h \rho^{f} \delta t}{H^{\alpha}}\left\|\frac{e_{H}^{n+1}-2 e_{H}^{n}+e_{H}^{n-1}}{\delta t}\right\|_{0, \Omega^{s}}^{2} \\
& +\sum_{n=0}^{N} C \rho^{f} \delta t\left\|\partial_{t} \boldsymbol{u}\left(t^{n+1}\right)-\frac{\boldsymbol{u}_{h}\left(t^{n+1}\right)-\boldsymbol{u}_{h}\left(t^{n}\right)}{\delta t}\right\|_{0, \Omega^{f}}^{2} \\
& +\sum_{n=0}^{N} C \rho^{s} \delta t\left\|\partial_{t t} \boldsymbol{\eta}\left(t^{n+1}\right)-\frac{\boldsymbol{\eta}_{H}\left(t^{n+1}\right)-2 \boldsymbol{\eta}_{H}\left(t^{n}\right)+\boldsymbol{\eta}_{H}\left(t^{n-1}\right)}{\delta t^{2}}\right\|_{0, \Omega^{s}}^{2}+\sum_{n=0}^{N} \mathcal{A}_{h}^{n+1} \text {. }
\end{aligned}
$$


Remark 7 We note, in particular, that the second and fourth term in the the right-hand side have been separated intentionally. Indeed, as observed in the following, the second one induces a lower convergence rate in time for the semiimplicit scheme, while the fourth one doesn't.

For the sake of clarity, in the following we will redefine $\sum_{n=0}^{N} \mathcal{A}_{h}^{n+1}$ as $\sum_{i=1}^{4} T_{i}^{N}$, where $T_{i}^{N}$ for $1 \leq i \leq 4$ are introduced and analyzed below. We set

$$
\begin{gathered}
T_{1}^{N} \stackrel{\text { def }}{=}-\sum_{n=0}^{N} a^{s}\left(\boldsymbol{\eta}_{H}\left(t^{n+1}\right)-\boldsymbol{\eta}\left(t^{n+1}\right), e_{H}^{n+1}-e_{H}^{n}\right), \\
T_{2}^{N} \stackrel{\text { def }}{=}-\sum_{n=0}^{N}\left\langle\xi\left(t^{n+1}\right), e_{H}^{n+1}-e_{H}^{n}-\pi_{h}\left(e_{H}^{n+1}-e_{H}^{n}\right)\right\rangle_{\Sigma}, \\
T_{3}^{N} \stackrel{\text { def }}{=} \sum_{n=0}^{N} 2 \mu\left(\boldsymbol{\epsilon}\left(\boldsymbol{u}\left(t^{n+1}\right)-\boldsymbol{u}_{h}\left(t^{n+1}\right)\right), \boldsymbol{\epsilon}\left(\mathcal{L}_{h}\left(e_{H}^{n+1}-e_{H}^{n}\right)\right)\right)_{\Omega^{f}},
\end{gathered}
$$

and

$$
T_{4}^{N} \stackrel{\text { def }}{=} \sum_{n=0}^{N}\left(p_{h}\left(t^{n+1}\right)-p\left(t^{n+1}\right), \nabla \cdot \mathcal{L}_{h}\left(e_{H}^{n+1}-e_{H}^{n}\right)\right)_{\Omega^{f}} .
$$
have

Considering the term $T_{1}^{N}=-\sum_{n=0}^{N} a^{s}\left(\boldsymbol{\eta}_{H}\left(t^{n+1}\right)-\boldsymbol{\eta}\left(t^{n+1}\right), e_{H}^{n+1}-e_{H}^{n}\right)$ we

$$
\begin{aligned}
T_{1}^{N}= & -\sum_{n=1}^{N+1} a^{s}\left(\boldsymbol{\eta}_{H}\left(t^{n}\right)-\boldsymbol{\eta}\left(t^{n}\right), e_{H}^{n}\right)+\sum_{n=0}^{N} a^{s}\left(\boldsymbol{\eta}_{H}\left(t^{n+1}\right)-\boldsymbol{\eta}\left(t^{n+1}\right), e_{H}^{n}\right) \\
= & \sum_{n=1}^{N} a^{s}\left(\left(\boldsymbol{\eta}_{H}\left(t^{n+1}\right)-\boldsymbol{\eta}\left(t^{n+1}\right)\right)-\left(\boldsymbol{\eta}_{H}\left(t^{n}\right)-\boldsymbol{\eta}\left(t^{n}\right)\right), e_{H}^{n}\right) \\
& -a^{s}\left(\boldsymbol{\eta}_{H}\left(t^{N+1}\right)-\boldsymbol{\eta}\left(t^{N+1}\right), e_{H}^{N+1}\right)+a^{s}\left(\boldsymbol{\eta}_{H}\left(t^{1}\right)-\boldsymbol{\eta}\left(t^{1}\right), e_{H}^{0}\right)
\end{aligned}
$$

Choosing $\boldsymbol{\eta}_{H}\left(t^{0}\right)=\boldsymbol{\eta}_{H}^{0}\left(\right.$ i.e. $\left.e_{H}^{0}=0\right)$ and using the continuity of $a^{s}(\cdot, \cdot)$ we get

$$
\begin{aligned}
\left|T_{1}^{N}\right| \leq & \sum_{n=1}^{N} C \delta t\left(\left\|\frac{\boldsymbol{\eta}_{H}\left(t^{n+1}\right)-\boldsymbol{\eta}\left(t^{n+1}\right)}{\delta t}-\frac{\boldsymbol{\eta}_{H}\left(t^{n}\right)-\boldsymbol{\eta}\left(t^{n}\right)}{\delta t}\right\|_{V^{s}}^{2}+a^{s}\left(e_{H}^{n}, e_{H}^{n}\right)\right) \\
& +C\left\|\boldsymbol{\eta}_{H}\left(t^{N+1}\right)-\boldsymbol{\eta}\left(t^{N+1}\right)\right\|_{V^{s}}^{2}+\frac{\gamma_{4}}{2} a^{s}\left(e_{H}^{N+1}, e_{H}^{N+1}\right) .
\end{aligned}
$$

By a Taylor expansion, since we assume that the continuous solution is smooth enough, $T_{1}^{N}$ can be bounded by

$$
\begin{aligned}
\left|T_{1}^{N}\right| \leq & \sum_{n=1}^{N} C \delta t\left(\left\|\partial_{t} \boldsymbol{\eta}\left(t^{n+1}\right)-\frac{\boldsymbol{\eta}_{H}\left(t^{n+1}\right)-\boldsymbol{\eta}_{H}\left(t^{n}\right)}{\delta t}\right\|_{V^{s}}^{2}+a^{s}\left(e_{H}^{n}, e_{H}^{n}\right)\right) \\
& +C\left\|\boldsymbol{\eta}_{H}\left(t^{N+1}\right)-\boldsymbol{\eta}\left(t^{N+1}\right)\right\|_{V^{s}}^{2}+\frac{\gamma_{4}}{2} a^{s}\left(e_{H}^{N+1}, e_{H}^{N+1}\right)+C \delta t^{2} .
\end{aligned}
$$

Before going on, we observe that the quantity $\left\|\boldsymbol{\eta}_{H}\left(t^{N+1}\right)-\boldsymbol{\eta}\left(t^{N+1}\right)\right\|_{V^{s}}^{2}$ will be estimated by means of estimate (36). Moreover, the Taylor formula and the fact that $e_{H}^{0}=0$ will be used also in the remaining estimates of $T_{i}^{N}, \forall 2 \leq i \leq 4$, but their notification will be omitted. 
The term $T_{2}^{N}=-\sum_{n=0}^{N}\left\langle\xi\left(t^{n+1}\right), e_{H}^{n+1}-e_{H}^{n}-\pi_{h}\left(e_{H}^{n+1}-e_{H}^{n}\right)\right\rangle_{\Sigma}$, representing the consistency error associated with the non-conforming matching at the interface, can be rewritten as

$$
\begin{aligned}
T_{2}^{N}= & -\sum_{n=1}^{N+1}\left\langle\xi\left(t^{n}\right), e_{H}^{n}-\pi_{h}\left(e_{H}^{n}\right)\right\rangle_{\Sigma}+\sum_{n=0}^{N}\left\langle\xi\left(t^{n+1}\right), e_{H}^{n}-\pi_{h}\left(e_{H}^{n}\right)\right\rangle_{\Sigma} \\
= & -\sum_{n=1}^{N}\left\langle\xi\left(t^{n+1}\right)-\xi\left(t^{n}\right), e_{H}^{n}-\pi_{h}\left(e_{H}^{n}\right)\right\rangle_{\Sigma} \\
& -\left\langle\xi\left(t^{N+1}\right), e_{H}^{N+1}-\pi_{h}\left(e_{H}^{N+1}\right)\right\rangle_{\Sigma}+\left\langle\xi\left(t^{1}\right), e_{H}^{0}-\pi_{h}\left(e_{H}^{0}\right)\right\rangle_{\Sigma} .
\end{aligned}
$$

Depending on the choice of the operator $\pi_{h}$ we have different kind of estimates. If $\pi_{h}$ is a mortar-type operator, using property (23) we have

$$
\begin{aligned}
T_{2}^{N}= & -\sum_{n=1}^{N} \delta t\left\langle\frac{\xi\left(t^{n+1}\right)-\tilde{\pi}_{h}\left(\xi\left(t^{n+1}\right)\right)}{\delta t}-\frac{\xi\left(t^{n}\right)-\tilde{\pi}_{h}\left(\xi\left(t^{n}\right)\right)}{\delta t}, e_{H}^{n}-\pi_{h}\left(e_{H}^{n}\right)\right\rangle_{\Sigma} \\
& -\left\langle\xi\left(t^{N+1}\right)-\tilde{\pi}_{h}\left(\xi\left(t^{N+1}\right)\right), e_{H}^{N+1}-\pi_{h}\left(e_{H}^{N+1}\right)\right\rangle_{\Sigma}+\left\langle\xi\left(t^{1}\right)-\tilde{\pi}_{h}\left(\xi\left(t^{1}\right)\right), e_{H}^{0}-\pi_{h}\left(e_{H}^{0}\right)\right\rangle_{\Sigma},
\end{aligned}
$$

where $\tilde{\pi}_{h}\left(\xi\left(t^{i}\right)\right) \in \tilde{V}_{h}^{f}(\Sigma)$ denotes the $L^{2}$ orthogonal projection of $\xi\left(t^{i}\right)$ on $V_{h}^{f}(\Sigma)$.

First we estimate $\left|T_{2}^{N}\right|$ as

$$
\begin{aligned}
\left|T_{2}^{N}\right| \leq & \sum_{n=1}^{N} C \delta t\left(\left\|\left(\mathcal{I}-\tilde{\pi}_{h}\right)\left(\frac{\xi\left(t^{n+1}\right)-\xi\left(t^{n}\right)}{\delta t}\right)\right\|_{\left(H_{00}^{\frac{1}{2}}(\Sigma)\right)^{\prime}}^{2}+\left\|e_{H}^{n}-\pi_{h}\left(e_{H}^{n}\right)\right\|_{H_{00}^{\frac{1}{2}}(\Sigma)}^{2}\right) \\
& +C\left\|\left(\mathcal{I}-\tilde{\pi}_{h}\right)\left(\xi\left(t^{N+1}\right)\right)\right\|_{\left(H_{00}^{\frac{1}{2}}(\Sigma)\right)^{\prime}}^{2}+\frac{\gamma_{4}}{2}\left\|e_{H}^{N+1}-\pi_{h}\left(e_{H}^{N+1}\right)\right\|_{H_{00}^{\frac{1}{2}}(\Sigma)}^{2} .
\end{aligned}
$$

Then, the $H_{00}^{\frac{1}{2}}(\Sigma)$-stability of $\pi_{h}$, see $(27)$, and the coercivity of $a_{s}(\cdot, \cdot)$ on $H_{0, \Gamma_{d}^{s}}^{1}\left(\Omega^{s}\right)$ yield

$$
\left\|e_{H}^{n}-\pi_{h}\left(e_{H}^{n}\right)\right\|_{H_{00}^{\frac{1}{2}}(\Sigma)}^{2} \leq C\left\|e_{H}^{n}\right\|_{H_{00}^{\frac{1}{2}}(\Sigma)}^{2} \leq C\left\|e_{H}^{n}\right\|_{1, \Omega^{s}}^{2} \leq a_{s}\left(e_{H}^{n}, e_{H}^{n}\right) .
$$

Therefore $\left|T_{2}^{N}\right|$ can be controlled by

$$
\begin{aligned}
\left|T_{2}^{N}\right| \leq & \sum_{n=1}^{N} C \delta t\left(\left\|\left(\mathcal{I}-\tilde{\pi}_{h}\right)\left(\frac{\xi\left(t^{n+1}\right)-\xi\left(t^{n}\right)}{\delta t}\right)\right\|_{\left(H_{00}^{\frac{1}{2}}(\Sigma)\right)^{\prime}}^{2}+a^{s}\left(e_{H}^{n}, e_{H}^{n}\right)\right) \\
& +C\left\|\left(\mathcal{I}-\tilde{\pi}_{h}\right)\left(\xi\left(t^{N+1}\right)\right)\right\|_{\left(H_{00}^{\frac{1}{2}}(\Sigma)\right)^{\prime}}^{2}+\frac{\gamma_{4}}{2} a^{s}\left(e_{H}^{N+1}, e_{H}^{N+1}\right) \\
\leq & \sum_{n=1}^{N} C \delta t\left(\left\|\left(\mathcal{I}-\tilde{\pi}_{h}\right)\left(\partial_{t} \xi\left(t^{n+1}\right)\right)\right\|_{\left(H_{00}^{\frac{1}{2}}(\Sigma)\right)^{\prime}}^{2}+a^{s}\left(e_{H}^{n}, e_{H}^{n}\right)\right) \\
& +C\left\|\left(\mathcal{I}-\tilde{\pi}_{h}\right)\left(\xi\left(t^{N+1}\right)\right)\right\|_{\left(H_{00}^{\frac{1}{2}}(\Sigma)\right)^{\prime}}^{2}+\frac{\gamma_{4}}{2} a^{s}\left(e_{H}^{N+1}, e_{H}^{N+1}\right)+C \delta t^{2} .(68)
\end{aligned}
$$

To estimate the quantity $\left\|\left(\mathcal{I}-\tilde{\pi}_{h}\right)\left(\xi\left(t^{N+1}\right)\right)\right\|_{\left(H_{00}^{\frac{1}{2}}(\Sigma)\right)^{\prime}}\left(\right.$ as well as $\left.\left\|\left(\mathcal{I}-\tilde{\pi}_{h}\right)\left(\partial_{t} \xi\left(t^{n+1}\right)\right)\right\|_{\left(H_{00}^{\frac{1}{2}}(\Sigma)\right)^{\prime}}\right)$ we make use of (24) and of the standard finite element estimate on $\left\{\boldsymbol{v}_{h} \in\right.$ $\mathcal{C}^{0}(\bar{\Sigma}), \boldsymbol{v}_{h \mid K} \in \mathbb{P}_{k-1}, \forall K \in \mathcal{T}_{h}^{f}$ with $\left.|K \cap \Sigma| \neq 0\right\} \subset \tilde{V}_{h}^{f}(\Sigma)$,

$$
\begin{gathered}
\quad\left\|\left(\mathcal{I}-\tilde{\pi}_{h}\right)\left(\xi\left(t^{i}\right)\right)\right\|_{\left(H_{00}^{\frac{1}{2}}(\Sigma)\right)^{\prime}}=\left\|\xi\left(t^{i}\right)-\tilde{\xi}_{h}\left(t^{i}\right)-\tilde{\pi}_{h}\left(\xi\left(t^{i}\right)-\tilde{\xi}_{h}\left(t^{i}\right)\right)\right\|_{\left(H_{00}^{\frac{1}{2}}(\Sigma)\right)^{\prime}} \\
\leq C h^{\frac{1}{2}}\left\|\xi\left(t^{i}\right)-\tilde{\xi}_{h}\left(t^{i}\right)\right\|_{0, \Sigma} \leq C h^{l}\left\|\xi\left(t^{i}\right)\right\|_{l-\frac{1}{2}, \Sigma}, \quad \forall \frac{1}{2} \leq l \leq k, \quad \forall \tilde{\xi}_{h} \in \tilde{V}_{h}^{f}(\Sigma) .
\end{gathered}
$$


We note, in particular, that $\tilde{V}_{h}^{f}(\Sigma)$ has good approximations properties for $\left(H_{00}^{\frac{1}{2}}(\Sigma)\right)^{\prime}$. Therefore, in the mortar case, $T_{2}^{N}$ can be controlled by

$$
\left|T_{2}^{N}\right| \leq \sum_{n=1}^{N} C \delta t a^{s}\left(e_{H}^{n}, e_{H}^{n}\right)+\frac{\gamma_{4}}{2} a^{s}\left(e_{H}^{N+1}, e_{H}^{N+1}\right)+C \delta t^{2}+C h^{2 l}, \quad \forall \frac{1}{2} \leq l \leq k .
$$

Whereas, when $\pi_{h}$ is a finite element interpolation operator, we can conclude that

$$
\begin{aligned}
\left|T_{2}^{N}\right| & \leq \sum_{n=1}^{N} C \delta t\left\|e_{H}^{n}-\pi_{h}\left(e_{H}^{n}\right)\right\|_{0, \Sigma}+C\left\|e_{H}^{N+1}-\pi_{h}\left(e_{H}^{N+1}\right)\right\|_{0, \Sigma} \\
& \leq \sum_{n=1}^{N} C \delta t h^{l}\left\|e_{H}^{n}\right\|_{l, \Sigma}+C h^{l}\left\|e_{H}^{N+1}\right\|_{l, \Sigma} \\
& \leq \sum_{n=1}^{N} C \delta t\left\|e_{H}^{n}\right\|_{l, \Sigma}^{2}+C\left\|e_{H}^{N+1}\right\|_{l, \Sigma}^{2}+C h^{2 l}, \quad \text { with } \quad l \leq \min \left(r-\frac{\alpha}{2}, k+1\right)
\end{aligned}
$$

where we have used the approximation property (21). Finally, the inequality

$$
\|\boldsymbol{v}\|_{l, \Sigma} \leq C\|\boldsymbol{v}\|_{l+\frac{\alpha}{2}, \Omega^{s}} \leq C\|\boldsymbol{v}\|_{r, \Omega^{s}}, \forall \boldsymbol{v} \in H^{r}\left(\Omega^{s}\right),
$$

and the coercivity of $a^{s}(\cdot, \cdot)$ in $V^{s} \subset H^{r}\left(\Omega^{s}\right)$ lead to

$$
\left|T_{2}^{N}\right| \leq \sum_{n=1}^{N} C \delta t a^{s}\left(e_{H}^{n}, e_{H}^{n}\right)+\frac{\gamma_{4}}{2} a^{s}\left(e_{H}^{N+1}, e_{H}^{N+1}\right)+C h^{2 l}, \quad \text { with } l \leq \min \left(r-\frac{\alpha}{2}, k+1\right) .
$$

Like the previous terms, $T_{3}^{N}=\sum_{n=0}^{N} 2 \mu\left(\boldsymbol{\epsilon}\left(\boldsymbol{u}\left(t^{n+1}\right)-\boldsymbol{u}_{h}\left(t^{n+1}\right)\right), \boldsymbol{\epsilon}\left(\mathcal{L}_{h}\left(e_{H}^{n+1}-\right.\right.\right.$ $\left.\left.\left.e_{H}^{n}\right)\right)\right)_{\Omega^{f}}$ is first rewritten as

$$
\begin{aligned}
T_{3}^{N}= & \sum_{n=1}^{N+1} 2 \mu\left(\boldsymbol{\epsilon}\left(\boldsymbol{u}\left(t^{n}\right)-\boldsymbol{u}_{h}\left(t^{n}\right)\right), \boldsymbol{\epsilon}\left(\mathcal{L}_{h}\left(e_{H}^{n}\right)\right)\right)_{\Omega^{f}}-\sum_{n=0}^{N} 2 \mu\left(\boldsymbol{\epsilon}\left(\boldsymbol{u}\left(t^{n+1}\right)-\boldsymbol{u}_{h}\left(t^{n+1}\right)\right), \boldsymbol{\epsilon}\left(\mathcal{L}_{h}\left(e_{H}^{n}\right)\right)\right)_{\Omega^{f}} \\
= & \sum_{n=1}^{N} 2 \mu \delta t\left(\boldsymbol{\epsilon}\left(\frac{\boldsymbol{u}\left(t^{n+1}\right)-\boldsymbol{u}\left(t^{n}\right)}{\delta t}\right)-\boldsymbol{\epsilon}\left(\frac{\boldsymbol{u}_{h}\left(t^{n+1}\right)-\boldsymbol{u}_{h}\left(t^{n}\right)}{\delta t}\right), \boldsymbol{\epsilon}\left(\mathcal{L}_{h}\left(e_{H}^{n}\right)\right)\right)_{\Omega^{f}} \\
& +2 \mu\left(\boldsymbol{\epsilon}\left(\boldsymbol{u}\left(t^{N+1}\right)-\boldsymbol{u}_{h}\left(t^{N+1}\right)\right), \boldsymbol{\epsilon}\left(\mathcal{L}_{h}\left(e_{H}^{N+1}\right)\right)\right)_{\Omega^{f}}-2 \mu\left(\boldsymbol{\epsilon}\left(\boldsymbol{u}\left(t^{1}\right)-\boldsymbol{u}_{h}\left(t^{1}\right)\right), \boldsymbol{\epsilon}\left(\mathcal{L}_{h}\left(e_{H}^{0}\right)\right)\right)_{\Omega^{f}},
\end{aligned}
$$

and then bounded by

$$
\begin{aligned}
\left|T_{3}^{N}\right| \leq & \sum_{n=1}^{N} C \delta t\left(\mu^{2}\left\|\boldsymbol{\epsilon}\left(\partial_{t} \boldsymbol{u}\left(t^{n+1}\right)-\frac{\boldsymbol{u}_{h}\left(t^{n+1}\right)-\boldsymbol{u}_{h}\left(t^{n}\right)}{\delta t}\right)\right\|_{0, \Omega^{f}}^{2}+\left\|\boldsymbol{\epsilon}\left(\mathcal{L}_{h}\left(e_{H}^{n}\right)\right)\right\|_{0, \Omega^{f}}^{2}\right) \\
& +C \mu^{2}\left\|\boldsymbol{\epsilon}\left(\boldsymbol{u}\left(t^{N+1}\right)-\boldsymbol{u}_{h}\left(t^{N+1}\right)\right)\right\|_{0, \Omega^{f}}^{2}+C\left\|\boldsymbol{\epsilon}\left(\mathcal{L}_{h}\left(e_{H}^{N+1}\right)\right)\right\|_{0, \Omega^{f}}^{2}+C \delta t^{2} .
\end{aligned}
$$

The definition of $\mathcal{L}_{h}$, the inequality (44) and the stability properties of $\pi_{h}$ (see 20 in the finite element interpolation operator case or 27 in the mortar case) yield$$
\left\|\boldsymbol{\epsilon}\left(\mathcal{L}_{h}\left(e_{H}^{n}\right)\right)\right\|_{0, \Omega^{f}}^{2} \leq C\left\|\pi_{h}\left(e_{H}^{n}\right)\right\|_{H_{00}^{\frac{1}{2}}(\Sigma)}^{2} \leq C\left\|e_{H}^{n}\right\|_{H_{00}^{\frac{1}{2}}(\Sigma)}^{2} \leq C a^{s}\left(e_{H}^{n}, e_{H}^{n}\right) \quad \forall n \geq 0,
$$ 
that gives

$$
\begin{aligned}
\left|T_{3}^{N}\right| \leq & \sum_{n=1}^{N} C \delta t\left(\mu^{2}\left\|\boldsymbol{\epsilon}\left(\partial_{t} \boldsymbol{u}\left(t^{n+1}\right)-\frac{\boldsymbol{u}_{h}\left(t^{n+1}\right)-\boldsymbol{u}_{h}\left(t^{n}\right)}{\delta t}\right)\right\|_{0, \Omega^{f}}^{2}+a^{s}\left(e_{H}^{n}, e_{H}^{n}\right)\right) \\
& +C \mu^{2}\left\|\boldsymbol{u}\left(t^{N+1}\right)-\boldsymbol{u}_{h}\left(t^{N+1}\right)\right\|_{1, \Omega^{f}}^{2}+\frac{\gamma_{4}}{2} a^{s}\left(e_{H}^{N+1}, e_{H}^{N+1}\right)+C \delta t^{2} .
\end{aligned}
$$

Note that here $\left\|\boldsymbol{u}\left(t^{N+1}\right)-\boldsymbol{u}_{h}\left(t^{N+1}\right)\right\|_{1, \Omega^{f}}^{2}$ can be estimated as in 38. that

Finally, for $T_{4}^{N}=\sum_{n=0}^{N}\left(p_{h}\left(t^{n+1}\right)-p\left(t^{n+1}\right), \nabla \cdot \mathcal{L}_{h}\left(e_{H}^{n+1}-e_{H}^{n}\right)\right)_{\Omega^{f}}$ we have

$$
\begin{aligned}
T_{4}^{N} \stackrel{\text { def }}{=} & \sum_{n=1}^{N+1}\left(p_{h}\left(t^{n}\right)-p\left(t^{n}\right), \boldsymbol{\nabla} \cdot \mathcal{L}_{h}\left(e_{H}^{n}\right)\right)_{\Omega^{f}}-\sum_{n=0}^{N+1}\left(p_{h}\left(t^{n+1}\right)-p\left(t^{n+1}\right), \boldsymbol{\nabla} \cdot \mathcal{L}_{h}\left(e_{H}^{n}\right)\right)_{\Omega^{f}} \\
= & \sum_{n=1}^{N} \delta t\left(\left(\frac{p_{h}\left(t^{n+1}\right)-p_{h}\left(t^{n}\right)}{\delta t}\right)-\left(\frac{p\left(t^{n+1}\right)-p\left(t^{n}\right)}{\delta t}\right), \boldsymbol{\nabla} \cdot\left(\mathcal{L}_{h}\left(e_{H}^{n}\right)\right)\right)_{\Omega^{f}} \\
& \left.+\left(p_{h}\left(t^{N+1}\right)-p\left(t^{N+1}\right), \boldsymbol{\nabla} \cdot\left(\mathcal{L}_{h}\left(e_{H}^{N+1}\right)\right)\right)_{\Omega^{f}}-\left(p_{h}\left(t^{1}\right)-p\left(t^{1}\right)\right), \boldsymbol{\nabla} \cdot\left(\mathcal{L}_{h}\left(e_{H}^{0}\right)\right)\right)_{\Omega^{f}}
\end{aligned}
$$

and therefore

$$
\begin{aligned}
\left|T_{4}^{N}\right| \leq & \sum_{n=1}^{N} C \delta t\left(\left\|\partial_{t} p\left(t^{n+1}\right)-\frac{p_{h}\left(t^{n+1}\right)-p_{h}\left(t^{n}\right)}{\delta t}\right\|_{0, \Omega^{f}}^{2}+a^{s}\left(e_{H}^{n}, e_{H}^{n}\right)\right) \\
& +C\left\|p\left(t^{N+1}\right)-p_{h}\left(t^{N+1}\right)\right\|_{0, \Omega^{f}}^{2}+\frac{\gamma_{4}}{2} a^{s}\left(e_{H}^{N+1}, e_{H}^{N+1}\right)+C \delta t^{2},
\end{aligned}
$$

where, as for $T_{3}^{N}$, we have used the fact that

$$
\left\|\nabla \cdot\left(\mathcal{L}_{h}\left(e_{H}^{n}\right)\right)\right\|_{0, \Omega^{f}}^{2} \leq C a^{s}\left(e_{H}^{n}, e_{H}^{n}\right) \quad \forall n \geq 0 .
$$

Note moreover that the quantity $\left\|p\left(t^{N+1}\right)-p_{h}\left(t^{N+1}\right)\right\|_{0, \Omega^{f}}^{2}$ verifies estimate (39).

Part 4. We finally replace in 66 the terms $T_{i}^{N}$, for $1 \leq i \leq 4$, by the corresponding bounds (67), (69) (or (70) depending on the type of matching operator at the interface), 72 and $(73)$, and we set $\gamma_{1}=\frac{1}{4}, \gamma_{2}=\gamma_{3}=\frac{1}{2}$ and 
$\gamma_{4}=\frac{1}{8}$. After some rearrangements, we obtain

$$
\begin{aligned}
E^{N+1}+ & \frac{\rho^{f}}{4} \sum_{n=0}^{N}\left[\left\|\tilde{e}_{h}^{n+1}-e_{h}^{n}\right\|_{0, \Omega^{f}}^{2}+\left\|e_{h}^{n+1}-\tilde{e}_{h}^{n+1}\right\|_{0, \Omega^{f}}^{2}\right] \\
+ & \sum_{n=0}^{N} \mu \delta t\left\|\boldsymbol{\epsilon}\left(\tilde{e}_{h}^{n+1}\right)\right\|_{0, \Omega^{f}}^{2}+\frac{1}{2} \sum_{n=0}^{N} a^{s}\left(e_{H}^{n+1}-e_{H}^{n}, e_{H}^{n+1}-e_{H}^{n}\right) \\
+ & \sum_{n=0}^{N}\left(\frac{\rho^{s}}{2}-\frac{C h \rho^{f}}{H^{\alpha}}-\frac{C \mu \delta t}{h H^{\alpha}}\right)\left\|\frac{e_{H}^{n+1}-2 e_{H}^{n}+e_{H}^{n-1}}{\delta t}\right\|_{0, \Omega^{s}}^{2} \\
\leq & \sum_{n=0}^{N} C \rho^{f} \delta t\left(\left\|\tilde{e}_{h}^{n+1}-e_{h}^{n+1}\right\|_{0, \Omega^{f}}^{2}+\left\|e_{h}^{n+1}\right\|_{0, \Omega^{f}}^{2}\right) \\
& +\sum_{n=0}^{N} C \rho^{s} \delta t\left\|\frac{e_{H}^{n+1}-e_{H}^{n}}{\delta t}\right\|_{0, \Omega^{s}}^{2}+\sum_{n=0}^{N} \frac{C h \rho^{f} \delta t}{H^{\alpha}}\left\|\frac{e_{H}^{n+1}-2 e_{H}^{n}+e_{H}^{n-1}}{\delta t}\right\|_{0, \Omega^{s}}^{2} \\
& +\sum_{n=1}^{N} C \delta t a^{s}\left(e_{H}^{n}, e_{N}^{n}\right)+\mathcal{I}+\mathcal{H}+\mathcal{T}+C \delta t^{2}+C h^{2 l},
\end{aligned}
$$

where

$$
E^{n+1} \stackrel{\text { def }}{=} \frac{\rho^{f}}{2}\left\|e_{h}^{n+1}\right\|_{0, \Omega^{f}}^{2}+\frac{\rho^{s}}{2}\left\|\frac{e_{H}^{n+1}-e_{H}^{n}}{\delta t}\right\|_{0, \Omega^{s}}^{2}+\frac{1}{4} a^{s}\left(e_{H}^{n+1}, e_{H}^{n+1}\right) .
$$

Here, $\mathcal{T}$ contains the terms that cause a reduction of the convergence rate in time, $\mathcal{H}$ the ones that introduce a time consistency error, while $\mathcal{I}$ regroups the remaining terms, associated with the space discretization errors. More precisely

$\mathcal{I} \stackrel{\text { def }}{=} C\left\|\boldsymbol{\eta}_{H}\left(t^{N+1}\right)-\boldsymbol{\eta}\left(t^{N+1}\right)\right\|_{V^{s}}^{2}+C \mu^{2}\left\|\boldsymbol{u}\left(t^{N+1}\right)-\boldsymbol{u}_{h}\left(t^{N+1}\right)\right\|_{1, \Omega^{f}}^{2}+C\left\|p\left(t^{N+1}\right)-p_{h}\left(t^{N+1}\right)\right\|_{0, \Omega^{f}}^{2}$.

Using (36), (38) and 39, , $\mathcal{I}$ can be bounded by

$$
\mathcal{I} \leq C \delta t^{2}+C h^{2 k}+C H^{2 m}+C h^{2 l} .
$$

In (75), $k$ and $m$ represent (and will represent in all that follows) the finite element order respectively for the fluid and the structure part, while $l \leq$ $\min \left(r-\frac{\alpha}{2}, k+1\right)$ (resp. $\left.\frac{1}{2} \leq l \leq k+1\right)$ for a finite element interpolation (resp. mortar) matching operator at the interface. 
$\mathcal{H}$ regroups some of the terms coming from the estimates of $\mathcal{D}_{h}^{n+1}$ and of $T_{1}^{N}, T_{3}^{N}, T_{4}^{N}$, and is equal to

$$
\begin{aligned}
\mathcal{H} \stackrel{\text { def }}{=} & \sum_{n=0}^{N} C \frac{h \rho^{f} \delta t}{H^{\alpha}}\left\|\frac{\boldsymbol{\eta}_{H}\left(t^{n+1}\right)-2 \boldsymbol{\eta}_{H}\left(t^{n}\right)+\boldsymbol{\eta}_{H}\left(t^{n-1}\right)}{\delta t}\right\|_{0, \Omega^{s}}^{2} \\
& +\sum_{n=0}^{N} C \rho^{f} \delta t\left\|\partial_{t} \boldsymbol{u}\left(t^{n+1}\right)-\frac{\boldsymbol{u}_{h}\left(t^{n+1}\right)-\boldsymbol{u}_{h}\left(t^{n}\right)}{\delta t}\right\|_{0, \Omega^{f}}^{2} \\
& +\sum_{n=0}^{N} C \rho^{s} \delta t\left\|\partial_{t t} \boldsymbol{\eta}\left(t^{n+1}\right)-\frac{\boldsymbol{\eta}_{H}\left(t^{n+1}\right)-2 \boldsymbol{\eta}_{H}\left(t^{n}\right)+\boldsymbol{\eta}_{H}\left(t^{n-1}\right)}{\delta t^{2}}\right\|_{0, \Omega^{s}}^{2} \\
& +\sum_{n=1}^{N} C \delta t\left\|\partial_{t} \boldsymbol{\eta}\left(t^{n+1}\right)-\frac{\boldsymbol{\eta}_{H}\left(t^{n+1}\right)-\boldsymbol{\eta}_{H}\left(t^{n}\right)}{\delta t}\right\|_{V^{s}}^{2} \\
& +\sum_{n=1}^{N} C \delta t \mu^{2}\left\|\boldsymbol{\epsilon}\left(\partial_{t} \boldsymbol{u}\left(t^{n+1}\right)-\frac{\boldsymbol{u}_{h}\left(t^{n+1}\right)-\boldsymbol{u}_{h}\left(t^{n}\right)}{\delta t}\right)\right\|_{0, \Omega^{f}}^{2} \\
& +\sum_{n=1}^{N} C \delta t\left\|\partial_{t} p\left(t^{n+1}\right)-\frac{p_{h}\left(t^{n+1}\right)-p_{h}\left(t^{n}\right)}{\delta t}\right\|_{0, \Omega^{f}}^{2} \cdot
\end{aligned}
$$

The first term can be bounded by

$$
\sum_{n=0}^{N} C \frac{h \rho^{f} \delta t}{H^{\alpha}}\left\|\frac{\boldsymbol{\eta}_{H}\left(t^{n+1}\right)-2 \boldsymbol{\eta}_{H}\left(t^{n}\right)+\boldsymbol{\eta}_{H}\left(t^{n-1}\right)}{\delta t}\right\|_{0, \Omega^{s}}^{2} \leq C \frac{h \rho^{f}}{H^{\alpha}} \delta t^{2},
$$

using the fact that

$$
\begin{aligned}
& \left\|\frac{\boldsymbol{\eta}_{H}\left(t^{n+1}\right)-2 \boldsymbol{\eta}_{H}\left(t^{n}\right)+\boldsymbol{\eta}_{H}\left(t^{n-1}\right)}{\delta t}\right\|_{0, \Omega^{s}}^{2} \\
& \quad \leq C\left(\left\|\frac{\boldsymbol{\eta}_{H}\left(t^{n+1}\right)-\boldsymbol{\eta}_{H}\left(t^{n}\right)}{\delta t}-\partial_{t} \boldsymbol{\eta}_{H}\left(t^{n}\right)\right\|_{0, \Omega^{s}}^{2}+\left\|\partial_{t} \boldsymbol{\eta}_{H}\left(t^{n}\right)-\frac{\boldsymbol{\eta}_{H}\left(t^{n}\right)-\boldsymbol{\eta}_{H}\left(t^{n-1}\right)}{\delta t}\right\|_{0, \Omega^{s}}^{2}\right) \\
& \quad \leq C \delta t^{2} .
\end{aligned}
$$

In order to control the remaining time consistency errors, we will follow the same steps as in the derivation of (36), 38 and 39 and use the definitions of the different correction terms $\left(\boldsymbol{z}_{h}, \mu_{h}, \boldsymbol{c}_{H}\right)$. For example, considering

$$
\begin{aligned}
& \left\|\partial_{t} \boldsymbol{u}\left(t^{n+1}\right)-\frac{\boldsymbol{u}_{h}\left(t^{n+1}\right)-\boldsymbol{u}_{h}\left(t^{n}\right)}{\delta t}\right\|_{0, \Omega^{f}}^{2} \text { we have } \\
& \left\|\partial_{t} \boldsymbol{u}\left(t^{n+1}\right)-\frac{\boldsymbol{u}_{h}\left(t^{n+1}\right)-\boldsymbol{u}_{h}\left(t^{n}\right)}{\delta t}\right\|_{0, \Omega^{f}}^{2} \\
& \quad=\left\|\partial_{t} \boldsymbol{u}\left(t^{n+1}\right)-\frac{P_{h}^{f}(\boldsymbol{u})\left(t^{n+1}\right)-P_{h}^{f}(\boldsymbol{u})\left(t^{n}\right)}{\delta t}-\frac{\boldsymbol{z}_{h}\left(t^{n+1}\right)-\boldsymbol{z}_{h}\left(t^{n}\right)}{\delta t}\right\|_{0, \Omega^{f}}^{2} \\
& \leq C\left\|\partial_{t} \boldsymbol{u}\left(t^{n+1}\right)-P_{h}^{f}\left(\partial_{t} \boldsymbol{u}\right)\left(t^{n+1}\right)\right\|_{0, \Omega^{f}}^{2}+C\left\|\frac{\boldsymbol{z}_{h}\left(t^{n+1}\right)-\boldsymbol{z}_{h}\left(t^{n}\right)}{\delta t}\right\|_{0, \Omega^{f}}^{2}+C \delta t^{2} .
\end{aligned}
$$


The first term can be estimated by remembering the definition of $P_{h}^{f}$ :

$$
\left\|\partial_{t} \boldsymbol{u}\left(t^{n+1}\right)-P_{h}^{f}\left(\partial_{t} \boldsymbol{u}\right)\left(t^{n+1}\right)\right\|_{0, \Omega^{f}}^{2} \leq C \inf _{\boldsymbol{v}_{h} \in V_{h}^{f}}\left\|\partial_{t} \boldsymbol{u}\left(t^{n+1}\right)-\boldsymbol{v}_{h}\right\|_{V^{f}}^{2} .
$$

For the second term, recalling the definition of the correction term $\boldsymbol{z}_{h}$, it is easy to see that

$$
\begin{aligned}
& \left\|\frac{\boldsymbol{z}_{h}\left(t^{n+1}\right)-\boldsymbol{z}_{h}\left(t^{n}\right)}{\delta t}\right\|_{0, \Omega^{f}}^{2} \\
\leq & C\left\|\pi_{h}\left(\frac{\boldsymbol{\eta}_{H}\left(t^{n+1}\right)-2 \boldsymbol{\eta}_{H}\left(t^{n}\right)+\boldsymbol{\eta}_{H}\left(t^{n-1}\right)}{\delta t^{2}}\right)-P_{h}^{f}\left(\frac{\boldsymbol{u}\left(t^{n+1}\right)-\boldsymbol{u}\left(t^{n}\right)}{\delta t}\right)\right\|_{H_{00}^{\frac{1}{2}}(\Sigma)}^{2} .
\end{aligned}
$$

Thus the $H_{00}^{\frac{1}{2}}$-stability of $\pi_{h}$ and the fact that $\pi_{h}\left(\boldsymbol{v}_{h}\right)=\boldsymbol{v}_{h}, \forall \boldsymbol{v}_{h} \in V_{h}^{f}(\Sigma)$, lead to

$$
\begin{aligned}
& \left\|\frac{\boldsymbol{z}_{h}\left(t^{n+1}\right)-\boldsymbol{z}_{h}\left(t^{n}\right)}{\delta t}\right\|_{0, \Omega^{f}}^{2} \\
& \leq C\left\|\frac{\boldsymbol{\eta}_{H}\left(t^{n+1}\right)-2 \boldsymbol{\eta}_{H}\left(t^{n}\right)+\boldsymbol{\eta}_{H}\left(t^{n-1}\right)}{\delta t^{2}}-P_{h}^{f}\left(\frac{\boldsymbol{u}\left(t^{n+1}\right)-\boldsymbol{u}\left(t^{n}\right)}{\delta t}\right)\right\|_{H_{00}^{\frac{1}{2}}(\Sigma)}^{2} .
\end{aligned}
$$

The upper bound is now estimated following the same arguments used for the estimate of $\boldsymbol{z}_{h}$. In particular, by taking into account the fact that $\partial_{t} \boldsymbol{u}(t)=$ $\partial_{t t} \boldsymbol{\eta}(t)$ at the interface, we get

$$
\begin{aligned}
\left\|\frac{\boldsymbol{z}_{h}\left(t^{n+1}\right)-\boldsymbol{z}_{h}\left(t^{n}\right)}{\delta t}\right\|_{0, \Omega^{f}}^{2} & \leq C\left(\delta t^{2}\|\boldsymbol{\eta}\|_{W^{3, \infty}\left(0, T ; V^{s}\right)}^{2}+\inf _{\boldsymbol{v}_{H} \in V_{H}^{s}}\left\|\partial_{t t} \boldsymbol{\eta}\left(t^{i}\right)-\boldsymbol{v}_{H}\right\|_{V^{s}}^{2}\right. \\
& \left.+\inf _{\boldsymbol{v}_{h} \in V_{h}^{f}}\left\|\partial_{t} \boldsymbol{u}\left(t^{i}\right)-\boldsymbol{v}_{h}\right\|_{V^{f}}^{2}+C h^{2 l}\left\|\partial_{t t} \boldsymbol{\eta}\left(t^{i}\right)\right\|_{V^{s}}^{2}\right),
\end{aligned}
$$

with $\frac{1}{2} \leq l \leq k+1$ for the mortar case and $l \leq \min \left(r-\frac{\alpha}{2}, k+1\right)$ for the interpolation case. Therefore, with our particular choice of the finite element approximation spaces, the quantity $\left\|\partial_{t} \boldsymbol{u}\left(t^{n+1}\right)-\frac{\boldsymbol{u}_{h}\left(t^{n+1}\right)-\boldsymbol{u}_{h}\left(t^{n}\right)}{\delta t}\right\|_{0, \Omega^{f}}^{2}$ is controlled by

$$
\begin{aligned}
\left\|\partial_{t} \boldsymbol{u}\left(t^{n+1}\right)-\frac{\boldsymbol{u}_{h}\left(t^{n+1}\right)-\boldsymbol{u}_{h}\left(t^{n}\right)}{\delta t}\right\|_{0, \Omega^{f}}^{2} \leq & C\left(\delta t^{2}\|\boldsymbol{\eta}\|_{W^{3, \infty}\left(0, T ; V^{s}\right)}^{2}+\inf _{\boldsymbol{v}_{H} \in V_{H}^{s}}\left\|\partial_{t t} \boldsymbol{\eta}\left(t^{i}\right)-\boldsymbol{v}_{H}\right\|_{V^{s}}^{2}\right. \\
& \left.+\inf _{\boldsymbol{v}_{h} \in V_{h}^{f}}\left\|\partial_{t} \boldsymbol{u}\left(t^{i}\right)-\boldsymbol{v}_{h}\right\|_{V^{f}}^{2}+C h^{2 l}\left\|\partial_{t t} \boldsymbol{\eta}\left(t^{i}\right)\right\|_{V^{s}}^{2}\right) \\
\leq & C \delta t^{2}+C h^{2 k}+C H^{2 m}+C h^{2 l} .
\end{aligned}
$$

All the other terms of $\mathcal{H}$ can be bounded in a similar manner, consequently, using 76 :

$$
\mathcal{H} \leq C \delta t^{2}+C h^{2 k}+C H^{2 m}+C h^{2 l}+C \frac{h \rho^{f}}{H^{\alpha}} \delta t^{2} .
$$


Finally, the stability conditions $(19)$ imply:

$$
\mathcal{H} \leq C \delta t^{2}+C h^{2 k}+C H^{2 m}+C h^{2 l} .
$$

Let us now consider

$$
\begin{array}{r}
\mathcal{T} \stackrel{\text { def }}{=} \sum_{n=0}^{N} C\left(\frac{h \rho^{f}}{H^{\alpha}}+\frac{\mu \delta t}{h H^{\alpha}}\right)\left\|\frac{\boldsymbol{\eta}_{H}\left(t^{n+1}\right)-2 \boldsymbol{\eta}_{H}\left(t^{n}\right)+\boldsymbol{\eta}_{H}\left(t^{n-1}\right)}{\delta t}\right\|_{0, \Omega^{s}}^{2} \\
+\sum_{n=0}^{N} \frac{C \delta t^{2}}{\rho^{f}}\left\|\nabla p_{h}\left(t^{n+1}\right)\right\|_{0, \Omega^{f}}^{2} .
\end{array}
$$

Using (77), $\mathcal{T}$ can be bounded by

$$
\mathcal{T} \leq C\left(\frac{h \rho^{f}}{H^{\alpha}}+\frac{\mu \delta t}{h H^{\alpha}}+1\right) \delta t
$$

By applying the stability conditions $(19,, \mathcal{T}$ can be bounded by $C \delta t$, which leads to a time error estimate of order $\sqrt{\delta t}$.

Remark 8 Note that the reduction of the convergence rate in time is due to two different contributions. The first part, associated with the term $\left\|\frac{\boldsymbol{\eta}_{H}\left(t^{n+1}\right)-2 \boldsymbol{\eta}_{H}\left(t^{n}\right)+\boldsymbol{\eta}_{H}\left(t^{n-1}\right)}{\delta t}\right\|_{0, \Omega^{s}}^{2}$, comes intrinsically from the semi-implicit coupling (see Remark 5). The second part, associated with the term $\left\|\nabla p_{h}\left(t^{n+1}\right)\right\|_{0, \Omega^{f}}^{2}$, comes from the non-incremental Chorin-Temam scheme. It is well-known that this scheme has a reduced time accuracy in a pure hydrodynamic problem. Nevertheless, we must observe that, for the non-incremental Chorin-Temam scheme, the reduced time accuracy affects the fluid pressure in the $L^{\infty}\left(0, T ; L^{2}\left(\Omega^{f}\right)\right)$ norm and the fluid velocity error in the $L^{\infty}\left(0, T ; H^{1}\left(\Omega^{f}\right)\right)$ norm (see for example [33]). Here we also obtain a reduced time accuracy for the fluid velocity error in the $L^{\infty}\left(0, T ; L^{2}\left(\Omega^{f}\right)\right)$ norm. This may be due to the way we derived our error estimate as well as to the fact that the reduced time accuracy observed for the pressure and the fluid velocity affects the whole fluid-structure scheme.

Remark 9 Note finally that, if in (81) we don't apply the stability condition, in some particular case (e.g. $\alpha=0$ ) the convergence rate in time of the first term of $\mathcal{T}$ could be slightly better than $\delta t$. 
Consequently, from estimate $(74)$ and using $(75),(79)$ and $(81)$, we obtain

$$
\begin{aligned}
E^{N+1}+ & \frac{\rho^{f}}{4} \sum_{n=0}^{N}\left[\left\|\tilde{e}_{h}^{n+1}-e_{h}^{n}\right\|_{0, \Omega^{f}}^{2}+\left\|e_{h}^{n+1}-\tilde{e}_{h}^{n+1}\right\|_{0, \Omega^{f}}^{2}\right] \\
+ & \sum_{n=0}^{N} \mu \delta t\left\|\boldsymbol{\epsilon}\left(\tilde{e}_{h}^{n+1}\right)\right\|_{0, \Omega^{f}}^{2}+\frac{1}{2} \sum_{n=0}^{N} a^{s}\left(e_{H}^{n+1}-e_{H}^{n}, e_{H}^{n+1}-e_{H}^{n}\right) \\
+ & \sum_{n=0}^{N}\left(\frac{\rho^{s}}{2}-\frac{C h \rho^{f}}{H^{\alpha}}-\frac{C \mu \delta t}{h H^{\alpha}}\right)\left\|\frac{e_{H}^{n+1}-2 e_{H}^{n}+e_{H}^{n-1}}{\delta t}\right\|_{0, \Omega^{s}}^{2} \\
\leq & \sum_{n=0}^{N} C \rho^{f} \delta t\left(\left\|\tilde{e}_{h}^{n+1}-e_{h}^{n+1}\right\|_{0, \Omega^{f}}^{2}+\left\|e_{h}^{n+1}\right\|_{0, \Omega^{f}}^{2}\right) \\
& +\sum_{n=0}^{N} C \rho^{s} \delta t\left\|\frac{e_{H}^{n+1}-e_{H}^{n}}{\delta t}\right\|_{0, \Omega^{s}}^{2}+\sum_{n=0}^{N} \frac{C h \rho^{f} \delta t}{H^{\alpha}}\left\|\frac{e_{H}^{n+1}-2 e_{H}^{n}+e_{H}^{n-1}}{\delta t}\right\|_{0, \Omega^{s}}^{2} \\
& +\sum_{n=1}^{N} C \delta t a^{s}\left(e_{H}^{n}, e_{N}^{n}\right)+C \delta t+C h^{2 k}+H^{2 m}+C h^{2 l},
\end{aligned}
$$

where $\frac{1}{2} \leq l \leq k$ for the mortar case and $l \leq \min \left(r-\frac{\alpha}{2}, k+1\right)$ for the interpolation case. Note that we can restrict ourselves to case where $l \leq k$ for both matching operators since $r$ is typically equal to 1 or 2 .

The analysis is concluded applying a discrete version of the Gronwall's inequality. Here, for the sake of completeness, we recall only the result, referring to 32 for a proof of it.

Lemma 2 Let $\delta, g^{0}, a^{n}, b^{n}, c^{n}$ and $\gamma_{n}$ be a sequence of non negative numbers for integers $n \geq 0$ so that

$$
a^{n}+\delta \sum_{j=0}^{n} b^{j} \leq \delta \sum_{j=0}^{n} \gamma_{j} a^{j}+\delta \sum_{j=0}^{n} c^{j}+g^{0}
$$

Assume that $\gamma_{j} \delta<1$ for all $j$, and set $\sigma_{j}=\left(1-\gamma_{j} \delta\right)^{-1}$. Then, for all $n \geq 0$.

$$
a^{n}+\delta \sum_{j=0}^{n} b^{j} \leq \exp \left(\delta \sum_{j=0}^{n} \sigma_{j} \gamma_{j}\right)\left[\delta \sum_{j=0}^{n} c^{j}+g^{0}\right] .
$$

Assuming that the stability condition 190 holds true, the discrete Gronwall's lemma yields

$$
\begin{gathered}
E^{N+1}+\frac{\rho^{f}}{4} \sum_{n=0}^{N}\left[\left\|\tilde{e}_{h}^{n+1}-e_{h}^{n}\right\|_{0, \Omega^{f}}^{2}+\left\|e_{h}^{n+1}-\tilde{e}_{h}^{n+1}\right\|_{0, \Omega^{f}}^{2}\right]+\sum_{n=0}^{N} \mu \delta t\left\|\boldsymbol{\epsilon}\left(\tilde{e}_{h}^{n+1}\right)\right\|_{0, \Omega^{f}}^{2} \\
+\frac{1}{2} \sum_{n=0}^{N} a^{s}\left(e_{H}^{n+1}-e_{H}^{n}, e_{H}^{n+1}-e_{H}^{n}\right)+\sum_{n=0}^{N}\left(\frac{\rho^{s}}{2}-\frac{C h \rho^{f}}{H^{\alpha}}-\frac{C \mu \delta t}{h H^{\alpha}}\right)\left\|\frac{e_{H}^{n+1}-2 e_{H}^{n}+e_{H}^{n-1}}{\delta t}\right\|_{0, \Omega^{s}}^{2} \\
\leq C \delta t+C h^{2 k}+C H^{2 m}+C h^{2 l}, \quad(83)
\end{gathered}
$$

that concludes the derivation of error estimate 400. 


\section{$6 \quad$ Numerical results}

In this section we investigate numerically the order of convergence in time of the semi-implicit coupling scheme. Some computational results that confirm the previous analysis will be presented. Moreover, the numerical experiments realized give a deeper insight into the accuracy of the scheme that could be useful for further improvements of the theoretical analysis.

First the two-dimensional test case used for all the simulations will be introduced. Later, the convergence rate of the semi-implicit scheme 12- 13 . will be evaluated and compared to the one of other FSI algorithms. All the computation have been performed with FreeFem ++ [31].

The test case. A two-dimensional test case consisting of the following analytical solution over the domains $\Omega^{f}=[0,1] \times[0,1]$ and $\Omega^{s}=[0,1] \times[1,1.25]$ (Figure 2) is considered:

$$
\left\{\begin{aligned}
u_{x} & =\cos (x+t) \sin (y+t)+\sin (x+t) \cos (y+t), \\
u_{y} & =-\sin (x+t) \cos (y+t)-\cos (x+t) \sin (y+t), \\
p & =2 \mu(\sin (x+t) \sin (y+t)-\cos (x+t) \cos (y+t))+2 \Lambda_{2} \cos (x+t) \sin (y+t), \\
\eta_{x} & =\sin (x+t) \sin (y+t), \\
\eta_{y} & =\cos (x+t) \cos (y+t),
\end{aligned}\right.
$$

where $\Lambda_{2}$ stands for the second Lamé constant of solid. For the fluid, the physical parameters are $\rho_{\mathrm{f}}=1.0 \mathrm{~g} / \mathrm{cm}^{3}, \mu=0.013$ poise. For the solid, we have $\rho_{\mathrm{s}}=1.9 \mathrm{~g} / \mathrm{cm}^{3}$, the Lamé constant $\Lambda_{2}=3 \mathrm{dyne} / \mathrm{cm}^{2}$, and the Poisson's ratio $\nu=0.3$.

External boundary conditions, initial conditions and body forces, for the fluid and the structure, are chosen in order to ensure that the coupled system (2)-(3) is satisfied by the exact solution (84). In particular, considering Figure 2. Dirichlet and Neumann boundary conditions are respectively imposed on $\Gamma^{d}$ and $\Gamma^{n}$

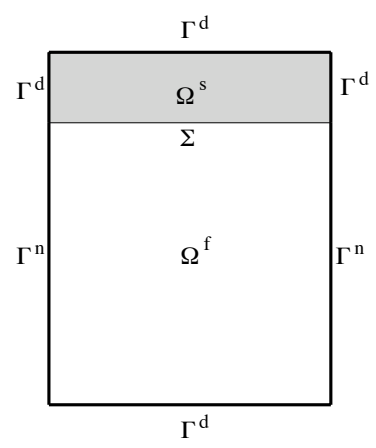

Figure 2: Computational domain.

For all the numerical simulations, we have adopted a conforming matching between the fluid and the structure without any consequence on the stability of the scheme (see Section 3). A uniform space discretization step $h=0.05 \mathrm{~cm}$ has 
been set. For the fluid, we have resorted Taylor-Hood finite element, while for the structure, $\mathbb{P}_{2}$ finite elements have been used in order to guarantee a small space discretization error. For the time discretization, a sequence of decreasing time steps $\left(\delta t=5 \cdot 10^{-2}, 2.5 \cdot 10^{-2}, 1.25 \cdot 10^{-2}, 6.25 \cdot 10^{-3}, 3.125 \cdot 10^{-3} \mathrm{~s}\right)$ has been used to compare the numerical solutions to the exact one (84).

Three different FSI algorithms are compared: the semi-implicit scheme (12)(13), a fully implicit scheme where the Stokes equations are solved as a mixed problem in the velocity and pressure unknowns, and a modified version of the semi-implicit scheme where we replace the non-incremental Chorin-Temam method with its incremental version (see for instance [29]). The latter is therefore given by:

Step 1: (explicit viscous-structure coupling)

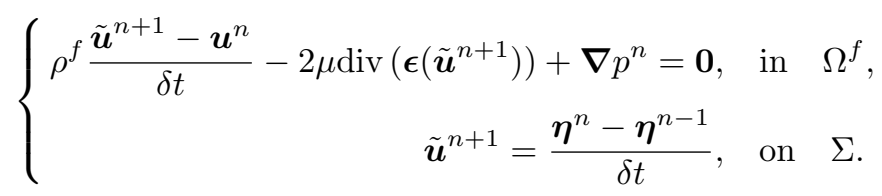

Step 2: (implicit pressure-structure coupling)

- Fluid projection sub-step:

$$
\left\{\begin{aligned}
\rho^{f} \frac{\boldsymbol{u}^{n+1}-\tilde{\boldsymbol{u}}^{n+1}}{\delta t}+\nabla\left(p^{n+1}-p^{n}\right)=\mathbf{0}, & \text { in } \quad \Omega^{f} \\
\operatorname{div} \boldsymbol{u}^{n+1}=0, & \text { in } \Omega^{f} \\
\boldsymbol{u}^{n+1} \cdot \mathbf{n}^{f}=\frac{\boldsymbol{\eta}^{n+1}-\boldsymbol{\eta}^{n}}{\delta t} \cdot \mathbf{n}^{f}, & \text { on } \quad \Sigma .
\end{aligned}\right.
$$

- Solid sub-step:

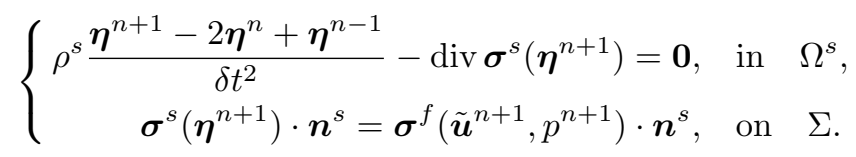

Moreover, for both of the semi-implicit schemes, the fluid part of the projection step has been rewritten as a Poisson problem for the pressure (see [1, 28, for more details). For the sake of conciseness, in the following, we address the previous schemes respectively as: non-incremental semi-implicit scheme, fully implicit scheme and incremental semi-implicit scheme.

The errors, between the numerical solutions and the exact one, are computed with respect to the norms $l^{\infty}\left(\tau, T, L^{2}\left(\Omega^{f}\right)\right)$, for $\boldsymbol{u}$ and $p, l^{\infty}\left(\tau, T, L^{2}\left(\Omega^{s}\right)\right)$, for the structure velocity $\boldsymbol{w} \stackrel{\text { def }}{=} \partial_{t} \boldsymbol{\eta}$, and $l^{\infty}\left(\tau, T, H^{1}\left(\Omega^{s}\right)\right)$, for $\boldsymbol{\eta}$, with $\tau=0.5$ and $T=1$. They are presented in Figure 3 and reported in logarithmic scale as a function of $\delta t$ : in red for the non-incremental semi-implicit algorithm, in green for the incremental one while in blue for the fully implicit scheme.

Non-incremental semi-implicit scheme versus fully implicit scheme. Let us first consider the time discretization error of the two algorithms. From Figure 3, it can be easily observed that for a given time step the discretization 
error is smaller in the fully implicit scheme than in the non-incremental semiimplicit scheme. As we will see later, this difference is mainly due to the use of the non-incremental Chorin-Temam scheme in the fluid problem.

For the convergence in time, as expected, the fully implicit scheme is first order accurate in time for velocity, pressure and displacement. Instead, for the semi-implicit one, we observe a lower convergence rate if compared to the implicit scheme. We can therefore confirm also from a numerical point of view that the scheme (12)-(13) has a convergence rate globally lower than one. In detail, it is important to note that a small reduction of convergence is experienced for the fluid pressure and for the structure unknowns, but not for the fluid velocity. The latter is indeed still linear in time. Therefore, at least in this test case, it seems that the velocity error with respect to the norm $l^{\infty}\left(\tau, T, L^{2}\left(\Omega^{f}\right)\right)$ isn't affected by a reduced convergence rate.

Remark 10 It is worth noticing that the test case proposed is only one of the many different analyzed and in none of them we have observed a reduction in the velocity accuracy in the $l^{\infty}\left(\tau, T, L^{2}\left(\Omega^{f}\right)\right)$ norm.

The convergence in time of the velocity unknowns remains therefore an open problem for the semi-implicit scheme. Possible improvements can be done either in the choice of the test case, or in a non-trivial improvement of our theoretical result, that aims at decoupling the analysis of the velocity error from the one of the pressure and displacement errors, in order to retrieve its linear convergence rate.

Non-incremental semi-implicit scheme versus incremental semi-implicit scheme. Here the convergence in time of the semi-implicit scheme is investigated by comparing the non-incremental version of the semi-implicit scheme with the incremental one. It is well-known that, for pure Stokes and NavierStokes problems, the incremental version of the Chorin-Temam scheme has better accuracy properties than the original one (see for example [18, 27, 28, 29] and references therein). A linear convergence in time is indeed retrieved for velocities and pressure (for a proof refer to [28, 41]):

$$
\begin{aligned}
& \left\|\boldsymbol{u}\left(t^{n}\right)-\boldsymbol{u}_{h}^{n}\right\|_{l^{\infty}\left(L^{2}\left(\Omega^{f}\right)\right)}+\left\|\boldsymbol{u}\left(t^{n}\right)-\widetilde{\boldsymbol{u}}_{h}^{n}\right\|_{l^{\infty}\left(L^{2}\left(\Omega^{f}\right)\right)} \leq c\left(h^{k+1}+\delta t\right), \\
& \left\|\boldsymbol{u}\left(t^{n}\right)-\widetilde{\boldsymbol{u}}_{h}^{n}\right\|_{l^{\infty}\left(H^{1}\left(\Omega^{f}\right)\right)}+\left\|p\left(t^{n}\right)-p_{h}^{n}\right\|_{l^{\infty}\left(L^{2}\left(\Omega^{f}\right)\right)} \leq c\left(h^{k}+\delta t\right) .
\end{aligned}
$$

A direct comparison between the numerical results of the two schemes, Figure 3. clearly shows that the choice of the numerical method for the fluid problem changes the accuracy in time of the whole fluid-structure interaction procedure. In detail, the non-incremental Chorin-Temam method induces a numerical dissipation on the coupled problem and modifies the convergence in time of the structure problem. With the incremental semi-implicit scheme a linear convergence rate in the fluid-structure problem seems to be retrieved.

Incremental semi-implicit scheme versus fully implicit scheme. Through this last comparison we stress once more the efficiency of the semi-implicit algorithm proposed in [20] and, in particular here, of its incremental version. Indeed, the latter is computationally cheaper than the fully implicit algorithm but it guarantees the same convergence order and similar discretization errors in time 
(see Figure 3). Finally we note that the semi-implicit coupling does not seem to affect the convergence of the incremental scheme. A deeper investigation of this last point will be addressed in future works both from a theoretical and numerical point of view.
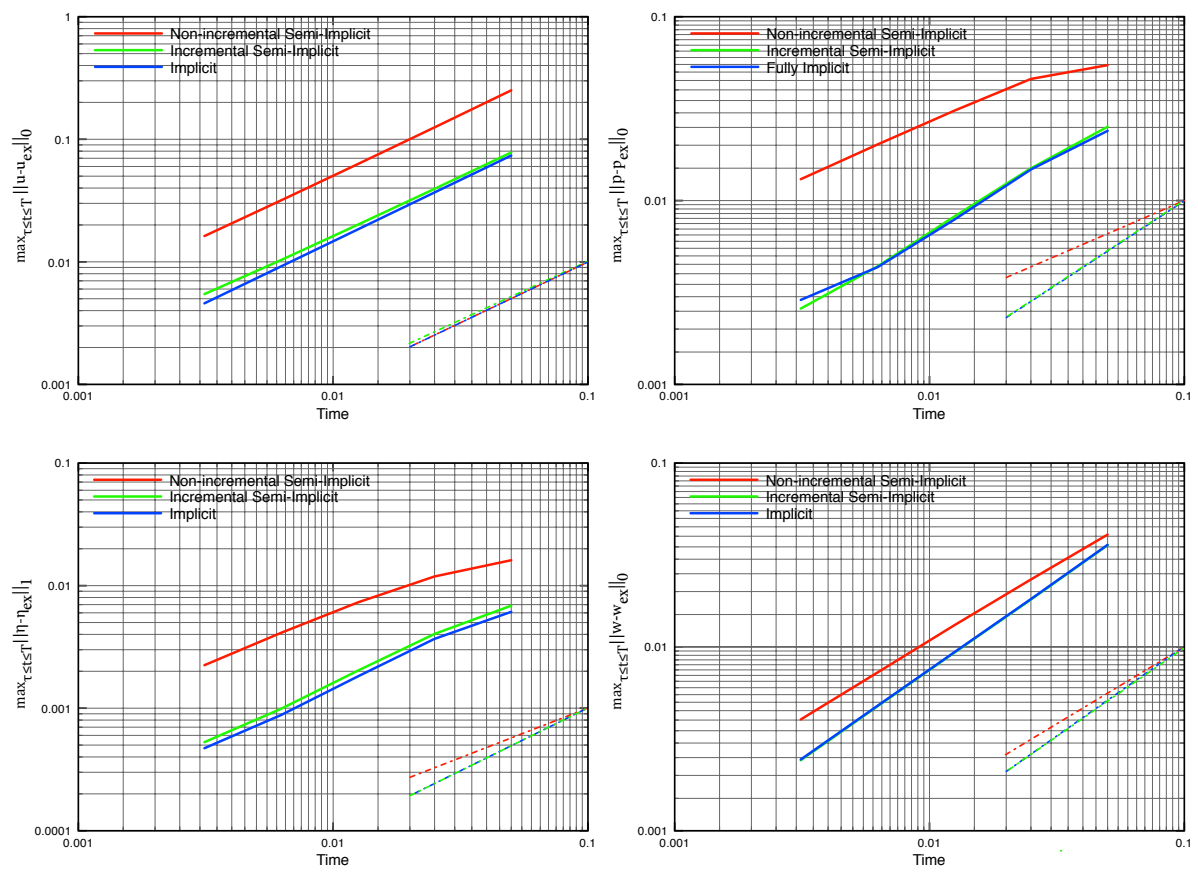

Figure 3: Convergence of the different FSI algorithms to the exact solution, for velocities, pressure and displacement. In red the non-incremental version of the semi-implicit scheme, in green the incremental one, while in blue the fully implicit scheme. Dashed lines are used to compare the slopes of the represented curves.

Before concluding, some more remarks are in order:

- The choice of the test case and of the physical and numerical parameters has been accurately set in order to satisfy the stability condition 19 and to stress the effects of a convergence rate lower than one in the nonincremental semi-implicit scheme. As a matter of fact, depending on the exact solution considered or on the parameters chosen, a linear convergence rate could be observed for some of (or even all) the unknowns.

- In the non-incremental semi-implicit scheme, we observe numerically a difference in the convergence rates of the fluid velocity and pressure. This difference seems to be in agreement with the estimates (1) and with the results reported in [27, 28, 29, 39] for the pure Stokes and Navier-Stokes problems. In addition for a pure fluid problem, numerical experiments done by other investigators (see [24, Chapter 7] ) show a substantial improvement in accuracy if in the Darcy formulation of the projection step one replaces the boundary condition on the normal component of the velocity by a full Dirichlet, which makes sense for the fully discrete problem. 
Clearly, this technique could be also used in the sub-problem (17) of the coupling algorithm.

\section{Conclusion}

In this paper we have analyzed from a theoretical and numerical point of view the semi-implicit scheme proposed in [20]. From a theoretical point of view the convergence of the scheme has been proved using a non-conforming space discretization of the two domains and the Leap-Frog scheme for the structure time discretization. The cases of mortar and interpolation matching operator have been considered. The study for the Newmark scheme is still an open question, even if, from a computational point of view, it is known to lead to stable algorithms.

We proved that the projection semi-implicit coupling scheme proposed in 20] is at least $\sqrt{\delta t}$ accurate in time. We supported our theoretical result with a numerical test where a small reduction of the convergence rate for pressure and displacement is observed. However, the effects of the coupling scheme on the fluid velocity remain an open question since no accuracy reductions have been experienced. Finally the use of the incremental version of the Chorin-Temam method in the fluid-structure interaction scheme actually improves the global accuracy and a linear convergence rate is retrieved for all the unknowns.

\section{Acknowledgements}

This work has been partially supported by the CardioSense3D action (INRIA).

\section{References}

[1] M. Astorino, F. Chouly, and F. Fernandez. An added-mass free semiimplicit coupling scheme for fluid-structure interaction. C. R. Acad. Sci. Paris, Sér. I, 347(1-2):99-104, 2009.

[2] S. Badia and R. Codina. Convergence analysis of the FEM approximation the first order Projection method for incompressible flows with and without the inf-sup condition. Numer. Math., 107(4):533-557, 2007.

[3] S. Badia, F. Nobile, and C. Vergara. Fluid-structure partitioned procedures based on Robin transmission conditions. J. Comp. Phys., 227:7027-7051, 2008.

[4] S. Badia, A. Quaini, and A. Quarteroni. Splitting methods based on algebraic factorization for fluid-structure interaction. SIAM J. Scientific Computing, 30(4):1778-1805, 2008.

[5] F. Ben Belgacem. The mortar finite element method with Lagrange multipliers. Numer. Math., 84(2):173-197, 1999.

[6] F. Ben Belgacem and Y. Maday. The mortar element method for three-dimensional finite elements. RAIRO Modél. Math. Anal. Numér., 31(2):289-302, 1997. 
[7] C. Bernardi, Y. Maday, and A. T. Patera. Domain decomposition by the mortar element method. In Asymptotic and numerical methods for partial differential equations with critical parameters (Beaune, 1992), volume 384 of NATO Adv. Sci. Inst. Ser. C Math. Phys. Sci., pages 269-286. Kluwer Acad. Publ., Dordrecht, 1993.

[8] C. Bernardi, Y. Maday, and A. T. Patera. A new nonconforming approach to domain decomposition: the mortar element method. In Nonlinear partial differential equations and their applications. Collège de France Seminar, Vol. XI (Paris, 1989-1991), volume 299 of Pitman Res. Notes Math. Ser., pages 13-51. Longman Sci. Tech., 1994.

[9] F. Brezzi. On the existence uniqueness and approximation of saddle-point problems arising from lagrangian multipliers. R.A.I.R.O.R., 2:129-151, 1974.

[10] F. Brezzi and M. Fortan. Mixed and hybrid finite element methods. Springer-Verlag, New York, 1991.

[11] E. Burman and M.A. Fernández. Stabilization of explicit coupling in fluidstructure interaction involving fluid incompressibility. Comput. Methods Appl. Mech. Engrg., 198(5-8):766-784, 2009.

[12] P. Causin, J.-F. Gerbeau, and F. Nobile. Added-mass effect in the design of partitioned algorithms for fluid-structure problems. Comp. Meth. Appl. Mech. Engng., 194(42-44):4506-4527, 2005.

[13] A.J. Chorin. Numerical solution of the Navier-Stokes equations. Math. Comp., 22:745-762, 1968.

[14] A.J. Chorin. On the convergence of discrete approximations to the NavierStokes equations. Math. Comp., 23:341-353, 1969.

[15] P.G. Ciarlet. Mathematical elasticity. Vol. I, volume 20 of Studies in Mathematics and its Applications. North-Holland Publishing Co., Amsterdam, 1988.

[16] P.G. Ciarlet and J.-L. Lions, editors. Handbook of Numerical Analysis, Vol. 2, Finite Element Methods (Part 1). North-Holland, 1991.

[17] Q. Du, M.D. Gunzburger, L.S. Hou, and J. Lee. Analysis of a linear fluidstructure interaction problem. Disc. Cont. Dyn. Sys, 9(3):633-650, 2003.

[18] A. Ern and J.-L. Guermond. Theory and practice of finite elements, volume 159 of Applied Mathematical Sciences. Springer-Verlag, New York, 2004.

[19] M. A. Fernández and M. Moubachir. Numerical simulation of fluidstructure systems via Newton's method with exact Jacobians. In P Neittaanmäki, T. Rossi, K. Majava, and O. Pironneau, editors, $4^{\text {th }}$ European Congress on Computational Methods in Applied Sciences and Engineering, volume 1, Jyväskylä, Finland, July 2004.

[20] M.A. Fernández, J.-F. Gerbeau, and C. Grandmont. A projection semiimplicit scheme for the coupling of an elastic structure with an incompressible fluid. Int. J. Numer. Meth. Engng, 69(4):794-821, 2007. 
[21] C. Foerster, W. A. Wall, and E. Ramm. The artificial added mass effect in sequential staggered fluid-structure interaction algorithms. In P. Wessling, E. Onate, and J. Periaux, editors, Prooceedings European Conference on Computational Fluid Dynamics ECCOMAS CFD, 2006.

[22] L. Formaggia, A. Quarteroni, and A. Veneziani, editors. Cardiovascular Mathematics. Modeling and simulation of the circulatory system, volume 1 of Modeling, Simulation and Applications. Springer, 2009.

[23] J.-F. Gerbeau and M. Vidrascu. A quasi-Newton algorithm based on a reduced model for fluid-structure interactions problems in blood flows. Math. Model. Num. Anal., 37(4):631-648, 2003.

[24] R. Glowinski. Finite element methods for incompressible viscous flow, volume IX of Handbook of Numerical Analysis. North-Holland, Amsterdam, 2003.

[25] C. Grandmont. Analyse mathématiques et numérique de quelques problèmes d'interaction fluid-structure. PhD thesis, Université Pierre et Marie Curie - Paris 6, 1998.

[26] C. Grandmont and Y. Maday. Existence for an unsteady fluid-structure interaction problem. M2AN Math. Model. Numer. Anal., 34(3):609-636, 2000 .

[27] J.-L. Guermond, P. Minev, and J. Shen. An overview of projection methods for incompressible flows. Comput. Methods Appl. Mech. Engng., 195(4447):6011-6045, 2006.

[28] J.-L. Guermond and L. Quartapelle. On stability and convergence of projection methods based on pressure Poisson equation. Internat. J. Numer. Methods Fluids, 26(9):1039-1053, 1998.

[29] J.-L. Guermond and L. Quartapelle. On the approximation of the unsteady Navier-Stokes equation by finite element projection methods. $\mathrm{Nu}$ mer. Math., 80(2):207-238, 1998.

[30] G. Guidoboni, R. Glowinski, N. Cavallini, and S. Canic. Stable looselycoupled-type algorithm for fluid-structure interaction in blood-flow. $J$. Comp. Phys., 228(18):6916-6937, 2009.

[31] F. Hecht, O. Pironneau, A. Le Hyaric, and K. Ohtsuka. FreeFem $++v$. 2.11. User's Manual. University of Paris 6.

[32] J.G. Heywood and R. Rannacher. Finite-element approximations of the nonstationary Navier-Stokes problem. Part IV: error estimates for secondorder time discretization. SIAM J. Numer. Anal., 27(2):353-384, 1990.

[33] Ulrich Hornung, editor. Projection and Quasi-compressibility Methods for Solving the Incompressible Navier-Stokes Equations. Springer-Verlag, New York, 1997.

[34] P. Le Tallec and S. Mani. Numerical analysis of a linearised fluid-structure interaction problem. Numer. Math., 87(2):317-354, 2000. 
[35] J.-L. Lions. Quelques méthodes de résolution des problèmes aux limites non linéaires. Dunod, 1969.

[36] D. P. Mok, W. A. Wall, and E. Ramm. Accelerated iterative substructuring schemes for instationary fluid-structure interaction. In K.J. Bathe, editor, Computational Fluid and Solid Mechanics, pages 1325-1328. Elsevier, 2001.

[37] A. Quaini and A. Quarteroni. A semi-implicit approach for fluid-structure interaction based on an algebraic fractional step method. Math. Models Methods Appl. Sci., 17(6):957-983, 2007.

[38] A. Quarteroni, F. Saleri, and A. Veneziani. Factorization methods for the numerical approximation of the Navier-Stokes equations. Comp. Methods in Appl. Mech. Engng., 188(1-3):505-526, 2000.

[39] R. Rannacher. On Chorin's projection method for the incompressible Navier-Stokes equations. Lecture Notes in Mathematics, 1530:167-183, 1991.

[40] F. Saleri and A. Veneziani. Pressure correction algebraic-splitting methods for the incompressible Navier-Stokes equations. SIAM J. Numer. Anal., 43(1):174-194, 1999.

[41] J. Shen. On error estimates of projection methods for the navier-stokes equations: First-order schemes. SIAM J. Numeri. Anal., 29:55-77, 1992.

[42] T. Takahashi. Analyse des équations modélisant le mouvement des systémes couplant des solides rigides et des fluides visqueux. $\mathrm{PhD}$ thesis, Université Henri Poincaré - Nancy I, 2002.

[43] R. Temam. Une méthode d'approximation de la solution des équations de Navier-Stokes. Bull. Soc. Math. France, 96:115-152, 1968.

\section{Contents}

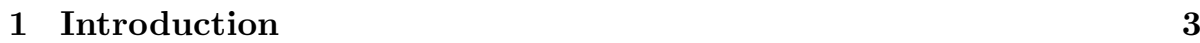

2 Problem setting 4

2.1 Hypotheses and notations . . . . . . . . . . . . . . . . . . 4

2.2 Variational formulation . . . . . . . . . . . . . . . 6

$\begin{array}{llr}3 & \text { Semi-implicit projection scheme } & 8\end{array}$

3.1 Fully discretized scheme . . . . . . . . . . . . . . . . . . . . . 10

3.2 Interface matching operators . . . . . . . . . . . . . . . . . . . . . 12

3.2.1 Pointwise matching . . . . . . . . . . . . . . . . . . . . . 12

3.2.2 Mortar matching . . . . . . . . . . . . . . . . . 12

4 Construction of the finite element approximations 13

$\begin{array}{lll}5 & \text { The error analysis } & 18\end{array}$

5.1 Main result . . . . . . . . . . . . . . . . . . . . . 19

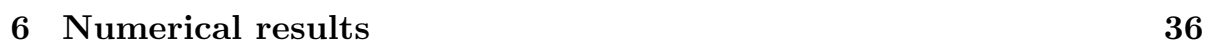

RR $\mathrm{n}^{\circ} 6996$ 
\begin{tabular}{lll}
\hline 7 Conclusion & 40
\end{tabular} 
Centre de recherche INRIA Paris - Rocquencourt Domaine de Voluceau - Rocquencourt - BP 105 - 78153 Le Chesnay Cedex (France)

Centre de recherche INRIA Bordeaux - Sud Ouest : Domaine Universitaire - 351, cours de la Libération - 33405 Talence Cedex Centre de recherche INRIA Grenoble - Rhône-Alpes : 655, avenue de l'Europe - 38334 Montbonnot Saint-Ismier Centre de recherche INRIA Lille - Nord Europe : Parc Scientifique de la Haute Borne - 40, avenue Halley - 59650 Villeneuve d'Ascq Centre de recherche INRIA Nancy - Grand Est : LORIA, Technopôle de Nancy-Brabois - Campus scientifique 615, rue du Jardin Botanique - BP 101 - 54602 Villers-lès-Nancy Cedex

Centre de recherche INRIA Rennes - Bretagne Atlantique : IRISA, Campus universitaire de Beaulieu - 35042 Rennes Cedex Centre de recherche INRIA Saclay - Île-de-France : Parc Orsay Université - ZAC des Vignes : 4, rue Jacques Monod - 91893 Orsay Cedex Centre de recherche INRIA Sophia Antipolis - Méditerranée : 2004, route des Lucioles - BP 93 - 06902 Sophia Antipolis Cedex 